Supporting Information

\title{
Chapter Open for Excited-State Intramolecular Thiol Proton Transfer in the Room-Temperature Solution
}

\author{
Chun-Hsiang Wang, ${ }^{\dagger, "}$ Zong-Ying Liu, ${ }^{\dagger, "}$ Chun-Hao Huang,, , Chao-Tsen Chen, ${ }^{\dagger *}$ \\ Fan-Yi Meng, ${ }^{\dagger}$ Yu-Chan Liao, ${ }^{\dagger}$ Yi-Hung Liu,${ }^{\dagger}$ Chao-Che Chang, ${ }^{\dagger}$ Elise Y. Li, ${ }^{\S *}$ Pi-Tai \\ $\mathrm{Chou}^{{ }^{*}}$ \\ ${ }^{\dagger}$ Department of Chemistry, National Taiwan University, Taipei, 10617, Taiwan, R.O.C. \\ ${ }^{\S}$ Department of Chemistry, National Taiwan Normal University, Taipei, 11677, Taiwan, R.O.C. \\ "These three authors contributed equally \\ Corresponding Authors: \\ * Chao-Tsen Chen: chenct@ntu.edu.tw \\ * Elise Y. Li: eliseytli@ntnu.edu.tw \\ * Pi-Tai Chou: chop@ntu.edu.tw
}




\section{Table of Content}

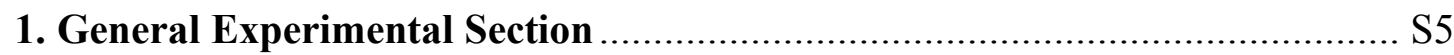

2. Experimental Procedures......................................................................... S5

Figure S1. ${ }^{1} \mathrm{H}-\mathrm{NMR}$ spectrum in $\mathrm{CDCl}_{3}$ acquired from route 1 in scheme 1 indicating the presence of $\mathbf{3 T F}$ and a small amount of SSF. The peaks highlighted in green colour corresponded to SSF.

Figure S2. The crude spectra (a) and (b) in $\mathrm{CDCl}_{3}$ acquired from step $\mathrm{h}$ of the route 2 in scheme 1 after acidified with $\mathrm{HCl}$ or citric acid, respectively, indicated the presence of $\mathbf{3 T F}$ as the major product accompanied with SSF as the minor product. The peaks highlighted in green colour corresponded to SSF. S16

Figure S3. The crude spectrum in $\mathrm{CDCl}_{3}$ acquired from step $\mathrm{b}$ of the route 1 in scheme 1 indicated only 3 TF present and no SSF evidenced after acidified with citric acid.

Figure S4. The sharp singlet peaks corresponding to the thiol protons of $\mathbf{3 T F}$,

3FTF, and 3NTF were evidenced in ${ }^{1} \mathrm{H}-\mathrm{NMR}$ S18

3. Structure Characterization S19

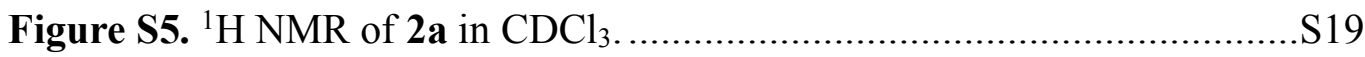

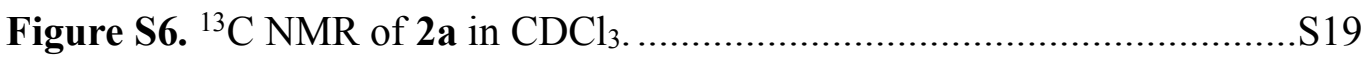

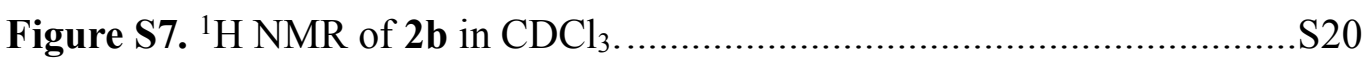

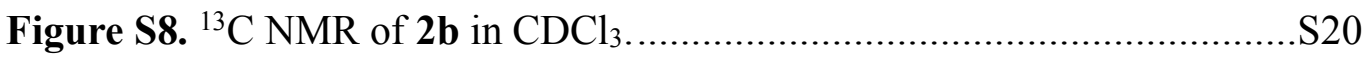

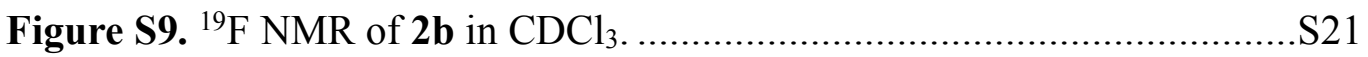

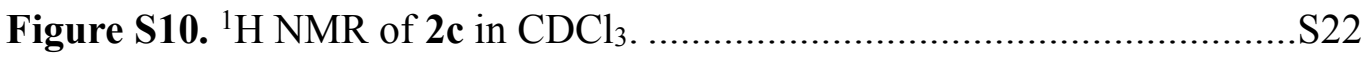

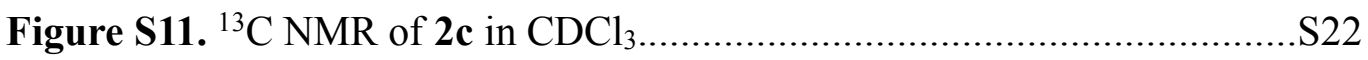

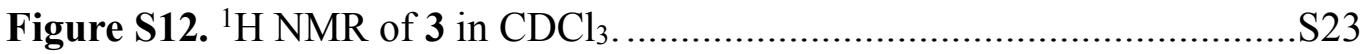

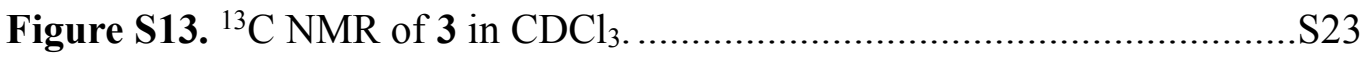

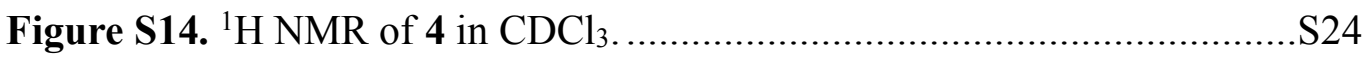

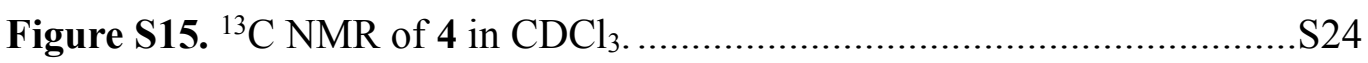

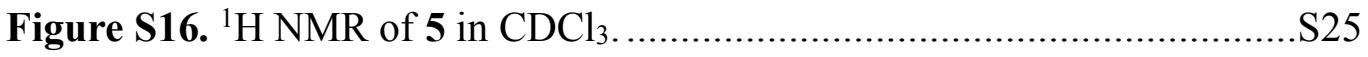

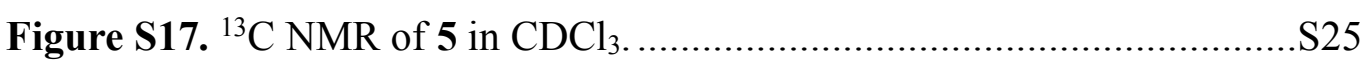

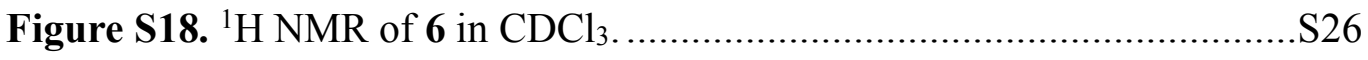

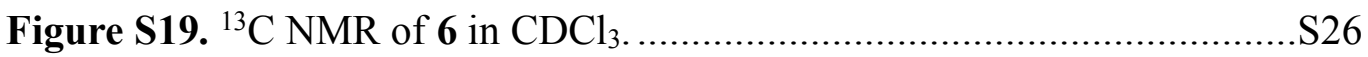

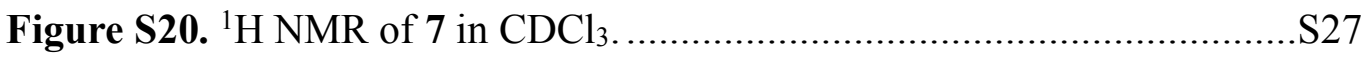

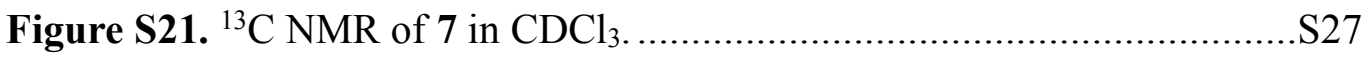

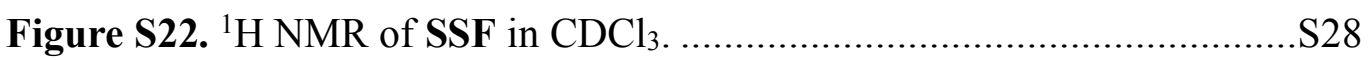

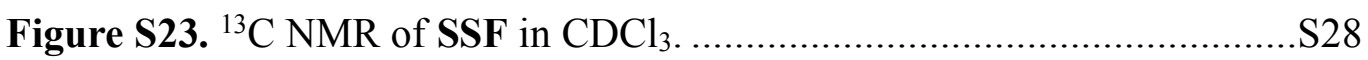

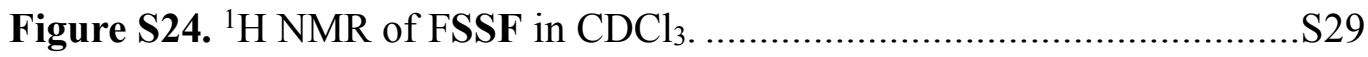

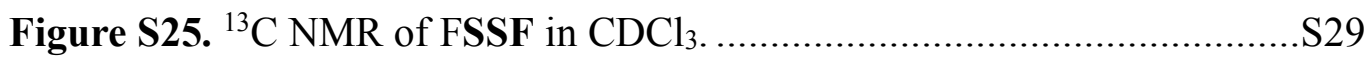




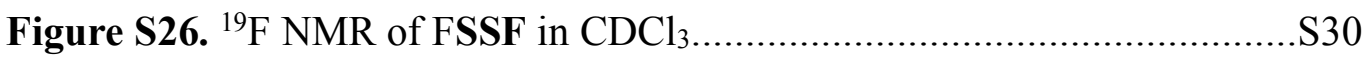

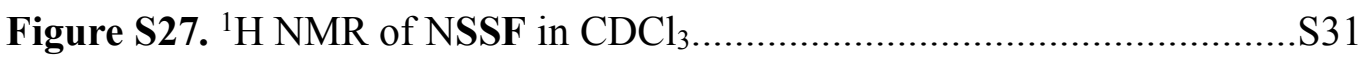

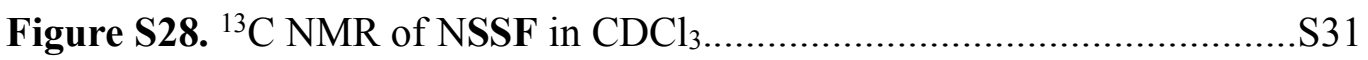

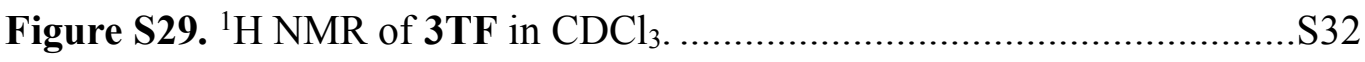

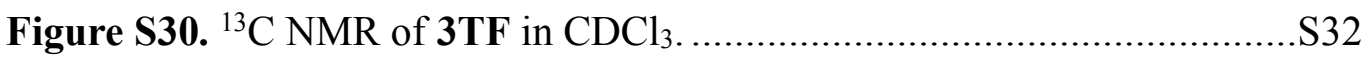

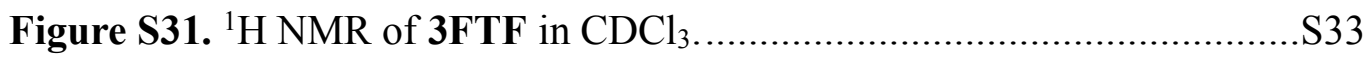

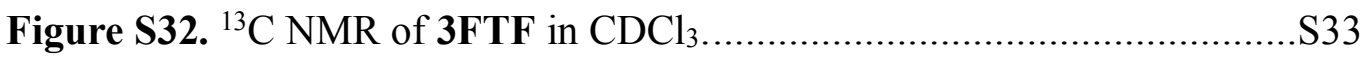

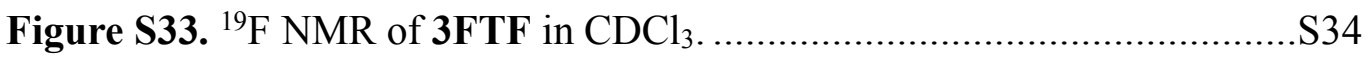

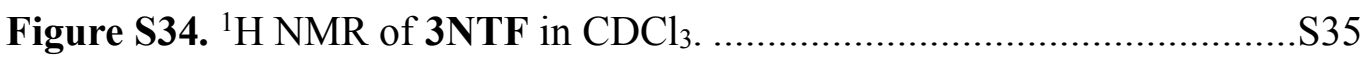

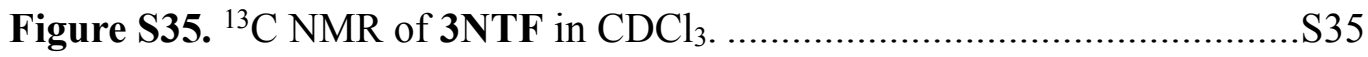

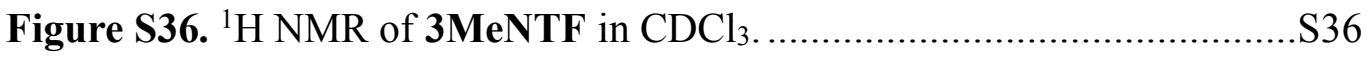

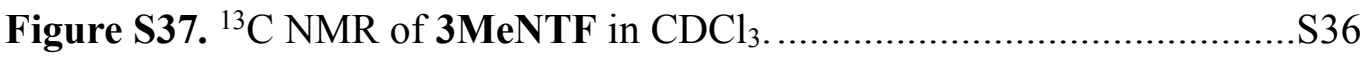

Figure S38. The mid-IR spectra of 3TF (top) and 3FTF (bottom)...................S37

Figure S39. The X-ray crystallographic structure of 3TF............................S37

Figure S40. The X-ray crystallographic structure of 3FTF...........................S37

Figure S41. The computed ground state geometries for (a) 3NTF, (b) 3TF and

(c) 3FTF at the B3LYP/6-311++G(3df,3pd) level.......................................S38

4. Photophysical Properties...........................................................................S39

Figure S42. (a) The absorption spectra of 3NTF in cyclohexane at different concentrations. (b) The emission spectra of $\mathbf{3 N T F}$ in cyclohexane at different concentrations.

Figure S43. The transient absorption spectrum for 3NTF in cyclohexane at 298 $\mathrm{K}$.

Figure S44. The kinetic traces for the transient absorption signal at (a) $535 \mathrm{~nm}$ and (b) $710 \mathrm{~nm}$ in cyclohexane at $298 \mathrm{~K}$.

Table S1. Calculated excitation energies of the low-lying excited states in the optimized $\mathbf{D}_{\mathbf{0}}$ geometry of deprotonated $\mathbf{3 N T F}$ in the neutral radical form.

Figure S45. Calculated molecular orbitals in the optimized $\mathrm{D}_{0}$ geometry of deprotonated 3NTF in the neutral radical form at the UB3LYP/6$311++\mathrm{G}(3 \mathrm{df}, 3 \mathrm{pd})$ level.

Figure S46. The absorption (circle and line) and emission (solid line) spectra for 3NTF in ethanol at $298 \mathrm{~K}$.

Figure S47. The kinetic trace of 3NTF at (a) $640 \mathrm{~nm}$ and (b) $670 \mathrm{~nm}$ acquired by fluorescence up-conversion technique in cyclohexane at $298 \mathrm{~K}$.

Table S2. The time-resolved data for 3NTF acquired by fluorescence upconversion measurement in cyclohexane at $298 \mathrm{~K}$.

Figure S48. The calculated geometries for $3 \mathrm{NTF}$ at $\mathrm{S}_{1}$ and $\mathrm{S}_{1}$ ' states. The angle represents the dihedral angle between phenyl group and chromenone moiety..S44 
Figure S49. (a) The absorption (circle and line) and emission (solid line) spectra of $\mathbf{3 T F}$ in cyclohexane at $298 \mathrm{~K}$. The excitation spectrum of $\mathbf{3 T F}$ monitored at $540 \mathrm{~nm}$ is shown in red line. (b) The absorption (circle and line) and emission (solid line) spectra of 3FTF in cyclohexane at 298K.

Figure S50. The emission spectra of 3FTF in cyclohexane and cyclohexane + $\mathrm{HCl}$ gas at $298 \mathrm{~K}$.

Figure S51. the absorption (circle and line) and emission (black solid line) spectra of 3NTF in cyclohexane at 298K.

Table S3. Calculated excitation energies of the low-lying excited states in the optimized $\mathbf{S}_{1}$ geometry of deprotonated $\mathbf{3 N T F}$ with the $\mathrm{Na}^{+}$counterion as the ionpair form.

Figure S52. Calculated molecular orbitals in the optimized $\mathrm{S}_{1}$ geometry of deprotonated $\mathbf{3 N T F}$ with the $\mathrm{Na}^{+}$counterion as the ion-pair form at the B3LYP/6-311++G(3df,3pd) level.

Table S4. Calculated excitation energies of the low-lying excited states in the optimized $\mathbf{S}_{\mathbf{1}}$ geometry of deprotonated $\mathbf{3 N T F}$ in the anion form.

Figure S53. Calculated molecular orbitals in the optimized $\mathrm{S}_{1}$ geometry of deprotonated $\mathbf{3 N T F}$ in the anion form at the B3LYP/6-311++G(3df,3pd) level.

Figure S54. The calculated various energy levels for $\mathbf{3 N T F}$ in the optimized $\mathrm{S}_{0}$, $\mathrm{S}_{1}$ and $\mathrm{T}_{1}$ geometries at the normal form and the proton-transfer tautomer form (for which the states are denoted with a prime).

Figure S55. The calculated various energy levels for $\mathbf{3 T F}$ in the optimized $\mathrm{S}_{0}, \mathrm{~S}_{1}$ and $\mathrm{T}_{1}$ geometries at the normal form and the proton-transfer tautomer form (for which the states are denoted with a prime). S48

Figure S56. The calculated various energy levels for 3FTF in the optimized $\mathrm{S}_{0}$, $\mathrm{S}_{1}$ and $\mathrm{T}_{1}$ geometries at the normal form and the proton-transfer tautomer form (for which the states are denoted with a prime).

Figure S57. Calculated molecular orbitals in the optimized ground-state geometry of the normal or the tautomer forms (denoted by prime) of various compounds at the B3LYP/6-311++G(3df,3pd) level.

Table S5. Theoretical absorption and luminescence data for different systems in the normal and tautomer (denoted by prime) forms in cyclohexane.

Table S6. Calculated excitation energies of the low-lying excited states in the optimized $\mathbf{S}_{1}{ }_{1}$ geometry of $\mathbf{3 N T F}$ (in the tautomer form).

Table S7. Calculated excitation energies and the corresponding molecular orbital transitions of the low-lying excited states in the optimized $\mathbf{S}_{1}{ }_{1}$ geometry of $\mathbf{3 T F}$ 
(in the tautomer form)...

S50

Table S8. Calculated excitation energies of the low-lying excited states in the optimized $\mathbf{S}{ }_{1}$ geometry of $\mathbf{3 F T F}$ (in the tautomer form) S51 


\section{General Experimental Section}

All chemicals were purchased from commercial sources and used as received. Solvents used for syntheses were dried either by standard literature methods before being distilled or by the drying solvent system (SPBT-1 bench top solvent purification system, LC Technology Solutions, Inc) and stored under nitrogen over $4 \AA$ molecular sieves after drying. Merck silica gel 60 F254 was used as TLC plate, visualized by UV light or staining reagents such as PMA. Flash column chromatography was performed on Silica gel 60 (230-400 mesh ASTM). ${ }^{1} \mathrm{H}(400 \mathrm{MHz}),{ }^{13} \mathrm{C}(100 \mathrm{MHz})$, and ${ }^{19} \mathrm{~F}(376 \mathrm{MHz})$ spectra were recorded on Bruker AVIII 400 spectrometer. Chemical shifts are reported relative to TMS $(0 \mathrm{ppm})$ or $\mathrm{CDCl}_{3}(7.24 \mathrm{ppm})$ and quoted as $\delta$ values in ppm. The following abbreviations are used: singlet $(\mathrm{s})$, doublet $(\mathrm{d})$, triplet $(\mathrm{t})$ and multiplet $(\mathrm{m})$. Melting points were determined on a Fargo MP-1D melting point apparatus without further correction. Infrared spectra were recorded on Varian 640-IR spectrometer. Mass spectra with an electrospray ionization (ESI) were determined on a Bruker microTOFQ II spectrometer. Single crystal structures were determined by Rigaku Oxford Diffraction diffractometer (Xcalibur, Atlas, Gemini) with monochromator Mo-Karadiation $(\lambda=0.71073 \AA)$.

\section{Experimental Procedures}<smiles>O=c1cc(-c2ccccc2)oc2ccccc12</smiles>

2a

\section{2-phenyl-4H-chromen-4-one (2a) ${ }^{1}$}

To a solution of 2'-hydroxyacetophenone $(0.12 \mathrm{~mL}, 1 \mathrm{mmol})$ in acetone $(20 \mathrm{~mL})$ were added potassium carbonate $(1.11 \mathrm{~g}, 8 \mathrm{mmol}, 8.0$ equiv. $)$ and pyridine $(0.32 \mathrm{~mL}, 4 \mathrm{mmol}$, 4.0 equiv.). The resulting solution was stirred at reflux temperature for $10 \mathrm{~min}$, followed by the addition of benzoyl chloride $(0.23 \mathrm{~mL}, 2 \mathrm{mmol}, 2.0$ equiv.) within $10 \mathrm{~min}$. The solution was stirred and refluxed for additional $16 \mathrm{~h}$ and was filtered over celite afterwards. The filtrate was added $\mathrm{AcOH}(5 \mathrm{~mL})$, followed by refluxed for $24 \mathrm{~h}$. The resulting solution was concentrated, and water $(30 \mathrm{~mL})$ was added to the residue, followed by extraction with EtOAc $(30 \mathrm{~mL}$ x 3). The combined organic layers were washed with brine $(100 \mathrm{~mL})$, dried over anhydrous $\mathrm{MgSO}_{4}$, and concentrated. The crude product was purified by flash column chromatography $($ EtOAc/hexane $=1: 3)$ and recrystallized from acetone to give a white needle solid $\mathbf{2 a}(171 \mathrm{mg}, 77 \%)$. 
m.p. 101-102 ${ }^{\circ} \mathrm{C} ; R_{f}=0.29\left(\right.$ EtOAc/hexane, 1:3). ${ }^{1} \mathrm{H}-\mathrm{NMR}\left(\mathrm{CDCl}_{3}, 400 \mathrm{MHz}\right) \delta 8.22$ $(\mathrm{d}, J=7.9 \mathrm{~Hz}, 1 \mathrm{H}), 7.90(\mathrm{~d}, J=7.8 \mathrm{~Hz}, 2 \mathrm{H}), 7.68(\mathrm{t}, J=8.1 \mathrm{~Hz}, 1 \mathrm{H}), 7.60-7.50(\mathrm{~m}, 4 \mathrm{H})$, $7.40(\mathrm{t}, J=7.5 \mathrm{~Hz}, 1 \mathrm{H}), 6.83(\mathrm{~s}, 1 \mathrm{H}) .{ }^{13} \mathrm{C}-\mathrm{NMR}\left(\mathrm{CDCl}_{3}, 100 \mathrm{MHz}\right) \delta 178.4,163.4$, 156.3, 133.8, 131.8, 131.6, 129.0, 126.3, 125.2, 124.0, 118.1, 107.6. IR (ZnSe) 1645, $1606 \mathrm{~cm}^{-1}$. ESI-HRMS calcd for $\mathrm{C}_{15} \mathrm{H}_{11} \mathrm{O}_{2}\left(\mathrm{M}^{+}+1\right) 223.0754$, found 223.0765.<smiles>O=c1cc(-c2ccc(C(F)(F)F)cc2)oc2ccccc12</smiles>

2b

\section{2-(4-(trifluoromethyl)phenyl)-4H-chromen-4-one (2b)}

To the solution of 2'-hydroxyacetophenone (1.2 mL, $10 \mathrm{mmol})$ in EtOH (40 mL) was added $\mathrm{KOH}_{(\mathrm{aq})}(16.8 \mathrm{~g}, 300 \mathrm{mmol}$ in $36 \mathrm{~mL}$ of water) dropwise within $10 \mathrm{~min}$ at room temperature and then heated up to $50 \quad{ }^{\circ} \mathrm{C}$. Subsequently, 4(trifluoromethyl)benzaldehyde (1.6 mL, $12 \mathrm{mmol}, 1.2$ equiv.) was added and stirred at $50{ }^{\circ} \mathrm{C}$ for additional $3 \mathrm{~h}$. The reaction was then poured into ice-cooled water $(50 \mathrm{~mL})$, followed by addition of $10 \% \mathrm{HCl}$ to adjust the solution to $\mathrm{pH}=2$. The crude precipitate was collected and washed with water $(200 \mathrm{~mL})$, followed by dried in vacuo. To the solution of crude precipitate dissolving in DMSO $(20 \mathrm{~mL})$ was added iodine (13 $\mathrm{mg}$, $0.1 \mathrm{mmol}, 0.01$ equiv.) and heated up to $130{ }^{\circ} \mathrm{C}$ for $1 \mathrm{~h}$. The reaction was quenched with sat. $\mathrm{Na}_{2} \mathrm{~S}_{2} \mathrm{O}_{3(\text { aq })}(40 \mathrm{~mL})$ and extracted with $\mathrm{CHCl}_{3}(50 \mathrm{~mL}$ x 3). The combined organic layers were washed with brine $\left(100 \mathrm{~mL}\right.$ x 3), dried over anhydrous $\mathrm{MgSO}_{4}$, and concentrated. The crude residue was purified by flash column chromatography (EtOAc/hexane $=1: 4)$ and recrystallized from $\mathrm{CHCl}_{3} /$ hexane to give a white needle solid $2 \mathbf{b}(2.89 \mathrm{~g}, 56 \%)$.

m.p. $149-150{ }^{\circ} \mathrm{C} ; R_{f}=0.38($ EtOAc/hexane, $1: 3) .{ }^{1} \mathrm{H}-\mathrm{NMR}\left(\mathrm{CDCl}_{3}, 400 \mathrm{MHz}\right) \delta 8.23$ $(\mathrm{d}, J=7.9 \mathrm{~Hz}, 1 \mathrm{H}), 8.04(\mathrm{~d}, J=8.2 \mathrm{~Hz}, 2 \mathrm{H}), 7.78(\mathrm{~d}, J=8.3 \mathrm{~Hz}, 2 \mathrm{H}), 7.72(\mathrm{t}, J=7.7$ $\mathrm{Hz}, 1 \mathrm{H}), 7.58$ (d, $J=8.4 \mathrm{~Hz}, 1 \mathrm{H}), 7.43$ (t, $J=7.5 \mathrm{~Hz}, 1 \mathrm{H}), 6.86(\mathrm{~s}, 1 \mathrm{H}) .{ }^{13} \mathrm{C}-\mathrm{NMR}$ $\left(\mathrm{CDCl}_{3}, 100 \mathrm{MHz}\right) \delta 178,2,161.6,156.1,135.1,134.1,133.1$ (q, $\left.J=27.6 \mathrm{~Hz},{ }^{2} \mathrm{~J}_{\mathrm{CF}}\right)$, $130.4,126.6,126.0\left(\mathrm{q}, J=3.9 \mathrm{~Hz},{ }^{3} \mathrm{~J}_{\mathrm{CF}}\right), 125.7,125.5,124.6\left(\mathrm{q}, J=160 \mathrm{~Hz},{ }^{1} \mathrm{~J}_{\mathrm{CF}}\right), 118.0$, 108.6. ${ }^{19} \mathrm{~F}-\mathrm{NMR}\left(\mathrm{CDCl}_{3}, 376 \mathrm{MHz}\right) \delta$-63.0. IR (ZnSe) 1643, 1572, $1324 \mathrm{~cm}^{-1}$. ESIHRMS calcd for $\mathrm{C}_{16} \mathrm{H}_{10} \mathrm{~F}_{3} \mathrm{O}_{2}\left(\mathrm{M}^{+}+1\right) 291.0627$, found 291.0602 . 
<smiles>CN(C)c1ccc(-c2cc(=O)c3ccccc3o2)cc1</smiles>

2c

\section{2-(4-(diethylamino)phenyl)-4H-chromen-4-one (2c)}

Similar procedures used to prepare $\mathbf{2 b}$ was adopted to prepare $\mathbf{2 c}$. The crude residue was purified by flash column chromatography $(1.5 \% \mathrm{MeOH} / \mathrm{DCM})$ and recrystallized from $\mathrm{CHCl}_{3} /$ hexane to give an orange solid $2 \mathrm{c}(2.73 \mathrm{~g}, 37 \%)$.

m.p. $140-141{ }^{\circ} \mathrm{C} ; R_{f}=0.3(1.5 \% \mathrm{MeOH} / \mathrm{DCM}) .{ }^{1} \mathrm{H}-\mathrm{NMR}\left(\mathrm{CDCl}_{3}, 400 \mathrm{MHz}\right) \delta 8.19(\mathrm{~d}$, $J=7.5 \mathrm{~Hz}, 1 \mathrm{H}), 7.78(\mathrm{~d}, J=9.0 \mathrm{~Hz}, 2 \mathrm{H}), 7.63(\mathrm{~d}, J=8.2 \mathrm{~Hz}, 1 \mathrm{H}), 7.50(\mathrm{~d}, J=8.3 \mathrm{~Hz}$, $1 \mathrm{H}), 7.36(\mathrm{t}, J=7.5 \mathrm{~Hz}, 1 \mathrm{H}), 6.71(\mathrm{~d}, J=9.0 \mathrm{~Hz}, 2 \mathrm{H}), 6.67(\mathrm{~s}, 1 \mathrm{H}), 3.42(\mathrm{q}, J=8.0 \mathrm{~Hz}$, $4 \mathrm{H}), 1.20(\mathrm{t}, J=8.0 \mathrm{~Hz}, 6 \mathrm{H}) .{ }^{13} \mathrm{C}-\mathrm{NMR}\left(\mathrm{CDCl}_{3}, 100 \mathrm{MHz}\right) \delta 178,3,164.4,156.1,150.2$, 133.1, 128.0, 125.5, 124.7, 124.1, 117.8, 117.3, 111.1, 104.0, 44.5, 12.5. IR (ZnSe) 1736, $1599 \mathrm{~cm}^{-1}$. ESI-HRMS calcd for $\mathrm{C}_{19} \mathrm{H}_{20} \mathrm{NO}_{2}\left(\mathrm{M}^{+}+1\right)$ 294.1489, found 294.1458.<smiles>O=C(/C=C/c1ccccc1)c1ccccc1O</smiles>

\section{(E)-1-(2-hydroxyphenyl)-3-phenylprop-2-en-1-one (3)}

2'-Hydroxyacetophenone (1.36 g, $10 \mathrm{mmol})$, benzaldehyde (1.27 g, $12 \mathrm{mmol}, 1.2$ equiv.), and $\mathrm{EtOH}(20 \mathrm{~mL})$ were placed in a round bottom flask and stirred at $0{ }^{\circ} \mathrm{C}$ for 10 min under Ar. A solution containing $\mathrm{KOH}$ (10 g, 178 mmol, 17.8 equiv.), $\mathrm{H}_{2} \mathrm{O}$ (8 $\mathrm{mL})$, and $\mathrm{EtOH}(10 \mathrm{~mL})$ was added to the round bottom flask dropwise via an addition funnel within $1 \mathrm{~h}$ at $0{ }^{\circ} \mathrm{C}$. The resulting reaction mixture was then warmed up to room temperature and stirred for additional $2 \mathrm{~h}$ after removing the cooling bath. Ice-cooled water $(50 \mathrm{~mL})$ was poured into the reaction, followed by addition of $10 \% \mathrm{HCl}$ to adjust the solution to $\mathrm{pH}=2$. Yellow precipitate was formed during the acidification. After sedimentation, the solid was collected, washed with water $(50 \mathrm{~mL})$, and recrystallized with EtOH yielded a light-yellow flask solid 3 (1.42 g, 63\%).

m.p. $92-94{ }^{\circ} \mathrm{C} ; R_{f}=0.36\left(\right.$ EtOAc/hexane, 1:8). ${ }^{1} \mathrm{H}-\mathrm{NMR}\left(\mathrm{CDCl}_{3}, 400 \mathrm{MHz}\right) \delta 12.79(\mathrm{~s}$, $1 \mathrm{H}), 8.00-7.90(\mathrm{~m}, 2 \mathrm{H}), 7.70-7.60(\mathrm{~m}, 3 \mathrm{H}), 7.48(\mathrm{t}, J=8.6 \mathrm{~Hz}, 1 \mathrm{H}), 7.45-7.35(\mathrm{~m}, 3 \mathrm{H})$, $7.02(\mathrm{dd}, J=8.4,0.9 \mathrm{~Hz}, 1 \mathrm{H}), 6.93(\mathrm{t}, J=8.4 \mathrm{~Hz}, 1 \mathrm{H}) .{ }^{13} \mathrm{C}-\mathrm{NMR}\left(\mathrm{CDCl}_{3}, 100 \mathrm{MHz}\right) \delta$ 193.7, 163.5, 145.4, 136.3, 134.5, 130.9, 129.6, 129.0, 128.6, 120.0, 120.0, 118.8, 118.6. IR (ZnSe) 1639, 1618 $\mathrm{cm}^{-1}$. ESI-HRMS calcd for $\mathrm{C}_{15} \mathrm{H}_{13} \mathrm{O}_{2}\left(\mathrm{M}^{+}+1\right) 225.0910$, found 
<smiles>O=C1CC(c2ccccc2)Oc2ccccc21</smiles>

\section{2-phenylchroman-4-one (4)}

To a solution of $\mathbf{3}(87 \mathrm{mg}, 0.39 \mathrm{mmol})$ in EtOH ( $2 \mathrm{~mL})$ was added NaOAc (70 mg, 0.85 mmol, 2.2 equiv.), and the reaction mixture was stirred and refluxed for $24 \mathrm{~h}$. After cooling to room temperature, water $(10 \mathrm{~mL})$ was added to the mixture, and further extracted with EtOAc $(10 \mathrm{~mL} \times 3)$. The combined organic layers were washed with brine $(30 \mathrm{~mL})$, dried over anhydrous $\mathrm{MgSO}_{4}$, and concentrated. The crude product was purified by flash column chromatography $($ EtOAc/hexane $=1: 8)$ to give a pale yellow solid 4 (62 mg, $0.28 \mathrm{mmol}, 71 \%$ ).

m.p. 81-82 ${ }^{\circ} \mathrm{C} ; R_{f}=0.29\left(\right.$ EtOAc/hexane, 1:8). ${ }^{1} \mathrm{H}-\mathrm{NMR}\left(\mathrm{CDCl}_{3}, 400 \mathrm{MHz}\right) \delta 7.93(\mathrm{dd}$, $J=8.1,1.7 \mathrm{~Hz} 1 \mathrm{H}), 7.53-7.35(\mathrm{~m}, 6 \mathrm{H}), 7.07-7.02(\mathrm{~m}, 2 \mathrm{H}), 5.47$ (dd, $J=13.3,2.9 \mathrm{~Hz}$, $1 \mathrm{H}), 3.07(\mathrm{dd}, J=16.9,13.3 \mathrm{~Hz}, 1 \mathrm{H}), 2.88(\mathrm{dd}, J=16.9,2.9 \mathrm{~Hz}, 1 \mathrm{H}) .{ }^{13} \mathrm{C}-\mathrm{NMR}\left(\mathrm{CDCl}_{3}\right.$, $100 \mathrm{MHz}) \delta 191.9$, 161.5, 138.7, 136.1, 128.8, 128.7, 127.0, 126.1, 121.6, 120.9, 118.1, 79.5, 44.6. IR (ZnSe) 1683, $1606 \mathrm{~cm}^{-1}$. ESI-HRMS calcd for $\mathrm{C}_{15} \mathrm{H}_{13} \mathrm{O}_{2}\left(\mathrm{M}^{+}+1\right)$ 225.0910 , found 225.0886 .<smiles>O=C1c2ccccc2OC(c2ccccc2)C1Br</smiles>

\section{3-bromo-2-phenylchroman-4-one (5)}

To a solution of 4 (7.86 g, $35 \mathrm{mmol}$.) in $\mathrm{CCl}_{4}(90 \mathrm{~mL})$ was added $\mathrm{Br}_{2}(1.8 \mathrm{~mL}, 35 \mathrm{mmol}$, 1.0 equiv.) within $10 \mathrm{~min}$, and the reaction mixture was stirred for $2 \mathrm{~h}$ at room temperature. The solution was added sat. $\mathrm{Na}_{2} \mathrm{~S}_{2} \mathrm{O}_{3}(\mathrm{aq})(100 \mathrm{~mL})$, and extracted with $\operatorname{DCM}(50 \mathrm{~mL} \times 3)$. The combined organic layers were washed with sat. $\mathrm{NaCl}_{(\mathrm{aq})}(200$ $\mathrm{mL}$ ), dried over anhydrous $\mathrm{MgSO}_{4}$, and concentrated. The crude product was purified by flash column chromatography $(\mathrm{DCM} /$ hexane $=2: 3)$ to give a colorless liquid $\mathbf{5}(9.79$ g, $32 \mathrm{mmol}, 92 \%, 70: 30 \mathrm{dr})$.

$R_{f}=0.37(\mathrm{DCM} / \mathrm{hexane}, 2 / 3) .{ }^{1} \mathrm{H}-\mathrm{NMR}\left(\mathrm{CDCl}_{3}, 400 \mathrm{MHz}\right)$

cis-5 $\delta 7.99(\mathrm{dd}, J=8.0,1.6 \mathrm{~Hz}, 1 \mathrm{H}), 7.58(\mathrm{td}, J=8.5,1.7 \mathrm{~Hz}, 1 \mathrm{H}), 7.55-7.37$ (m, 5H), 
7.18-7.09 (m, 2H), 5.42 (br, 1H), 4.53 (d, $J=1.6 \mathrm{~Hz}, 1 \mathrm{H})$.

trans-5

$\delta 7.93(\mathrm{dd}, J=7.9,1.5 \mathrm{~Hz}, 1 \mathrm{H}), 7.56-7.50(\mathrm{~m}, 1 \mathrm{H}), 7.48-7.36(\mathrm{~m}, 5 \mathrm{H}), 7.10-7.04(\mathrm{~m}$, $2 \mathrm{H}), 5.56(\mathrm{~d}, J=8.6 \mathrm{~Hz}, 1 \mathrm{H}), 4.99(\mathrm{~d}, J=8.6 \mathrm{~Hz}, 1 \mathrm{H})$.

${ }^{13} \mathrm{C}-\mathrm{NMR}\left(\mathrm{CDCl}_{3}, 100 \mathrm{MHz}\right)$ (mixture) $\delta 186.3,185.2,160.4,160.0,136.9,2507$, 136.1, 135.6, 129.3, 128.9, 128.8, 128.5, 128.4, 128.0, 127.2, 126.0, 122.6, 122.3, 119.0, 118.1, 117.9, 83.8, 79.5, 51.9, 51.5. IR (ZnSe) 1692, $1607 \mathrm{~cm}^{-1}$ ESI-HRMS calcd for $\mathrm{C}_{15} \mathrm{H}_{12} \mathrm{O}_{2} \mathrm{Br}\left(\mathrm{M}^{+}+1\right)$ 303.0015, found 302.9992 .<smiles>CC(F)(F)C1C(=O)c2ccccc2OC1c1ccccc1</smiles>

\section{S-(4-oxo-2-phenylchroman-3-yl) ethanethioate (6)}

To a solution of 5 (1.34 g, $4.42 \mathrm{mmol})$ in anhydrous MeCN (10 mL) were added KSAc ( $0.63 \mathrm{~g}, 5.52 \mathrm{mmol}, 1.2$ equiv.), and 18-crown-6 (35 mg, $0.13 \mathrm{mmol}, 0.03$ equiv.). The resulting reaction mixture was stirred for $30 \mathrm{~min}$, and then concentrated via a rotary evaporator. The residue was taken up in DCM $(50 \mathrm{~mL})$, washed with water $(50 \mathrm{~mL} \times 2)$ and brine (50 mL x 1), respectively, and dried over anhydrous $\mathrm{MgSO}_{4}$ and concentrated. The crude product was purified by flash column chromatography $($ EtOAc/hexane $=1 / 8)$ to afford a yellow liquid $6(0.77 \mathrm{~g}, 58 \%, 70: 30 \mathrm{dr})$.

$R_{f}=0.22($ EtOAc/hexane, $1 / 8) .{ }^{1} \mathrm{H}-\mathrm{NMR}\left(\mathrm{CDCl}_{3}, 400 \mathrm{MHz}\right) \delta 7.94(\mathrm{td}, J=7.6,1.8 \mathrm{~Hz}$, 1H), 7.54-7.50 (m, 1H), 7.47-7.41 (m, 2H), 7.40-7.31 (m, 3H), 7.10-7.00 (m, 2H), 5.49 $(\mathrm{d}, J=11.1 \mathrm{~Hz}, 1 \mathrm{H}), 4.78(\mathrm{~d}, J=11.1 \mathrm{~Hz}, 1 \mathrm{H}), 2.24(\mathrm{~s}, 3 \mathrm{H}) .{ }^{13} \mathrm{C}-\mathrm{NMR}\left(\mathrm{CDCl}_{3}, 100\right.$ MHz) $\delta 192.4,191.1,188.7,187.8,160.8,160.6,136.7,136.6,135.6,129.2,128.5$, $128.3,128.0,128.9,127.6,126.2,122.3,122.1,120.5,119.8,118.2,118.1,82.9,80.2$, 53.3, 51.1, 30.3. IR (ZnSe) 1692, $1606 \mathrm{~cm}^{-1}$ ESI-HRMS calcd for $\mathrm{C}_{17} \mathrm{H}_{15} \mathrm{O}_{3} \mathrm{~S}\left(\mathrm{M}^{+}+1\right)$ 299.0736, found 299.0746 .<smiles>O=C(O)c1c(-c2ccccc2)oc2ccccc2c1=O</smiles>

S-(4-oxo-2-phenyl-4H-chromen-3-yl) ethanethioate (7) 
To a solution of 6 (210 $\mathrm{mg}, 0.7 \mathrm{mmol})$ in anhydrous 1,4-dioxane (3 mL) was added 2,3Dichloro-5,6-dicyano-1,4-benzoquinone (DDQ) (227 mg, $1 \mathrm{mmol}, 1.4$ equiv.), and the reaction mixture was refluxed for $16 \mathrm{~h}$. After that, the reaction was filtered and concentrated, followed by flash column chromatography $($ EtOAc/hexane $=1 / 4)$ to afford a white solid 7 (120 mg, 58\%).

m.p. $141-142{ }^{\circ} \mathrm{C} ; R_{f}=0.32($ EtOAc/hexane, $1 / 4) .{ }^{1} \mathrm{H}-\mathrm{NMR}\left(\mathrm{CDCl}_{3}, 400 \mathrm{MHz}\right) \delta 8.26$ $(\mathrm{dd}, J=7.9,1.3 \mathrm{~Hz}, 1 \mathrm{H}), 7.70(\mathrm{td}, J=8.3,1.5 \mathrm{~Hz}, 1 \mathrm{H}), 7.65$ (d, $J=7.0 \mathrm{~Hz}, 2 \mathrm{H}), 7.55-$ $7.41(\mathrm{~m}, 5 \mathrm{H}), 2.35(\mathrm{~s}, 1 \mathrm{H}) .{ }^{13} \mathrm{C}-\mathrm{NMR}\left(\mathrm{CDCl}_{3}, 100 \mathrm{MHz}\right) \delta 192.0,175.0,168.9,155.9$, 134.2, 132.8, 131.1, 129.0, 128.2, 126.6, 125.8, 122.7, 118.0, 112.1, 30.1. IR (ZnSe) 1704, 1651, $1615 \mathrm{~cm}^{-1}$ ESI-HRMS calcd for $\mathrm{C}_{17} \mathrm{H}_{13} \mathrm{O}_{3} \mathrm{~S}\left(\mathrm{M}^{+}+1\right) 297.0580$, found 297.0580 . 


\section{General Procedure for the synthesis of SSF, FSSF, and NSSF}<smiles>O=c1cc(-c2ccc(F)cc2)oc2ccccc12</smiles>

2a, $\quad \mathrm{R}=\mathrm{H}$,

2b, $\mathrm{R}=\mathrm{CF}_{3}$,

2c, $\mathrm{R}=\mathrm{NEt}_{2}$,
(1) LDA, THF, $-78^{\circ} \mathrm{C}$,

(2) $S_{8}$,

(3) $10 \% \mathrm{HCl}$ or $5 \%$ citric acid

(4) $\mathrm{I}_{2}$, TEA, $\mathrm{MeOH} / \mathrm{CHCl}_{3}$

A solution of pre-dried diisopropylamine $(0.38 \mathrm{~mL}, 2.7 \mathrm{mmol}, 1.2$ equiv. $)$ in anhydrous and degassed THF $(10 \mathrm{~mL})$ was stirred and cooled to $-78^{\circ} \mathrm{C}$ (acetone/dry ice bath) for $10 \mathrm{~min}$, followed by the addition of ${ }^{n}$ butyl lithium (1.69 $\mathrm{M}$ in hexanes, $1.6 \mathrm{~mL}, 2.7$ mmol, 1.2 equiv.) within $5 \mathrm{~min}$ and stirred for additional $10 \mathrm{~min}$. at $-78{ }^{\circ} \mathrm{C}$. To this freshly prepared LDA solution was added compound $\mathbf{2 a}$ or $\mathbf{2 b}$, or $\mathbf{2 c}(2.3 \mathrm{mmol})$ dissolving in anhydrous THF $(5 \mathrm{~mL})$ within $5 \mathrm{~min}$, respectively and stirred for additional $30 \mathrm{~min}$ while kept at $-78{ }^{\circ} \mathrm{C}$, subsequent addition of sulphur powder $(88 \mathrm{mg}$, $2.75 \mathrm{mmol}, 1.2$ equiv.). After stirring for $1 \mathrm{hr}$. at $-78^{\circ} \mathrm{C}$, the mixture was warmed up to room temperature and poured into the degassed water $(30 \mathrm{~mL})$, followed by washing with the degassed diethyl ether $(20 \mathrm{~mL} \times 2)$. The organic layers were discarded and the aqueous layers were collected and acidified by either degassed $10 \% \mathrm{HCl}_{(\mathrm{aq})}$ or $5 \%$ citric $\operatorname{acid}_{(\mathrm{aq})}$. The precipitate formed and the degassed EtOAc $(10 \mathrm{~mL} \times 3)$ was added to dissolve the precipitate and extracted via a separatory funnel, followed by extracted with DCM (15 mL x 2) The combined organic layers were dried over anhydrous $\mathrm{MgSO}_{4}$, and concentrated to obtain the mixture of mercaptoflavone (3TF or 3FTF or 3NTF) and disulfide flavone (SSF or FSSF or NSSF), respectively, via a rotary evaporator. To the mixture in $50 \% \mathrm{MeOH} / \mathrm{CHCl}_{3}(5 \mathrm{~mL})$ was added saturated iodine in $\mathrm{MeOH}$ dropwise at room temperature. As the brown color of iodine persisted during the addition, sat. $\mathrm{Na}_{2} \mathrm{~S}_{2} \mathrm{O}_{3 \text { (aq) }}(10 \mathrm{~mL})$ was added and extracted with DCM (10 mL x 3), and the combined organic layers were dried over anhydrous $\mathrm{MgSO}_{4}$, and concentrated via a rotary evaporator. The residue was purified by flash column chromatography to afford disulfide flavones respectively. 
<smiles>O=c1c(SSc2c(-c3ccccc3)oc3ccccc3c2=O)c(-c2ccccc2)oc2ccccc12</smiles>

3,3'-disulfanediylbis(2-phenyl-4H-chromen-4-one) (SSF)

$49 \%$ yield. White solid. m.p. 271-272 ${ }^{\circ} \mathrm{C} ; R_{f}=0.31(\mathrm{MeOH} / \mathrm{DCM}, 1 / 50) .{ }^{1} \mathrm{H}-\mathrm{NMR}$ $\left(\mathrm{CDCl}_{3}, 400 \mathrm{MHz}\right) \delta 8.05(\mathrm{dd}, J=8.0,1.8 \mathrm{~Hz}, 1 \mathrm{H}), 7.67(\mathrm{td}, J=8.6,1.5 \mathrm{~Hz}, 1 \mathrm{H}), 7.52-$ $7.49(\mathrm{~m}, 2 \mathrm{H}), 7.42-7.34(\mathrm{~m}, 2 \mathrm{H}), 7.06-7.00(\mathrm{~m}, 3 \mathrm{H}) .{ }^{13} \mathrm{C}-\mathrm{NMR}\left(\mathrm{CDCl}_{3}, 100 \mathrm{MHz}\right) \delta$ 175.0, 167.6, 155.6, 133.8, 132.2, 130.6, 129.6, 127.4, 126.6, 125.3, 122.7, 118.1, 117.7. IR $(\mathrm{ZnSe}) 1732,1646,1613 \mathrm{~cm}^{-1}$. ESI-HRMS calcd for $\mathrm{C}_{30} \mathrm{H}_{19} \mathrm{O}_{4} \mathrm{~S}_{2}\left(\mathrm{M}^{+}+1\right) 507.0719$, found 507.0760 .<smiles>O=c1c(SSc2c(-c3ccc(C(F)(F)F)cc3)oc3ccccc3c2=O)c(-c2ccc(C(F)(F)F)cc2)oc2ccccc12</smiles>

3,3'-disulfanediylbis(2-(4-(trifluoromethyl)phenyl)-4H-chromen-4-one) (FSSF) $34 \%$ yield. White solid. m.p. 291-292 ${ }^{\circ} \mathrm{C} ; R_{f}=0.35(\mathrm{MeOH} / \mathrm{DCM}, 1 / 50) .{ }^{1} \mathrm{H}-\mathrm{NMR}$ $\left(\mathrm{CDCl}_{3}, 400 \mathrm{MHz}\right) \delta 8.02(\mathrm{~d}, J=8.3 \mathrm{~Hz}, 2 \mathrm{H}), 7.71(\mathrm{t}, J=7.6 \mathrm{~Hz}, 2 \mathrm{H}), 7.63(\mathrm{~d}, J=8.3$ $\mathrm{Hz}, 4 \mathrm{H}), 7.44-7.38(\mathrm{~m}, 4 \mathrm{H}), 7.32(\mathrm{~d}, J=8.3 \mathrm{~Hz}, 4 \mathrm{H}) .{ }^{13} \mathrm{C}-\mathrm{NMR}\left(\mathrm{CDCl}_{3}, 100 \mathrm{MHz}\right) \delta$ 174.5, 166.1, 155.4, 135.4, 134.3, 132.4 (q, $J=32.6 \mathrm{~Hz},{ }^{2} \mathrm{~J}_{\mathrm{CF}}$ ), 130.1, 126.5, 126.1, $124.6\left(\mathrm{q}, J=4.0 \mathrm{~Hz},{ }^{3} \mathrm{~J}_{\mathrm{CF}}\right), 122.6,118.7,117.6 .{ }^{19} \mathrm{~F}-\mathrm{NMR}\left(\mathrm{CDCl}_{3}, 376 \mathrm{MHz}\right) \delta-62.9$. IR (ZnSe) 1653, 1613, $1324 \mathrm{~cm}^{-1}$. ESI-HRMS calcd for $\mathrm{C}_{32} \mathrm{H}_{17} \mathrm{~F}_{6} \mathrm{O}_{4} \mathrm{~S}_{2}\left(\mathrm{M}^{+}+1\right)$ 643.0467, found 643.0511. 
<smiles>CCN(CC)c1ccc(-c2oc3ccccc3c(=O)c2SSc2c(-c3ccc(N(CC)CC)cc3)oc3ccccc3c2=O)cc1</smiles>

\section{3,3'-disulfanediylbis(2-(4-(diethylamino)phenyl)-4H-chromen-4-one) (NSSF)}

$41 \%$ yield. Yellow solid. m.p. $269-270{ }^{\circ} \mathrm{C} ; R_{f}=0.20(\mathrm{MeOH} / \mathrm{DCM}, 1 / 50) .{ }^{1} \mathrm{H}-\mathrm{NMR}$ $\left(\mathrm{CDCl}_{3}, 400 \mathrm{MHz}\right) \delta 8.02(\mathrm{~d}, J=7.0 \mathrm{~Hz}, 2 \mathrm{H}), 7.58-7.52(\mathrm{~m}, 6 \mathrm{H}), 7.29(\mathrm{~d}, J=8.6 \mathrm{~Hz}$, 2H), $7.21(\mathrm{~d}, J=7.0 \mathrm{~Hz}, 2 \mathrm{H}), 6.19(\mathrm{br}, 4 \mathrm{H}), 3.13$ (q, $J=7.0 \mathrm{~Hz}, 8 \mathrm{H}), 1.06$ (t, $J=6.9$ $\mathrm{Hz}, 12 \mathrm{H}) .{ }^{13} \mathrm{C}-\mathrm{NMR}\left(\mathrm{CDCl}_{3}, 100 \mathrm{MHz}\right) \delta 175.4,155.4,132.8,131.7,126.4,124.5$, 122.6, 117.3, 109.4, 44.3, 12.6. IR (ZnSe) 1734, $1602 \mathrm{~cm}^{-1}$. ESI-HRMS calcd for $\mathrm{C}_{38} \mathrm{H}_{37} \mathrm{~N}_{2} \mathrm{O}_{4} \mathrm{~S}_{2}\left(\mathrm{M}^{+}+1\right) 649.2189$, found 649.2238.

\section{General Procedure for the synthesis of 3TF, 3FTF, and 3NTF}

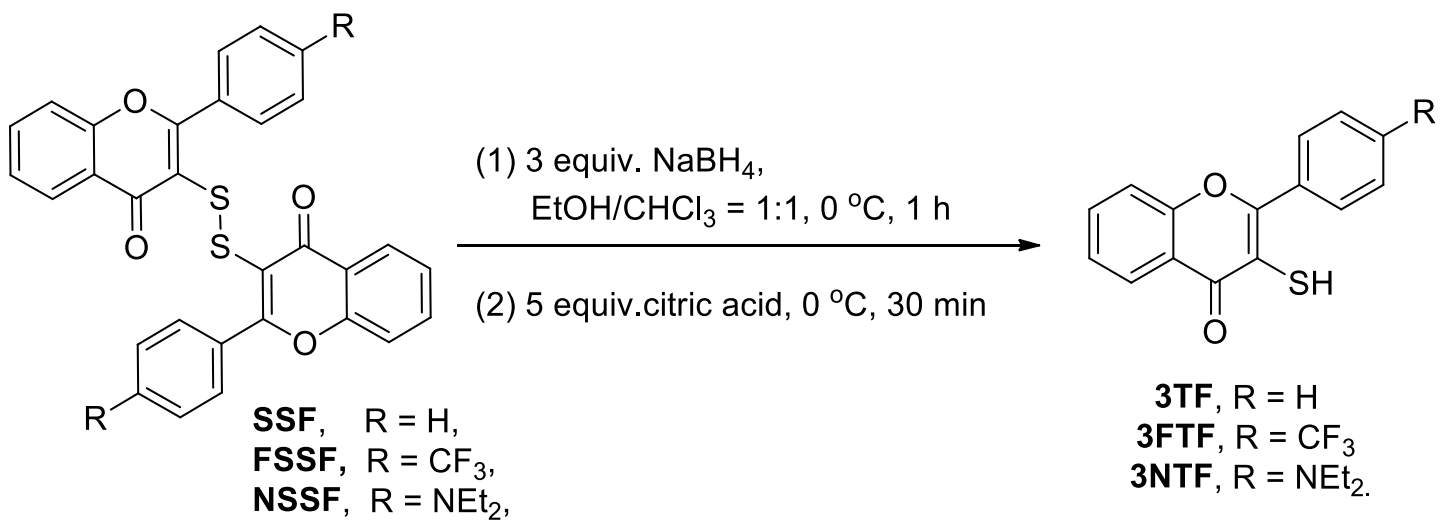

A stirred solution or suspension of disulfide $(0.1 \mathrm{mmol})$ in $50 \% \mathrm{EtOH} / \mathrm{CHCl}_{3}(2 \mathrm{~mL})$ was kept in $0{ }^{\circ} \mathrm{C}$ and added sodium borohydride ( 3 equiv.) for $1 \mathrm{~h}$. Until the turbid solution became clear was added citric acid (5 equiv.) and stirred for additional $30 \mathrm{~min}$ at $0{ }^{\circ} \mathrm{C}$. The solution was added degassed water $(2 \mathrm{~mL})$ and extracted with degassed EtOAc $(1 \mathrm{~mL} \times 2)$. The combined organic layers were sequentially washed with degassed $\mathrm{NH}_{4} \mathrm{Cl}_{(\mathrm{aq})}(2 \mathrm{~mL})$ and degassed sat. $\mathrm{NaCl}_{(\mathrm{aq})}(2 \mathrm{~mL})$, dried over anhydrous $\mathrm{MgSO}_{4}$, and concentrated. Recrystallization of the residue using the freeze-pumpthawing degassed EtOAc at a $-4{ }^{\circ} \mathrm{C}$ refrigerator afforded single-crystal quality of mercaptoflavone. 
<smiles>O=c1c(S)c(-c2ccccc2)oc2ccccc12</smiles>

3TF

3-mercapto-2-phenyl-4H-chromen-4-one (3TF)

Quantitative yield. Yellow solid. m.p. $260^{\circ} \mathrm{C}$ (decomposition); $R_{f}=0.25$ (DCM/hexane, 3/2). ${ }^{1} \mathrm{H}-\mathrm{NMR}\left(\mathrm{CDCl}_{3}, 400 \mathrm{MHz}\right) \delta 8.27(\mathrm{~d}, J=7.5 \mathrm{~Hz}, 1 \mathrm{H}), 7.90(\mathrm{~d}, J=7.7 \mathrm{~Hz}, 2 \mathrm{H})$, $7.68(\mathrm{t}, J=6.9 \mathrm{~Hz}, 1 \mathrm{H}), 7.57-7.48(\mathrm{~m}, 4 \mathrm{H}), 7.43(\mathrm{t}, J=7.7 \mathrm{~Hz}, 1 \mathrm{H}), 5.24(\mathrm{~s}, 1 \mathrm{H}) .{ }^{13} \mathrm{C}-$ $\mathrm{NMR}\left(\mathrm{CDCl}_{3}, 100 \mathrm{MHz}\right) \delta 173.4,156.8,155.5,133.8,133.4,130.8,128.6,128.5,126.5$, 125.2, 120.0, 118.0, 30.9. IR (ZnSe) 2500, 1628, $1612 \mathrm{~cm}^{-1}$. ESI-HRMS calcd for $\mathrm{C}_{15} \mathrm{H}_{11} \mathrm{O}_{2} \mathrm{~S}\left(\mathrm{M}^{+}+1\right) 255.0474$, found 255.0494.<smiles>O=c1c(S)c(-c2ccc(C(F)(F)F)cc2)oc2ccccc12</smiles>

3FTF

\section{3-mercapto-2-(4-(trifluoromethyl)phenyl)-4H-chromen-4-one (3FTF)}

Quantitative yield. Pale Yellow solid. m.p. $130-131^{\circ} \mathrm{C} ; R_{f}=0.39$ (DCM/hexane, 1/1). ${ }^{1} \mathrm{H}-\mathrm{NMR}\left(\mathrm{CDCl}_{3}, 400 \mathrm{MHz}\right) \delta 8.28(\mathrm{dd}, J=8.3,1.6 \mathrm{~Hz}, 1 \mathrm{H}), 8.04(\mathrm{~d}, J=8.3 \mathrm{~Hz}, 2 \mathrm{H})$, $7.81(\mathrm{~d}, J=8.3 \mathrm{~Hz}, 2 \mathrm{H}), 7.71(\mathrm{td}, J=7.5,1.7 \mathrm{~Hz}, 1 \mathrm{H}), 7.51(\mathrm{~d}, J=8.2 \mathrm{~Hz}, 1 \mathrm{H}), 7.46$ $(\mathrm{td}, J=7.5,1.1 \mathrm{~Hz}, 1 \mathrm{H}), 5.33(\mathrm{~s}, 1 \mathrm{H}) .{ }^{13} \mathrm{C}-\mathrm{NMR}\left(\mathrm{CDCl}_{3}, 100 \mathrm{MHz}\right) \delta 173.2,155.5$, $154.8,136.8,134.1,132.6,130.1,129.0,126.6,126.5,125.7\left(\mathrm{q}, J=4.0 \mathrm{~Hz}, 1 \mathrm{H},{ }^{3} \mathrm{~J}_{\mathrm{CF}}\right)$, 125.5, 124.6, 119.8, 118.3, 118.0, 117.6. ${ }^{19} \mathrm{~F}-\mathrm{NMR}\left(\mathrm{CDCl}_{3}, 376 \mathrm{MHz}\right) \delta$-63.0. IR $(\mathrm{ZnSe})$ 2534, 1632, $1614 \mathrm{~cm}^{-1}$. ESI-HRMS calcd for $\mathrm{C}_{16} \mathrm{H}_{10} \mathrm{~F}_{3} \mathrm{O}_{2} \mathrm{~S}\left(\mathrm{M}^{+}+1\right) 323.0354$, found 323.0323 .<smiles>CN(C)c1ccc(-c2oc3ccccc3c(=O)c2S)cc1</smiles>

2-(4-(diethylamino)phenyl)-3-mercapto-4H-chromen-4-one (3NTF)

Quantitative yield. Dark red brown solid. m.p. $120-121{ }^{\circ} \mathrm{C} ; R_{f}=0.47$ (DCM/hexane, 3/2). ${ }^{1} \mathrm{H}-\mathrm{NMR}\left(\mathrm{CDCl}_{3}, 400 \mathrm{MHz}\right) \delta 8.24(\mathrm{dd}, J=8.0,1.5 \mathrm{~Hz}, 1 \mathrm{H}), 7.88(\mathrm{~d}, J=8.2 \mathrm{~Hz}$, 
2H), 7.64 (t, $J=7.6 \mathrm{~Hz}, 1 \mathrm{H}), 7.49$ (d, $J=8.2 \mathrm{~Hz}, 1 \mathrm{H}), 7.35$ (t, $J=8.2 \mathrm{~Hz}, 1 \mathrm{H}), 6.78$ (br, $2 \mathrm{H}), 5.38(\mathrm{~s}, 1 \mathrm{H}), 3.44(\mathrm{q}, J=6.9 \mathrm{~Hz}, 4 \mathrm{H}), 1.22(\mathrm{t}, J=7.0 \mathrm{~Hz}, 6 \mathrm{H}) \cdot{ }^{13} \mathrm{C}-\mathrm{NMR}\left(\mathrm{CDCl}_{3}\right.$, $100 \mathrm{MHz}) \delta 173.2,155.4,155.2,133.2,131.7,130.2,126.3,124.8,120.0,117.7,117.4$, 110.8, 44.8, 12.4. IR (ZnSe) 2505, 1710, $1601 \mathrm{~cm}^{-1}$. ESI-HRMS calcd for $\mathrm{C}_{19} \mathrm{H}_{20} \mathrm{NO}_{2} \mathrm{~S}$ $\left(\mathrm{M}^{+}+1\right)$ 326.1209, found 326.1179.<smiles>CSc1c(-c2ccc(N(C)C)cc2)oc2ccccc2c1=O</smiles>

3MeNTF

\section{3-(methylthio)-2-phenyl-4H-chromen-4-one (3MeNTF)}

From 3NTF: To a solution of 3NTF (10 mg, $0.04 \mathrm{mmol})$ in anhydrous THF $(0.5 \mathrm{~mL})$ were added methyl iodide ( $7 \mu \mathrm{L}, 0.11 \mathrm{mmol}, 2.8$ equiv.), and triethylamine ( $15 \mu \mathrm{L}, 0.11$ mmol, 2.8 equiv.), and the reaction mixture was stirred at room temperature for $1 \mathrm{~h}$. After that, water $(1 \mathrm{~mL})$ was added to the reaction followed by the extraction with EtOAc $(1 \mathrm{~mL} \times 3)$. The combined organic layers were washed with sat. $\mathrm{NaCl}_{(\mathrm{aq})}(3 \mathrm{~mL})$ and dried over anhydrous $\mathrm{MgSO}_{4}$ and concentrated. The residue was then purified by flash column chromatography (100\% DCM) to afford a yellow solid 3MeNTF (12 mg, 91\%).

From NSSF: To a stirred suspension of NSSF $(20.0 \mathrm{mg}, 0.03 \mathrm{mmol})$ in $50 \%$ $\mathrm{EtOH} / \mathrm{CHCl}_{3}(1 \mathrm{~mL})$ was kept in $0{ }^{\circ} \mathrm{C}$ and added sodium borohydride $(3.4 \mathrm{mg}, 0.09$ mmol, 3 equiv.) for $1 \mathrm{~h}$. Until the turbid solution became clear was added $\mathrm{MeI}(9 \mu \mathrm{L}$, $0.15 \mathrm{mmol}, 5$ equiv.) and stirred for additional $30 \mathrm{~min}$ at $0{ }^{\circ} \mathrm{C}$. The solution was added water $(1 \mathrm{~mL})$ and extracted with $\mathrm{CHCl}_{3}(1 \mathrm{~mL} \times 3)$. The combined organic layers were washed with degassed sat. $\mathrm{NaCl}_{(\mathrm{aq})}(2 \mathrm{~mL})$, dried over anhydrous $\mathrm{MgSO}_{4}$, and concentrated. The residue was then purified by flash column chromatography $(100 \%$ DCM) to afford a yellow solid 3MeNTF (18 mg, 86\%).

m.p. $94-95{ }^{\circ} \mathrm{C} ; R_{f}=0.30$ (DCM). ${ }^{1} \mathrm{H}-\mathrm{NMR}\left(\mathrm{CDCl}_{3}, 400 \mathrm{MHz}\right) \delta 8.23(\mathrm{dd}, J=7.9,1.6$ $\mathrm{Hz}, 1 \mathrm{H}), 7.79$ (d, $J=9.0 \mathrm{~Hz}, 2 \mathrm{H}), 7.62(\mathrm{td}, J=7.7,1.6 \mathrm{~Hz}, 1 \mathrm{H}), 7.43$ (d, $J=8.2 \mathrm{~Hz}$, 1H), 7.37 (td, $J=7.7,1.6 \mathrm{~Hz}, 1 \mathrm{H}), 6.71(\mathrm{~d}, 7.4 \mathrm{~Hz}, 2 \mathrm{H}), 3.43$ (q, $J=7.3 \mathrm{~Hz}, 4 \mathrm{H}), 2.34$ $(\mathrm{s}, 3 \mathrm{H}), 1.22(\mathrm{t}, J=6.8 \mathrm{~Hz}, 6 \mathrm{H}) .{ }^{13} \mathrm{C}-\mathrm{NMR}\left(\mathrm{CDCl}_{3}, 100 \mathrm{MHz}\right) \delta 176.5,166.2,155.6$, 133.3, 131.6, 126.1 124.9, 122.5, 117.6, 115.4, 110.0, 53.4, 44.4, 17.6, 12.5. IR (ZnSe) 1720, 1637, $1603 \mathrm{~cm}^{-1}$. ESI-HRMS calcd for $\mathrm{C}_{20} \mathrm{H}_{22} \mathrm{NO}_{2} \mathrm{~S}\left(\mathrm{M}^{+}+1\right) 340.1371$, found 340.1389 . 


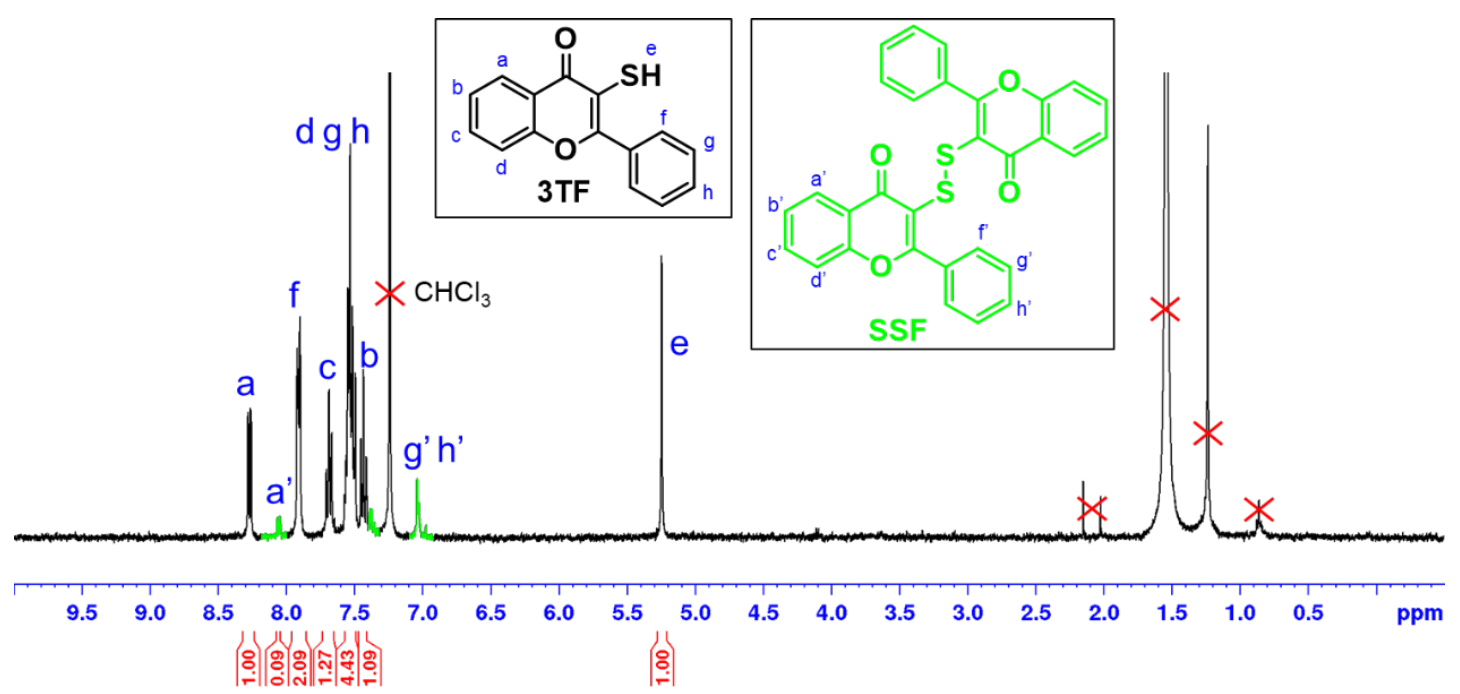

Figure S1. ${ }^{1} \mathrm{H}-\mathrm{NMR}$ spectrum in $\mathrm{CDCl}_{3}$ acquired from route 1 in scheme 1 indicating the presence of $\mathbf{3 T F}$ and a small amount of SSF. The peaks highlighted in green colour corresponded to SSF.

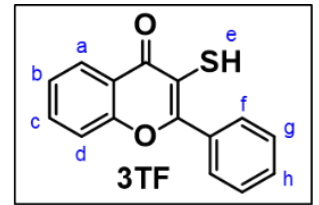

(a)

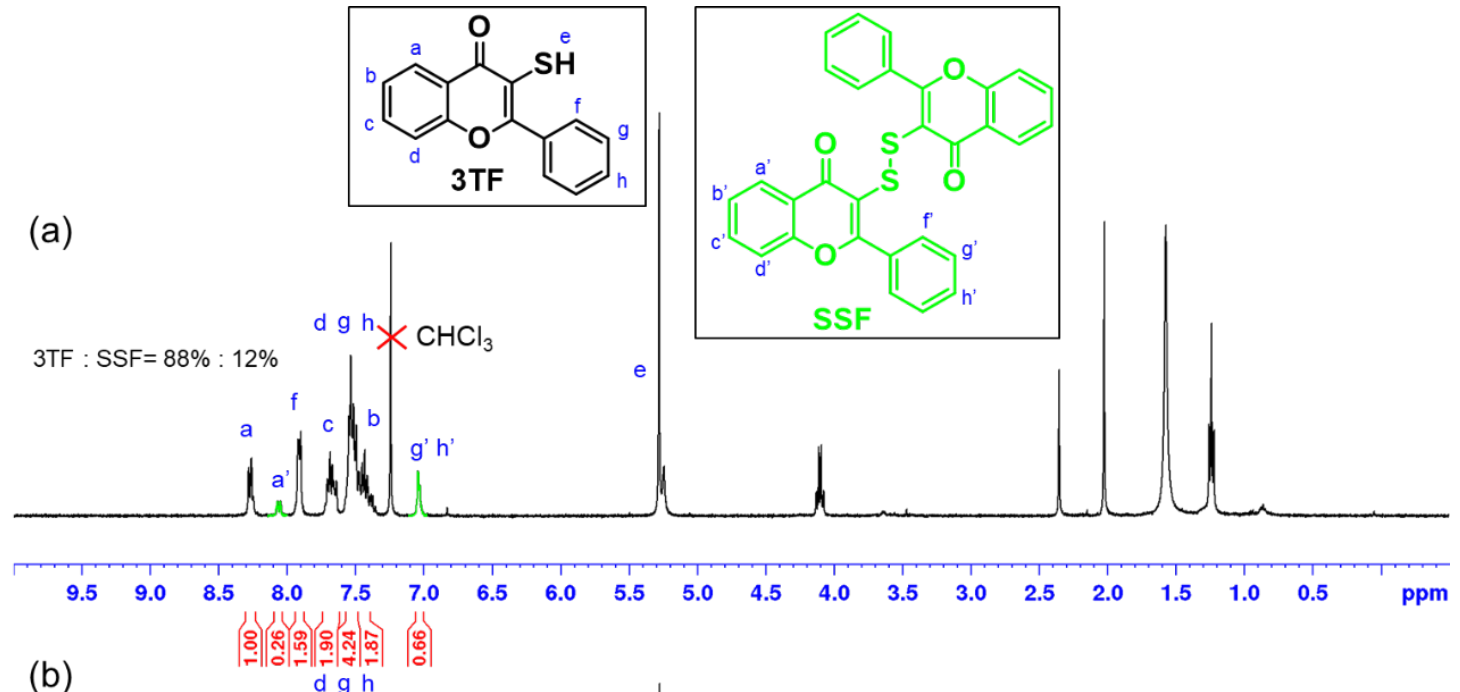

(b)
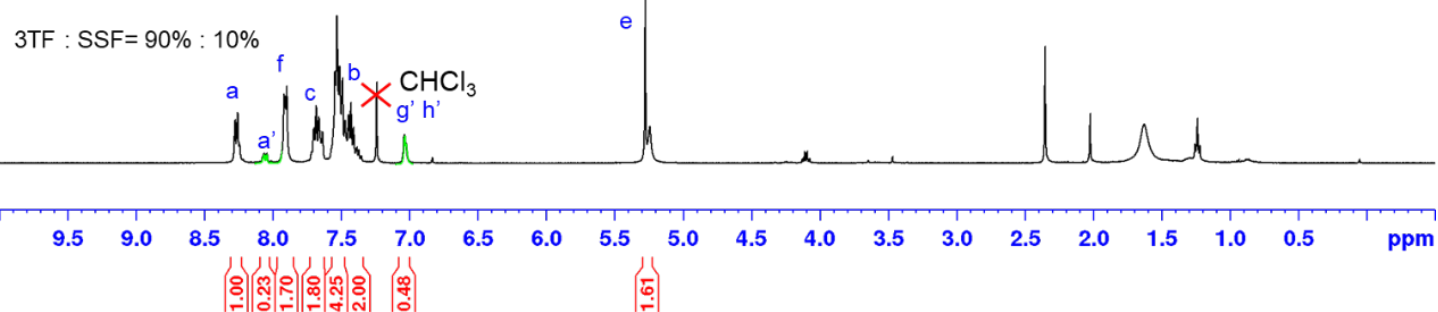

Figure S2. The crude spectra (a) and (b) in $\mathrm{CDCl}_{3}$ acquired from step $\mathrm{h}$ of the route 2 in scheme 1 after acidified with $\mathrm{HCl}$ or citric acid, respectively, indicated the presence of 3 TF as the major product accompanied with SSF as the minor product. The peaks highlighted in green colour corresponded to SSF. 

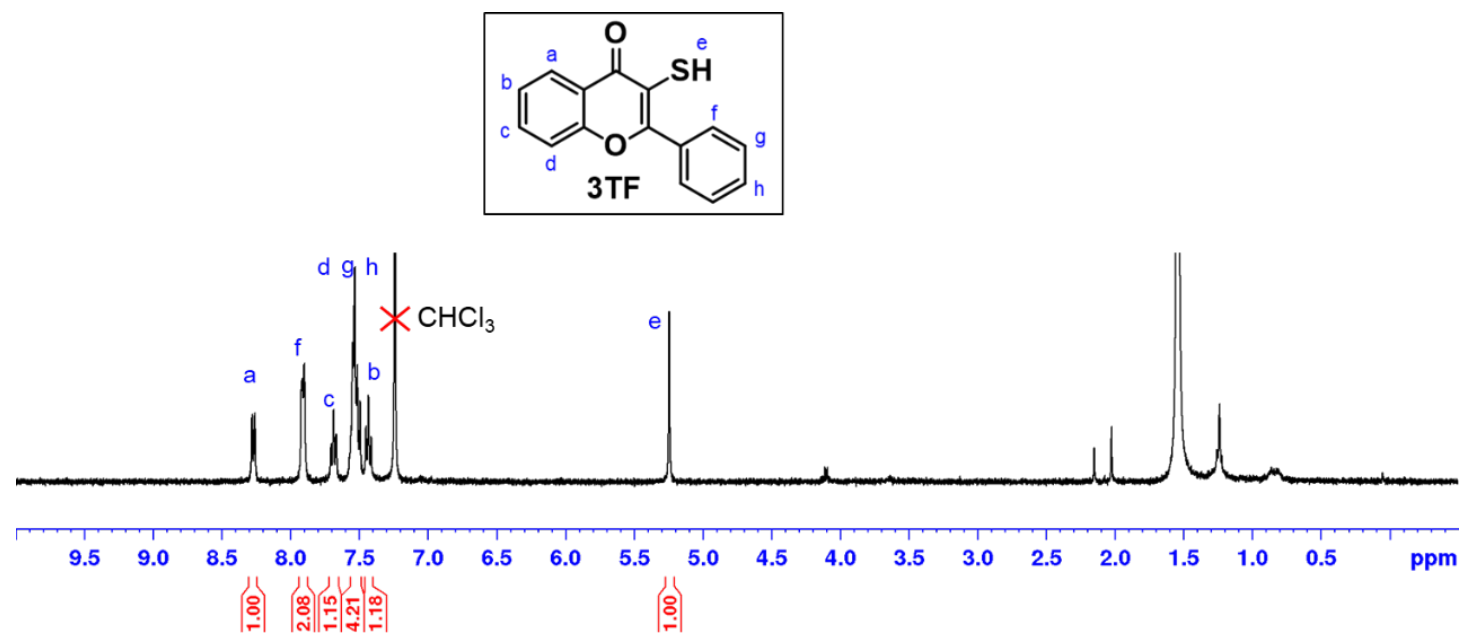

Figure S3. The crude spectrum in $\mathrm{CDCl}_{3}$ acquired from step $\mathrm{b}$ of the route 1 in scheme $\mathbf{1}$ indicated only $\mathbf{3 T F}$ present and no SSF evidenced after acidified with citric acid. 

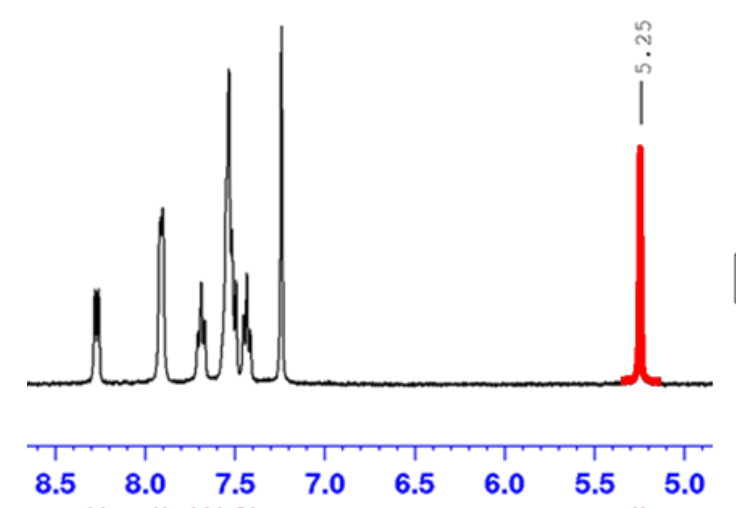

|ำ:
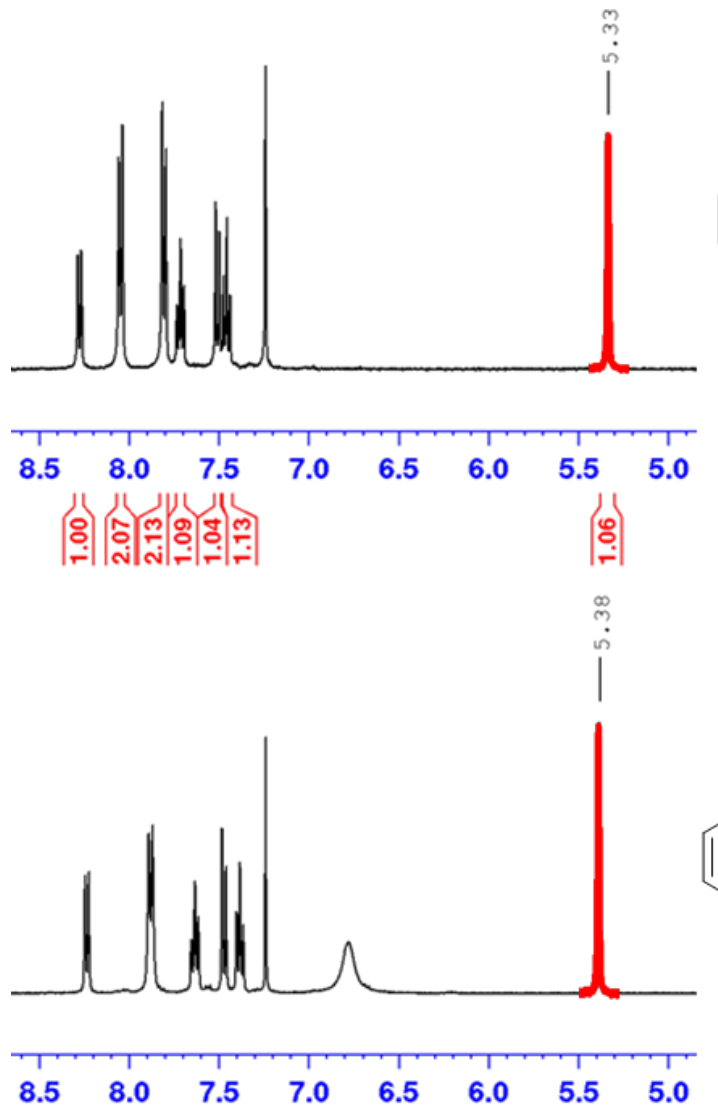

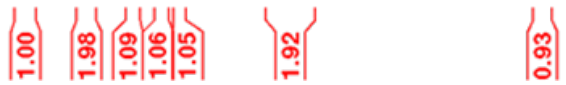<smiles>O=c1c(S)c(-c2ccccc2)oc2ccccc12</smiles>

3TF<smiles></smiles>

3FTF

Figure S4. The sharp singlet peaks corresponding to the thiol protons of 3TF, 3FTF, and $\mathbf{3 N T F}$ were evidenced in ${ }^{1} \mathrm{H}-\mathrm{NMR}$. 


\section{Structure Characterization}

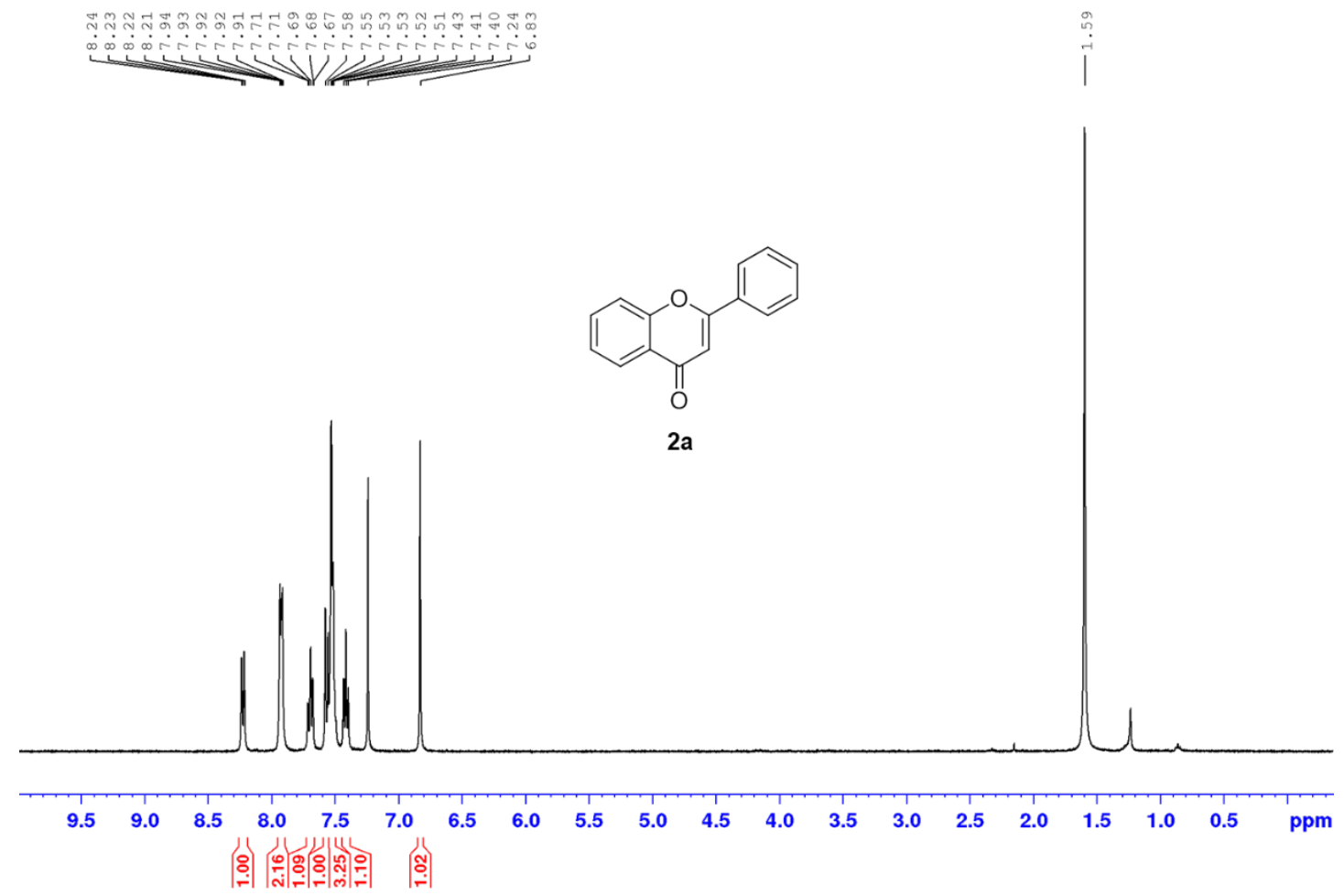

Figure S5. ${ }^{1} \mathrm{H} \mathrm{NMR}$ of $\mathbf{2 a}$ in $\mathrm{CDCl}_{3}$.
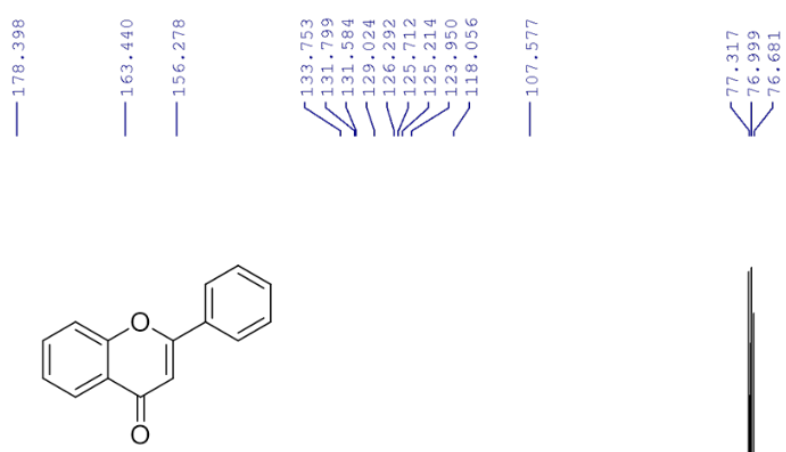

2a

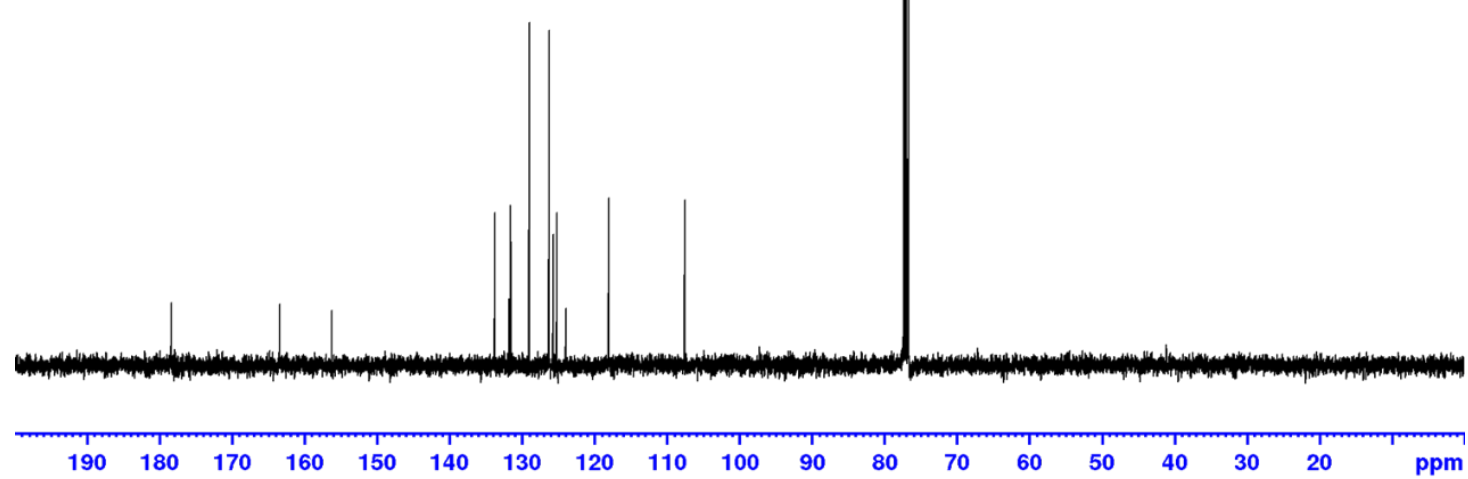

Figure S6. ${ }^{13} \mathrm{C}$ NMR of $\mathbf{2 a}$ in $\mathrm{CDCl}_{3}$. 


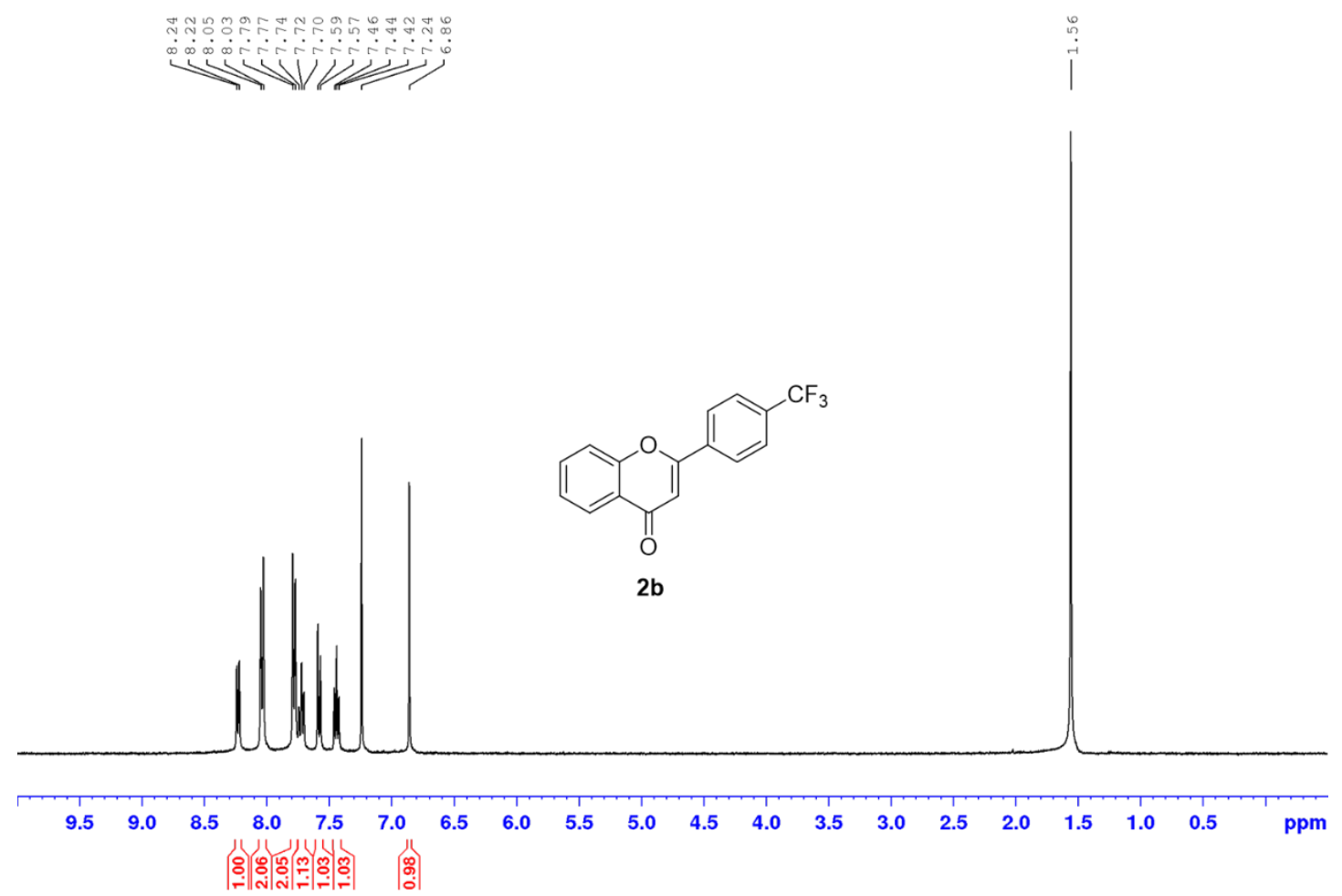

Figure S7. ${ }^{1} \mathrm{H}$ NMR of $\mathbf{2 b}$ in $\mathrm{CDCl}_{3}$.

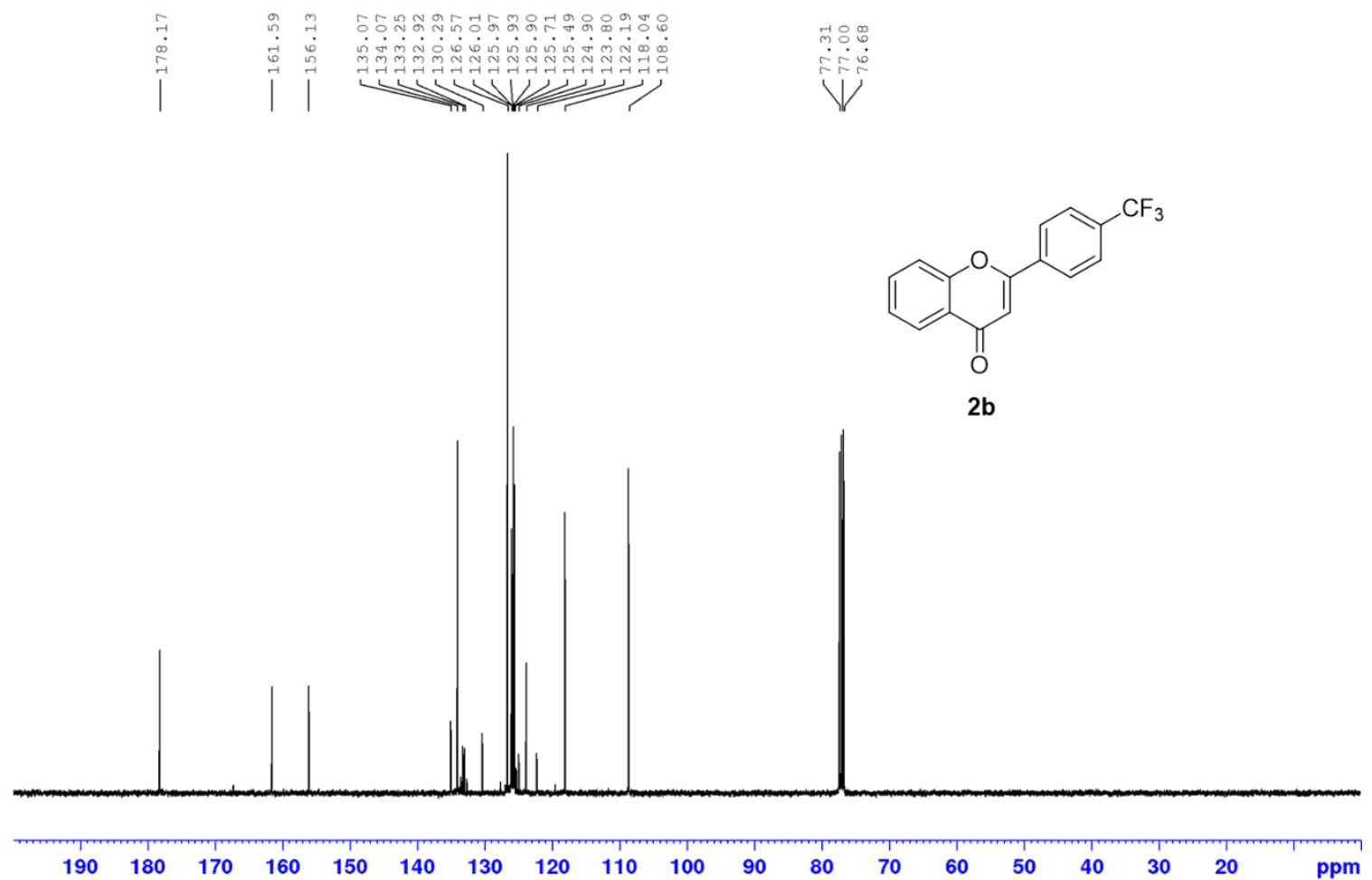

Figure S8. ${ }^{13} \mathrm{C}$ NMR of $\mathbf{2 b}$ in $\mathrm{CDCl}_{3}$. 


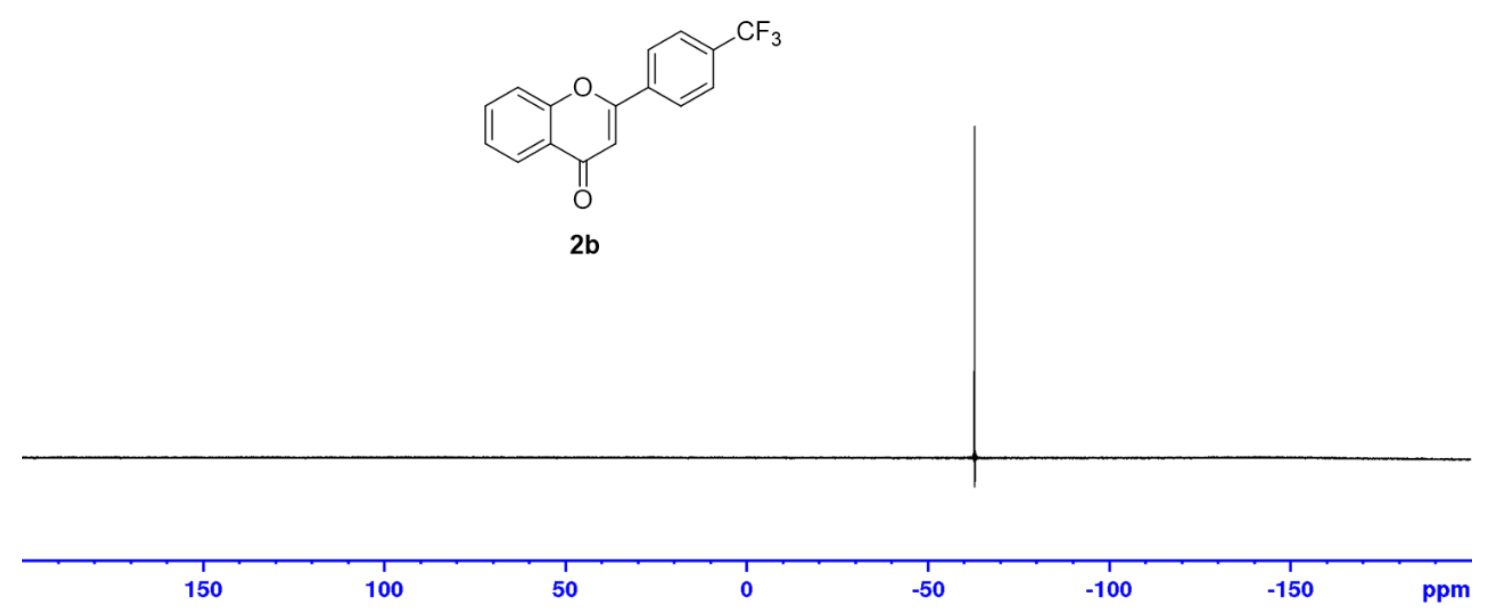

Figure S9. ${ }^{19} \mathrm{~F}$ NMR of $\mathbf{2 b}$ in $\mathrm{CDCl}_{3}$. 


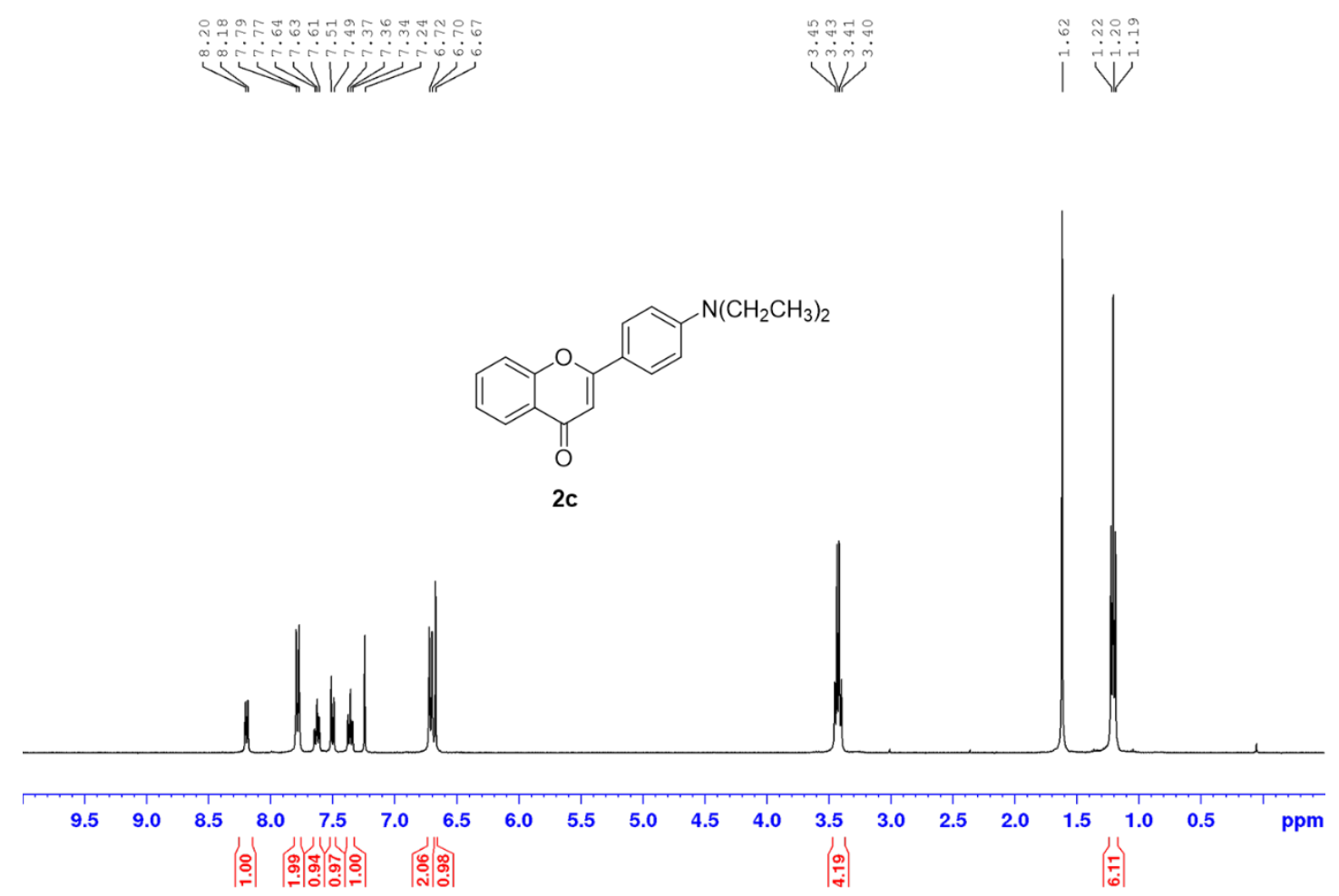

Figure S10. ${ }^{1} \mathrm{H} \mathrm{NMR}$ of $2 \mathrm{c}$ in $\mathrm{CDCl}_{3}$.

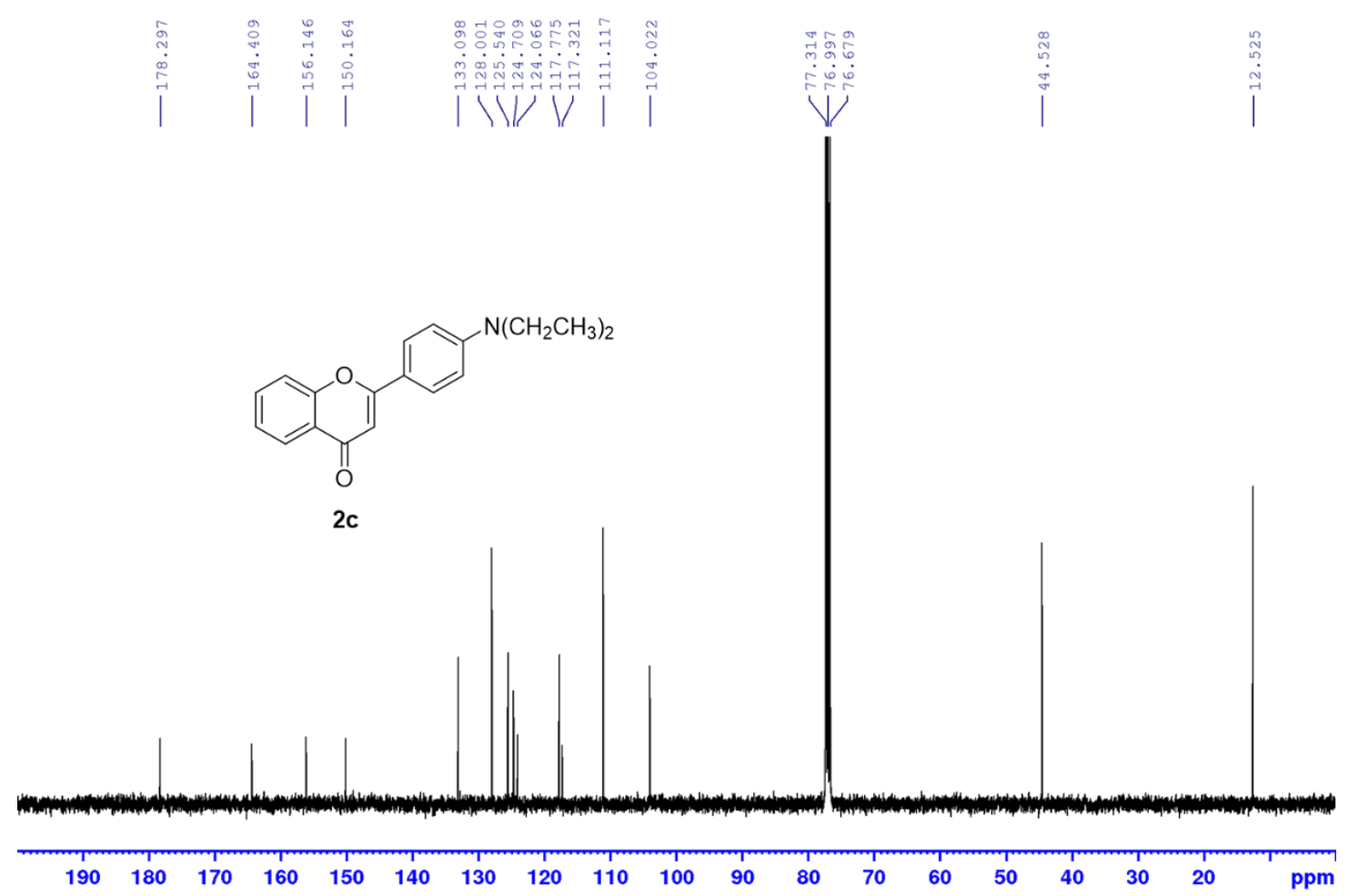

Figure S11. ${ }^{13} \mathrm{C}$ NMR of $2 \mathrm{c}$ in $\mathrm{CDCl}_{3}$. 


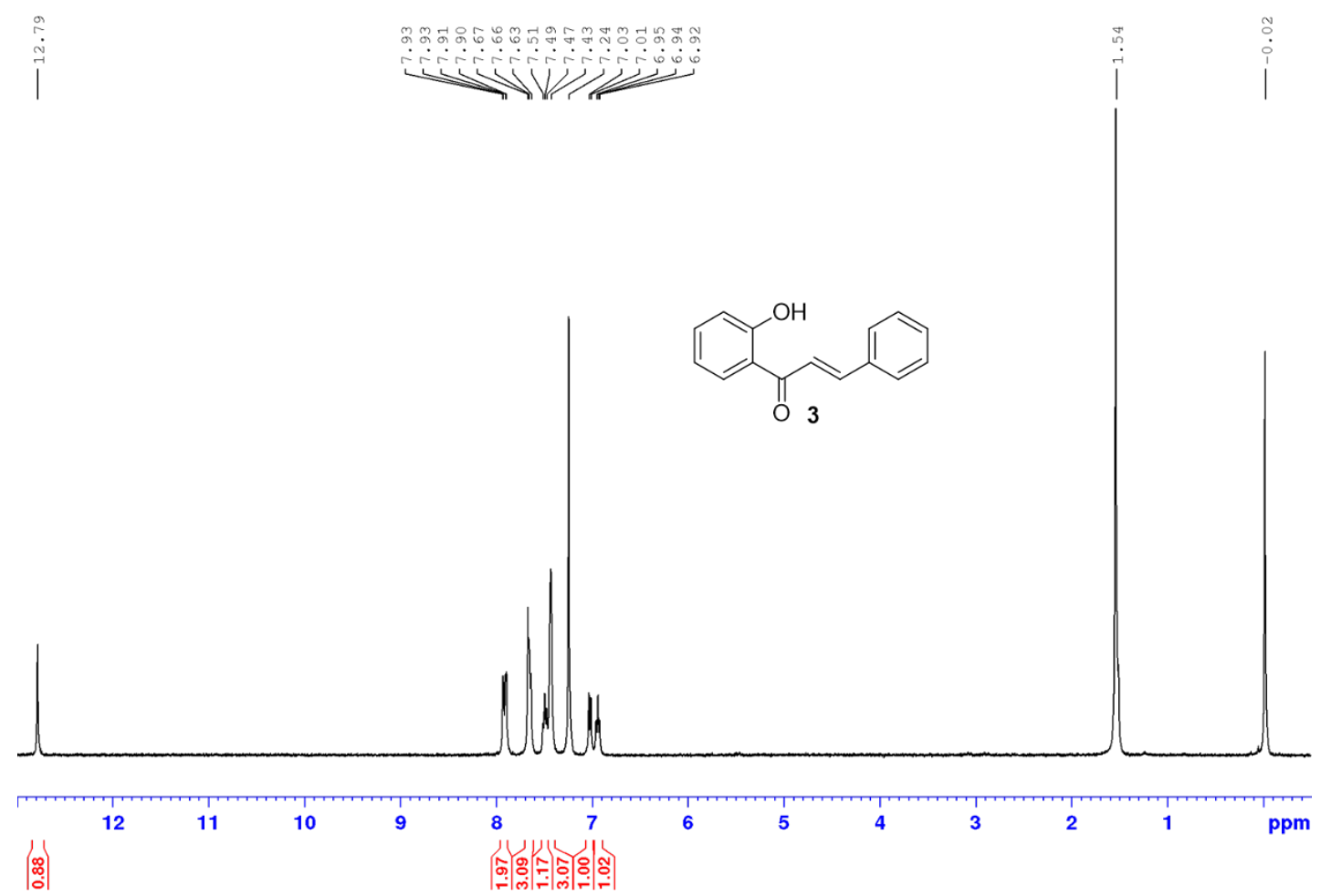

Figure S12. ${ }^{1} \mathrm{H}$ NMR of 3 in $\mathrm{CDCl}_{3}$.
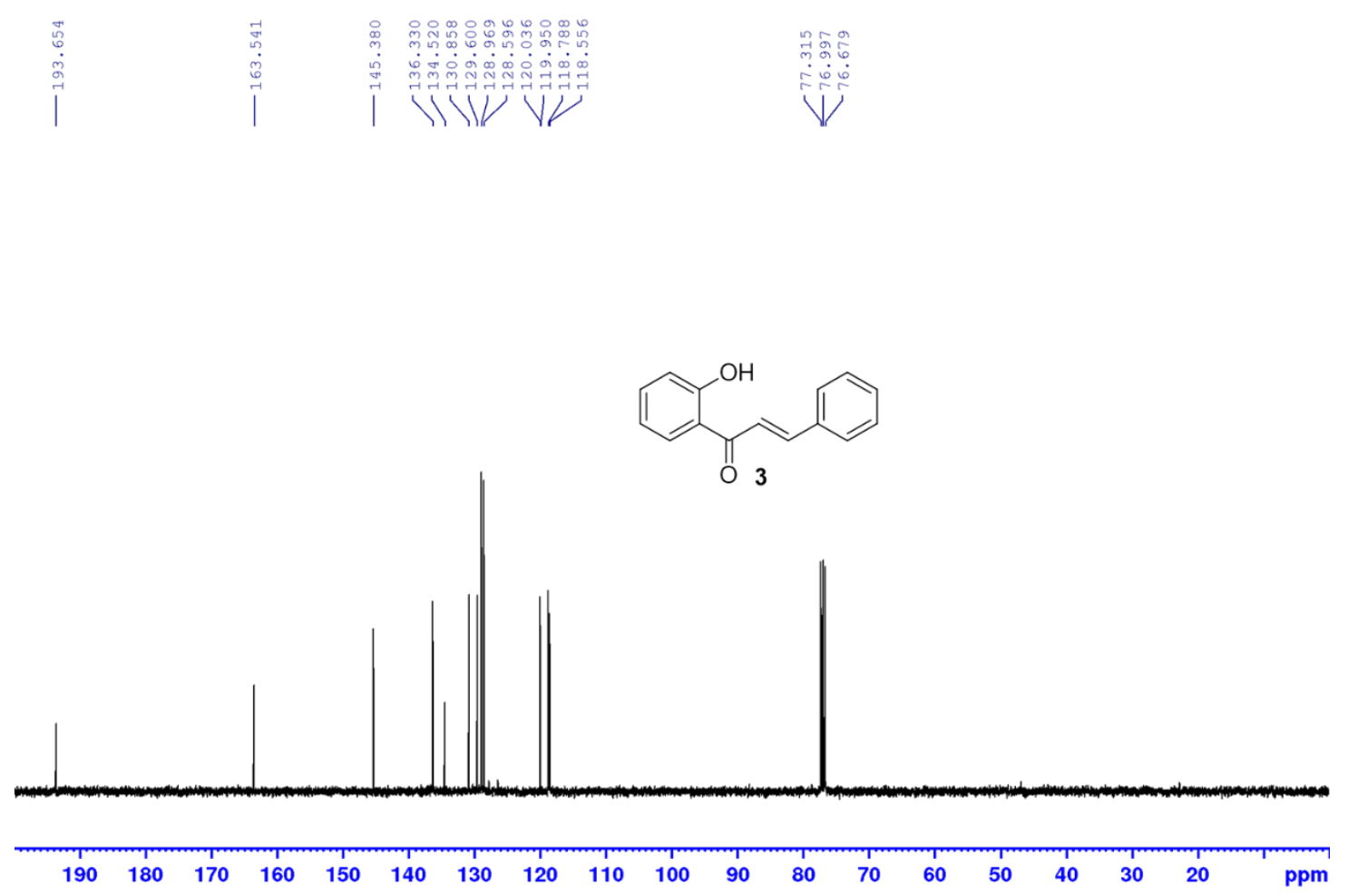

Figure S13. ${ }^{13} \mathrm{C}$ NMR of 3 in $\mathrm{CDCl}_{3}$. 


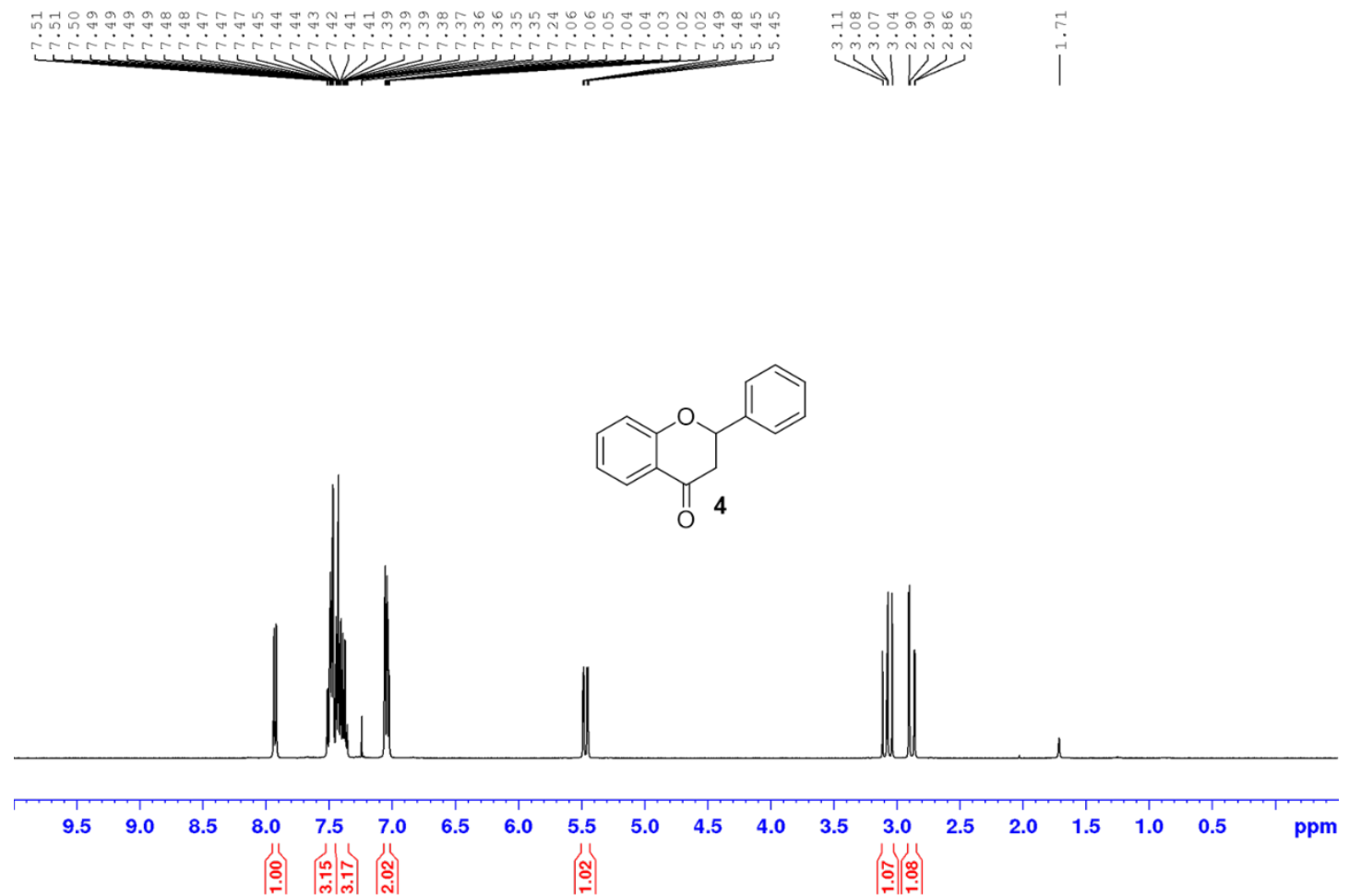

Figure S14. ${ }^{1} \mathrm{H}$ NMR of 4 in $\mathrm{CDCl}_{3}$.

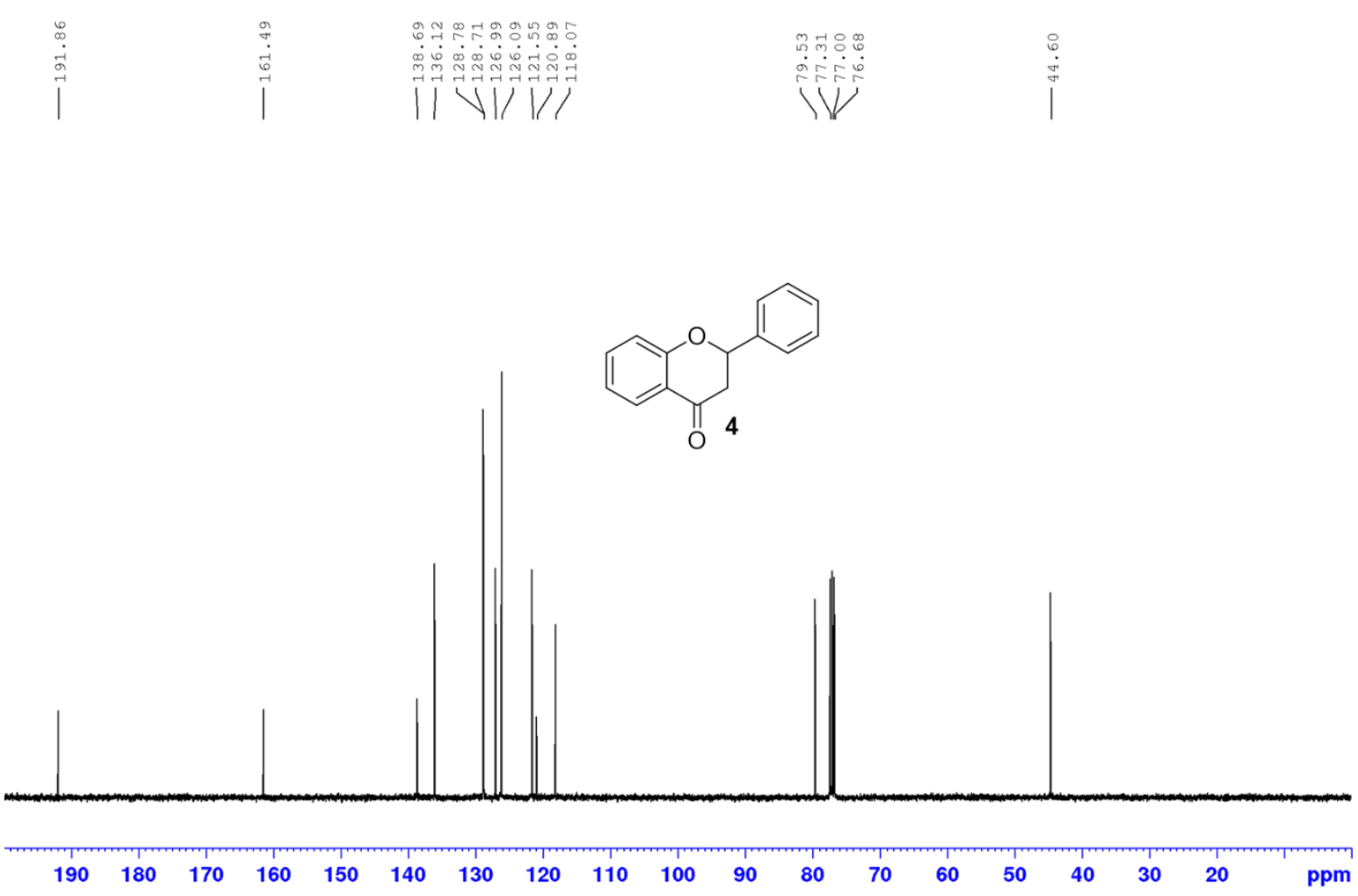

Figure S15. ${ }^{13} \mathrm{C}$ NMR of 4 in $\mathrm{CDCl}_{3}$. 

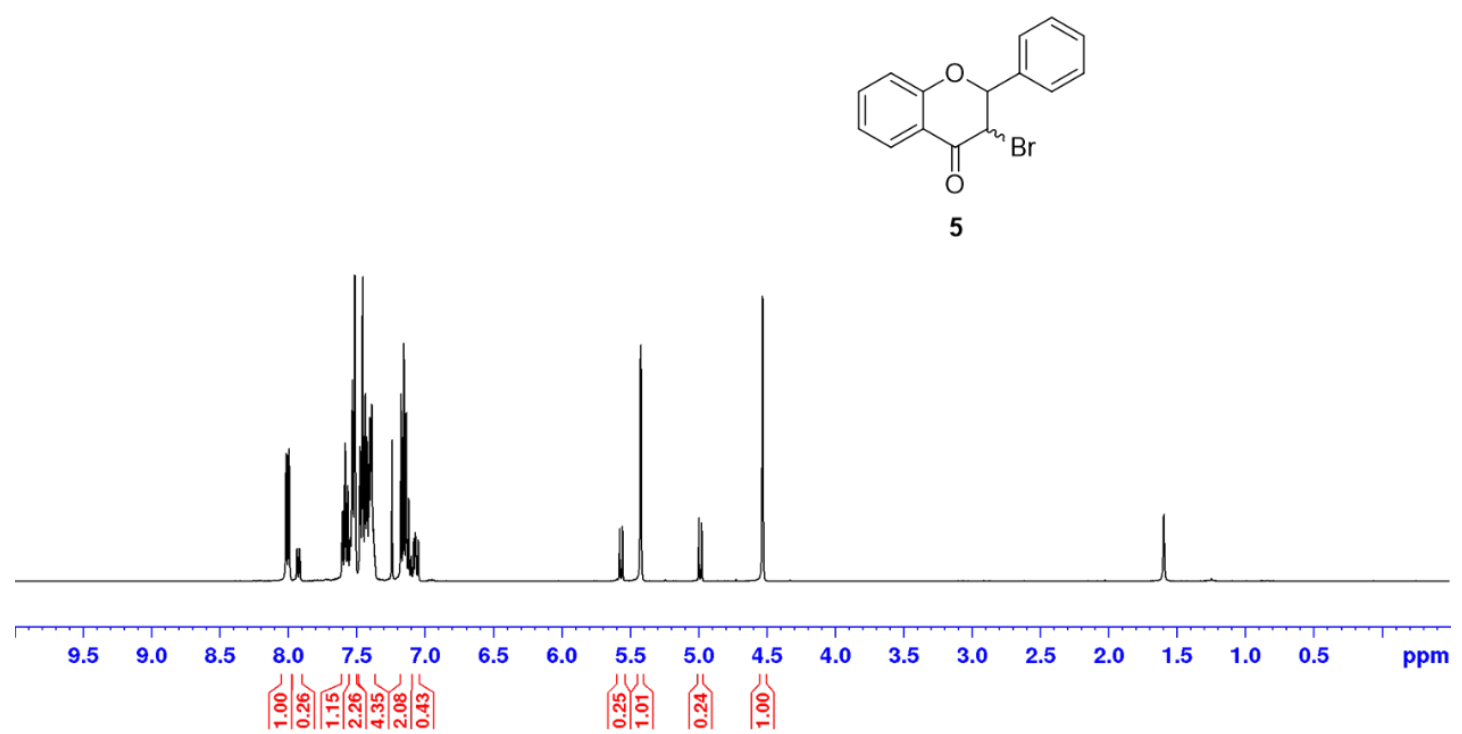

Figure S16. ${ }^{1} \mathrm{H}$ NMR of 5 in $\mathrm{CDCl}_{3}$.

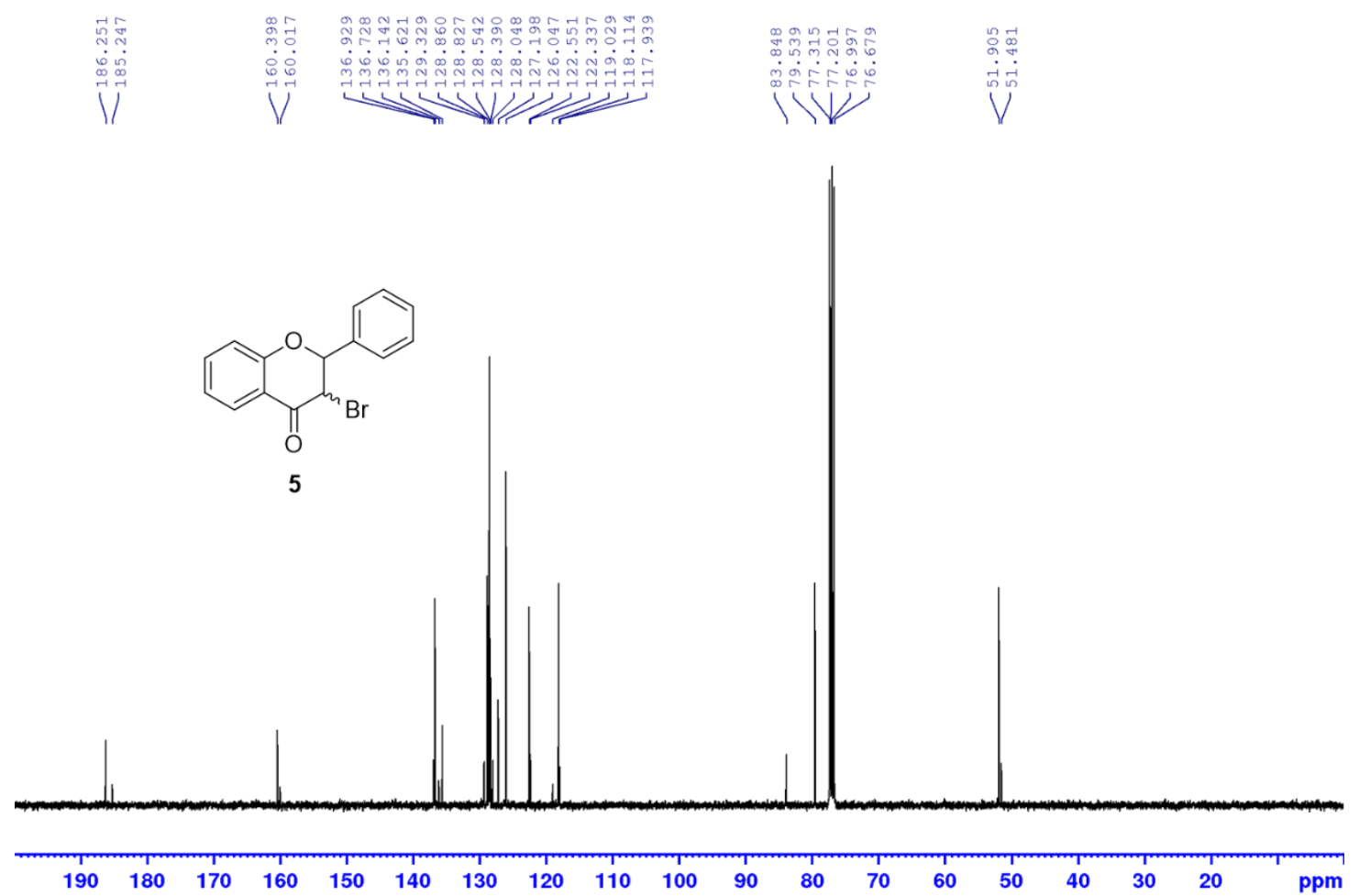

Figure S17. ${ }^{13} \mathrm{C}$ NMR of 5 in $\mathrm{CDCl}_{3}$. 


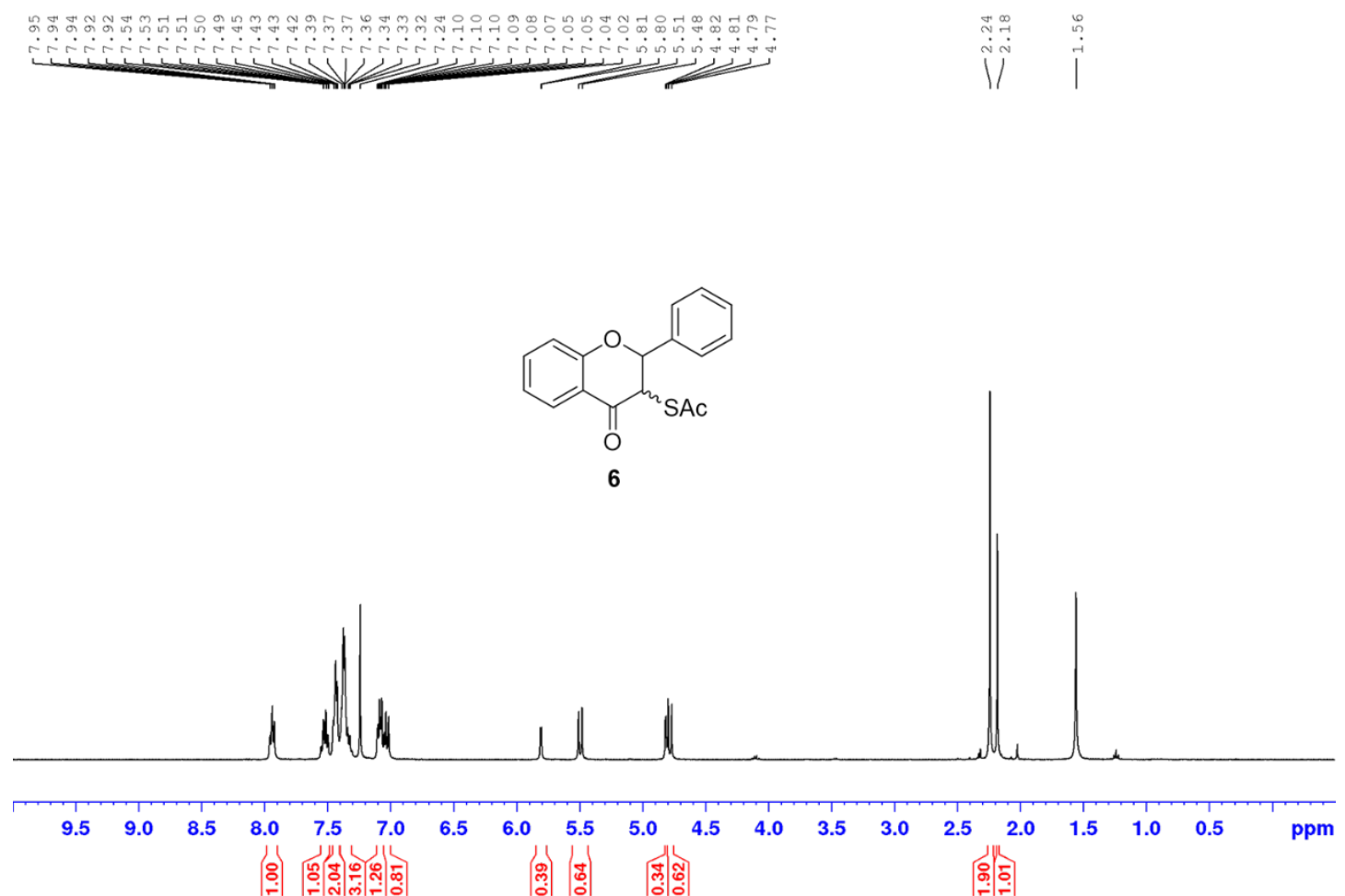

Figure S18. ${ }^{1} \mathrm{H}$ NMR of 6 in $\mathrm{CDCl}_{3}$.

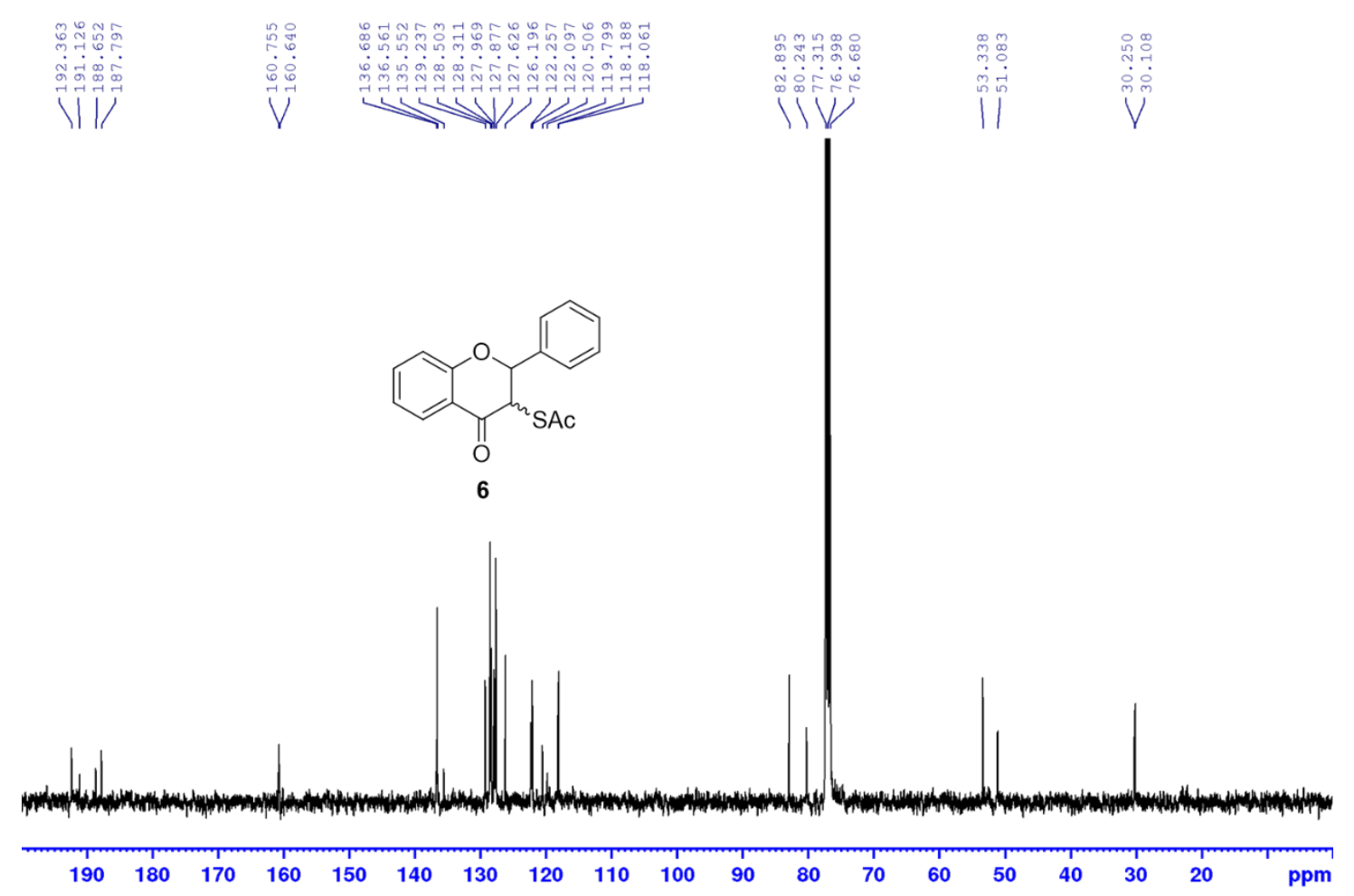

Figure S19. ${ }^{13} \mathrm{C}$ NMR of 6 in $\mathrm{CDCl}_{3}$. 


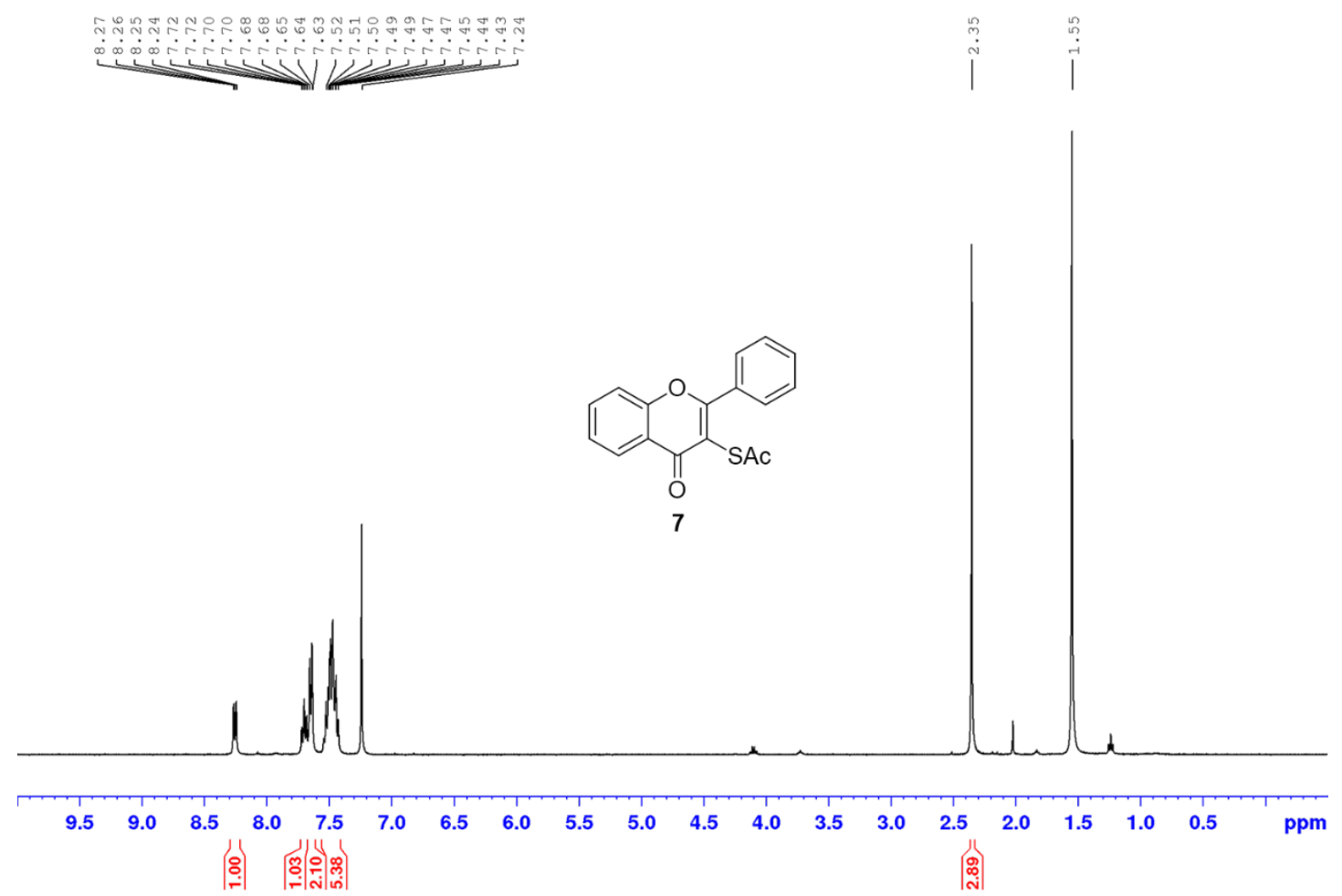

Figure S20. ${ }^{1} \mathrm{H}$ NMR of 7 in $\mathrm{CDCl}_{3}$.

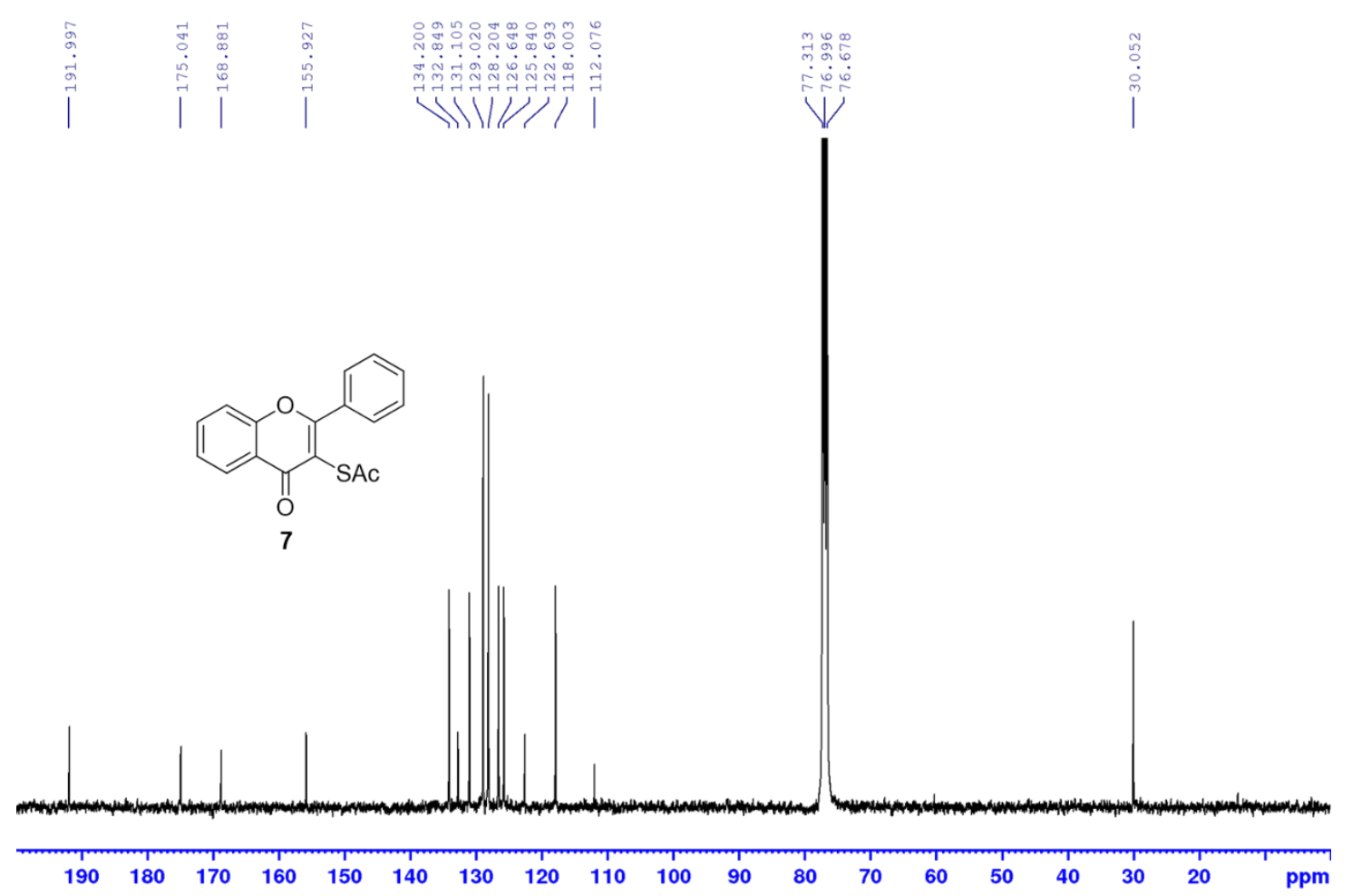

Figure S21. ${ }^{13} \mathrm{C}$ NMR of 7 in $\mathrm{CDCl}_{3}$. 


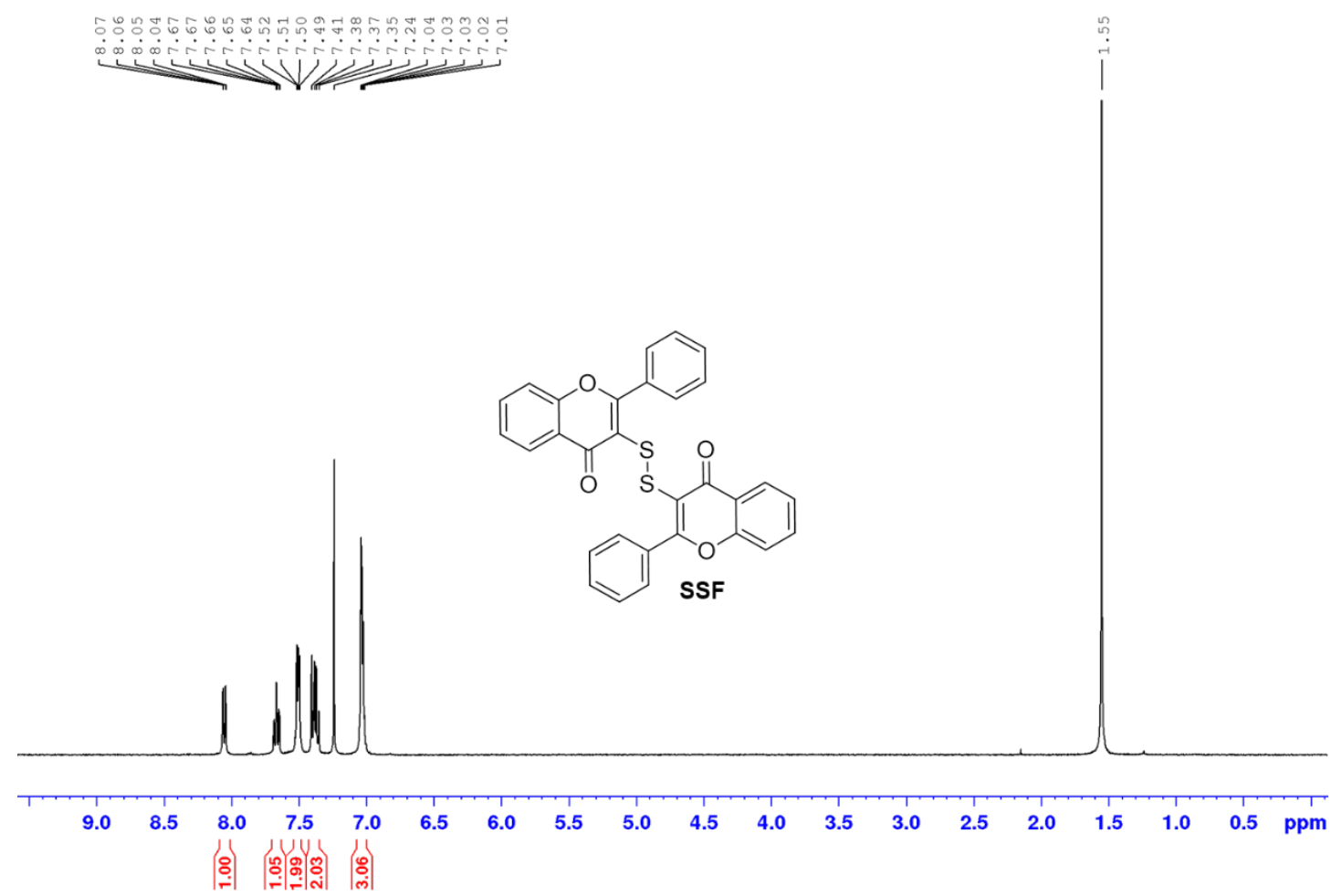

Figure S22. ${ }^{1} \mathrm{H} \mathrm{NMR}$ of $\mathbf{S S F}$ in $\mathrm{CDCl}_{3}$.

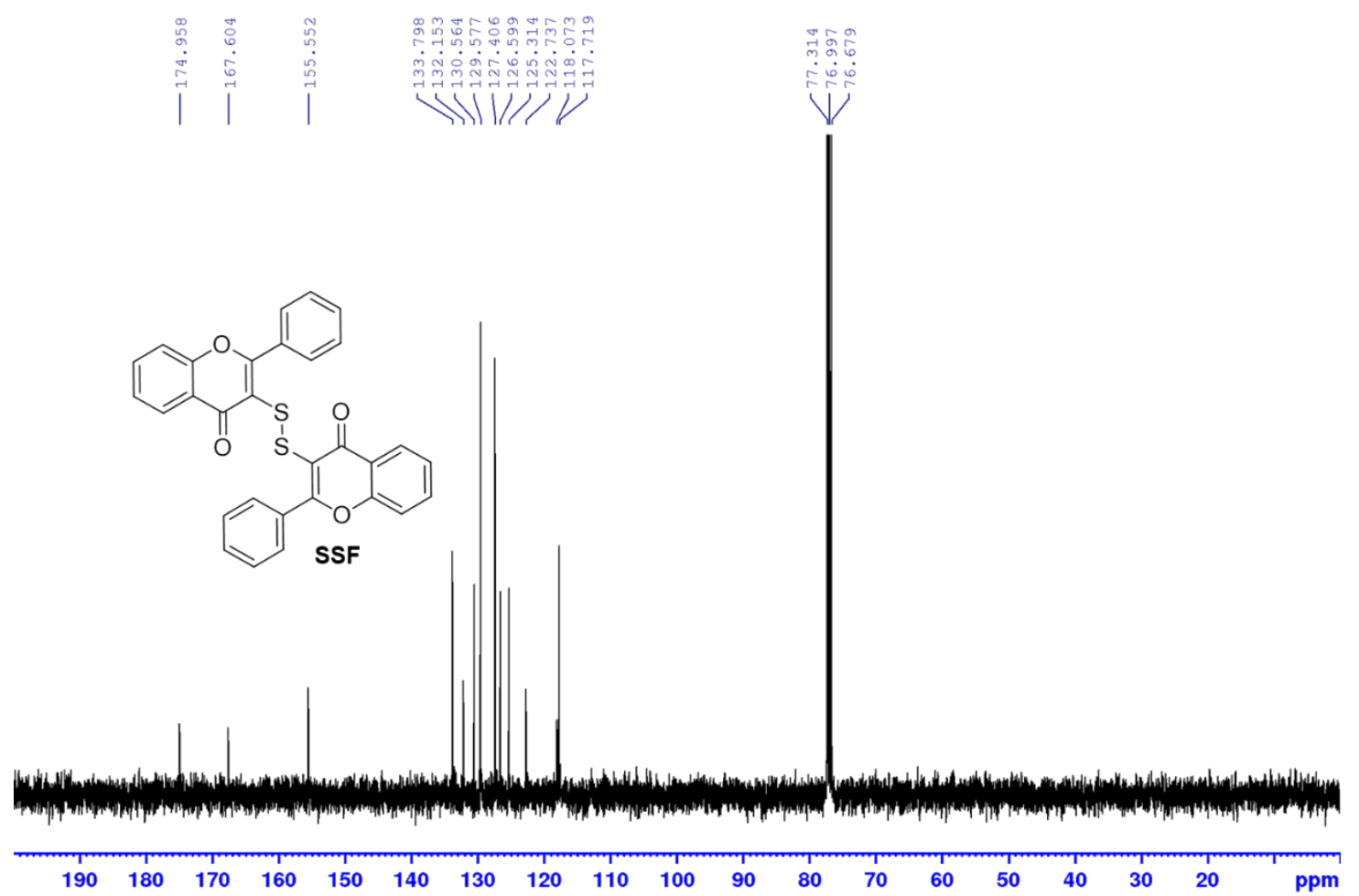

Figure S23. ${ }^{13} \mathrm{C}$ NMR of SSF in $\mathrm{CDCl}_{3}$. 


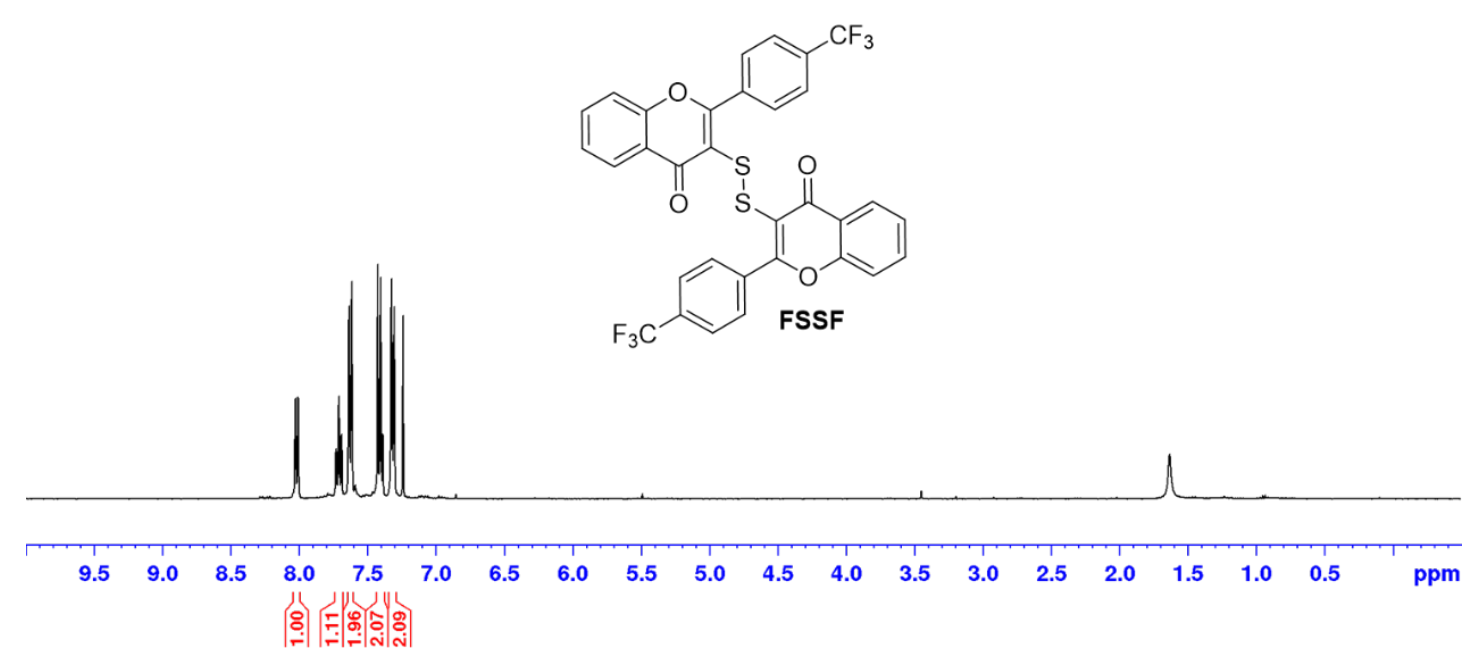

Figure S24. ${ }^{1} \mathrm{H}$ NMR of FSSF in $\mathrm{CDCl}_{3}$.

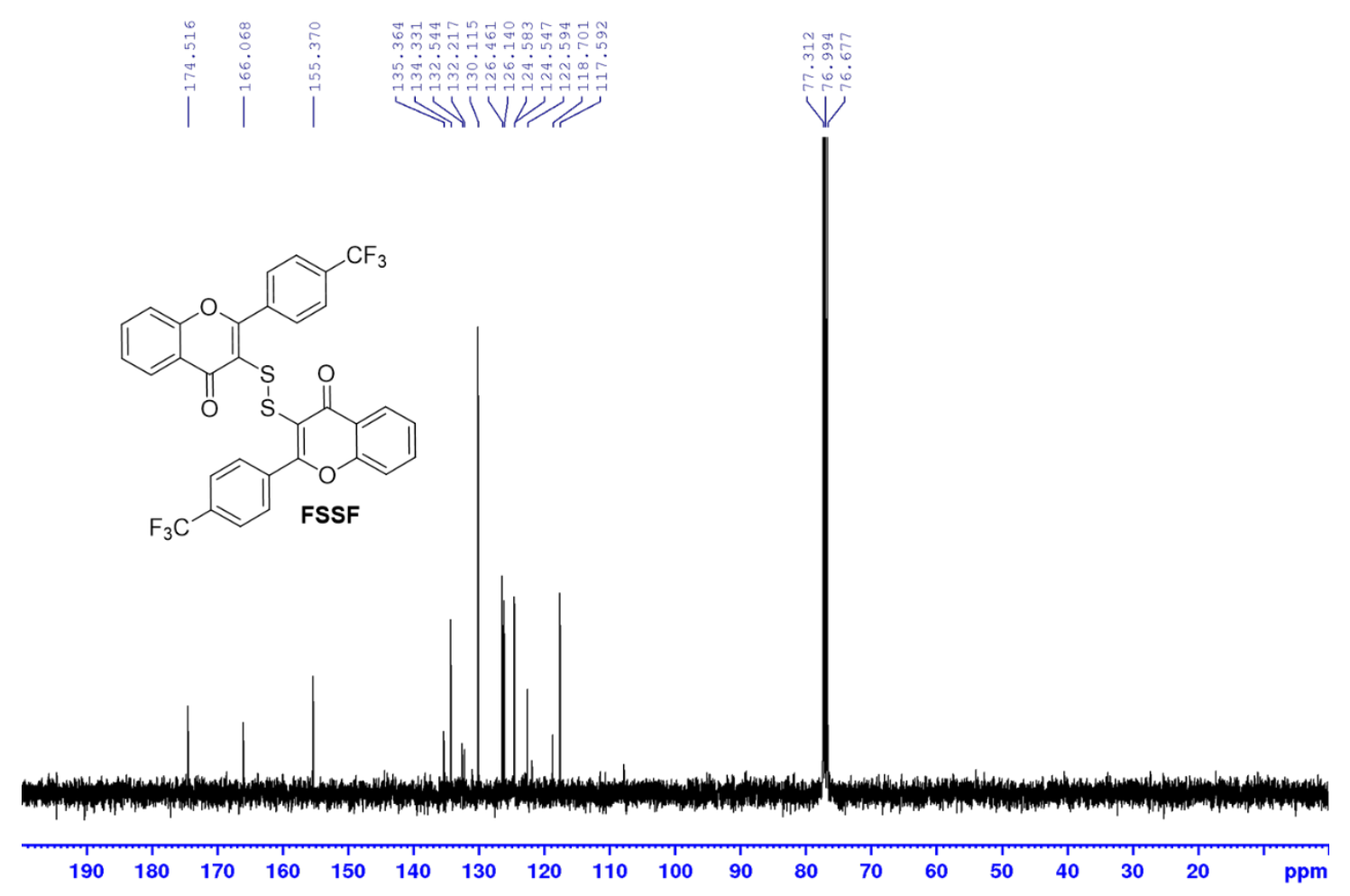

Figure S25. ${ }^{13} \mathrm{C}$ NMR of FSSF in $\mathrm{CDCl}_{3}$. 


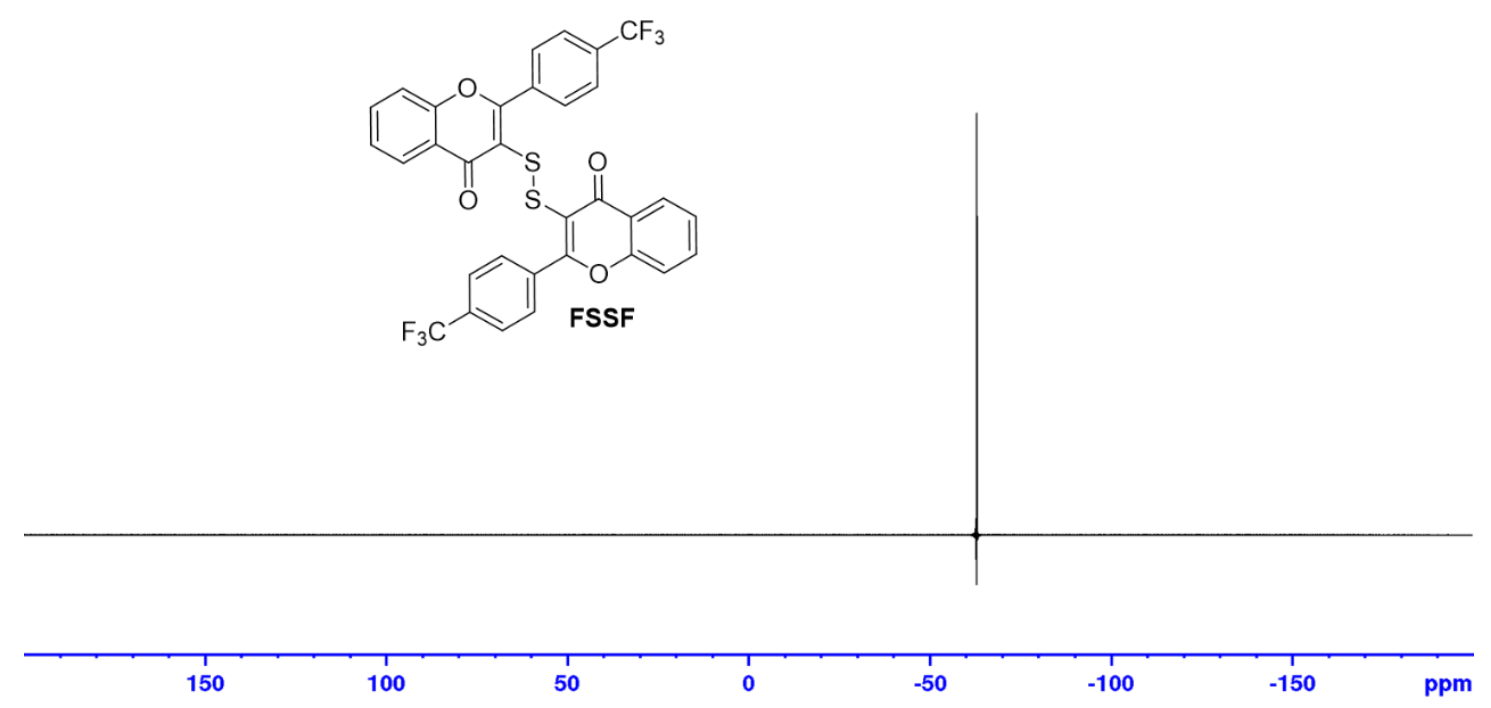

Figure S26. ${ }^{19} \mathrm{~F}$ NMR of FSSF in $\mathrm{CDCl}_{3}$. 


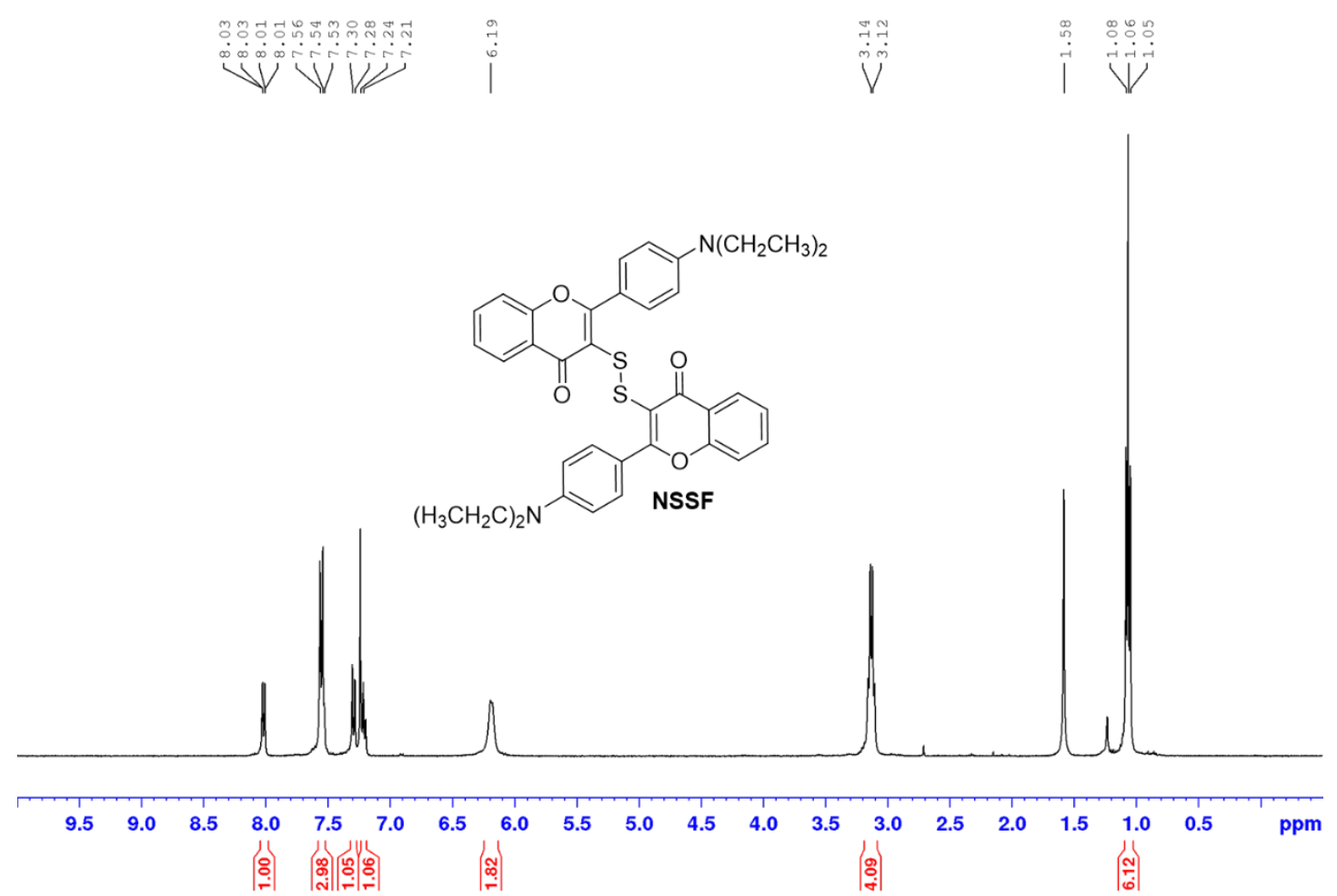

Figure S27. ${ }^{1} \mathrm{H}$ NMR of NSSF in $\mathrm{CDCl}_{3}$.

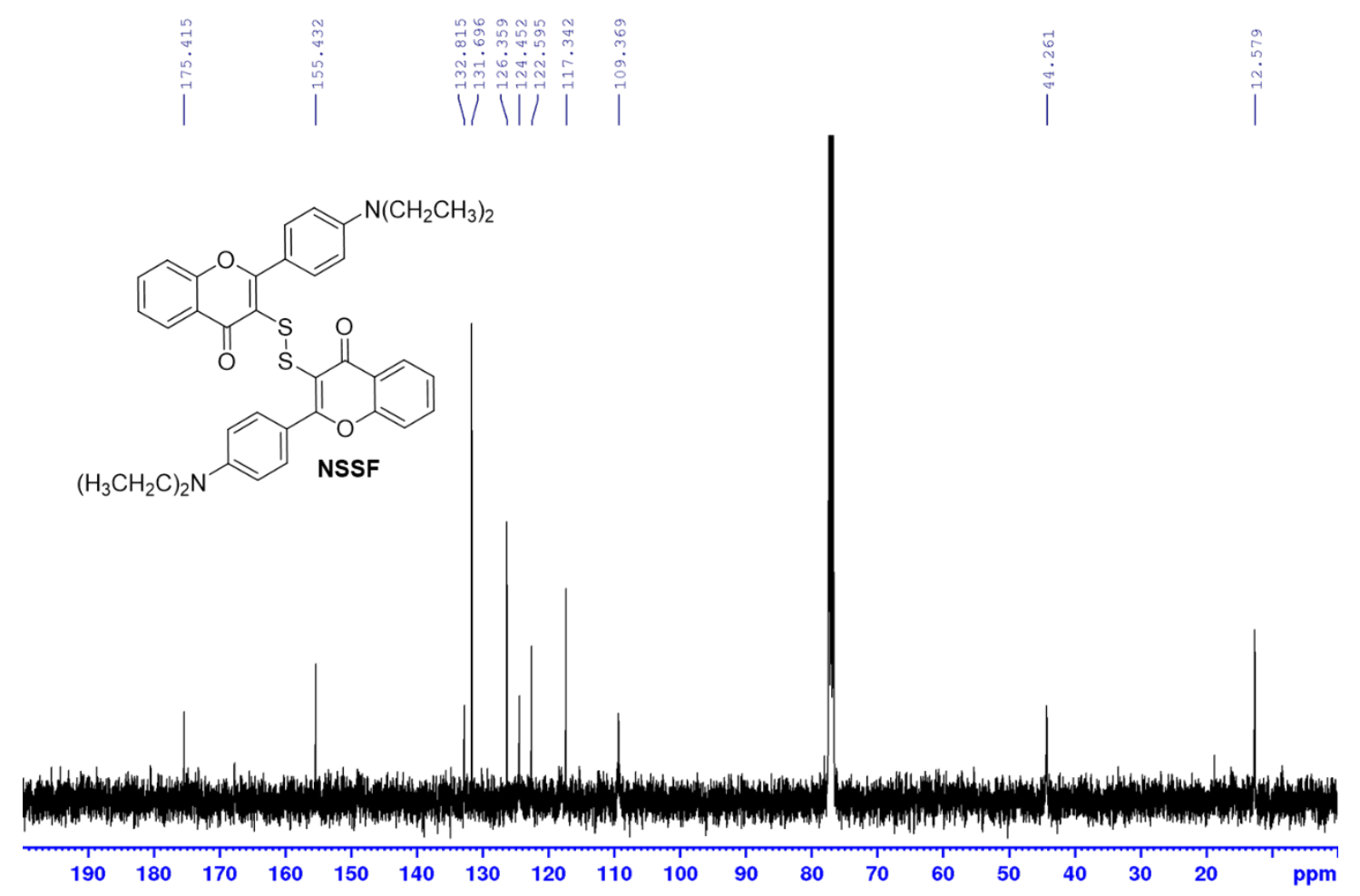

Figure S28. ${ }^{13} \mathrm{C}$ NMR of NSSF in $\mathrm{CDCl}_{3}$. 


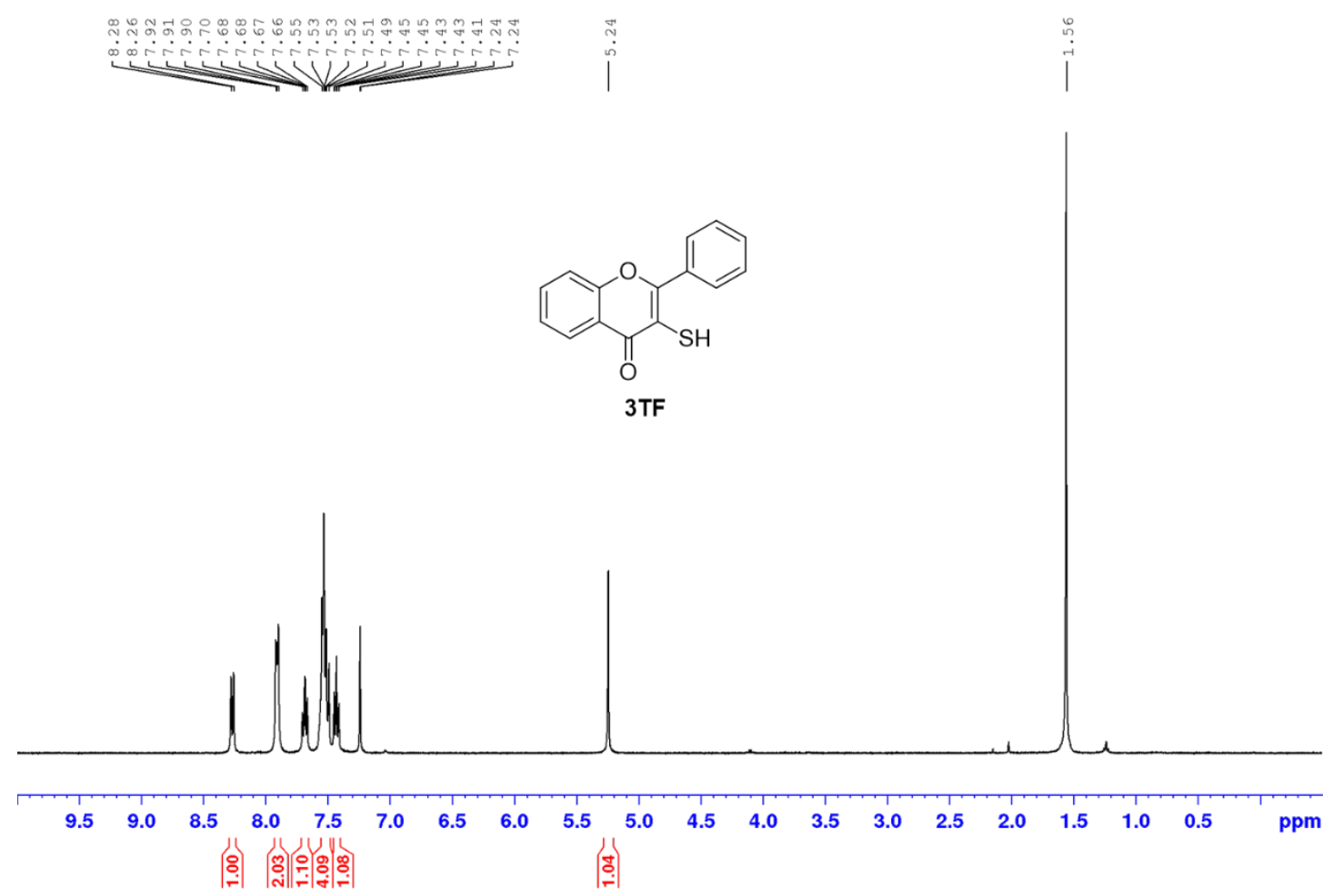

Figure S29. ${ }^{1} \mathrm{H} \mathrm{NMR}$ of $\mathbf{3 T F}$ in $\mathrm{CDCl}_{3}$.

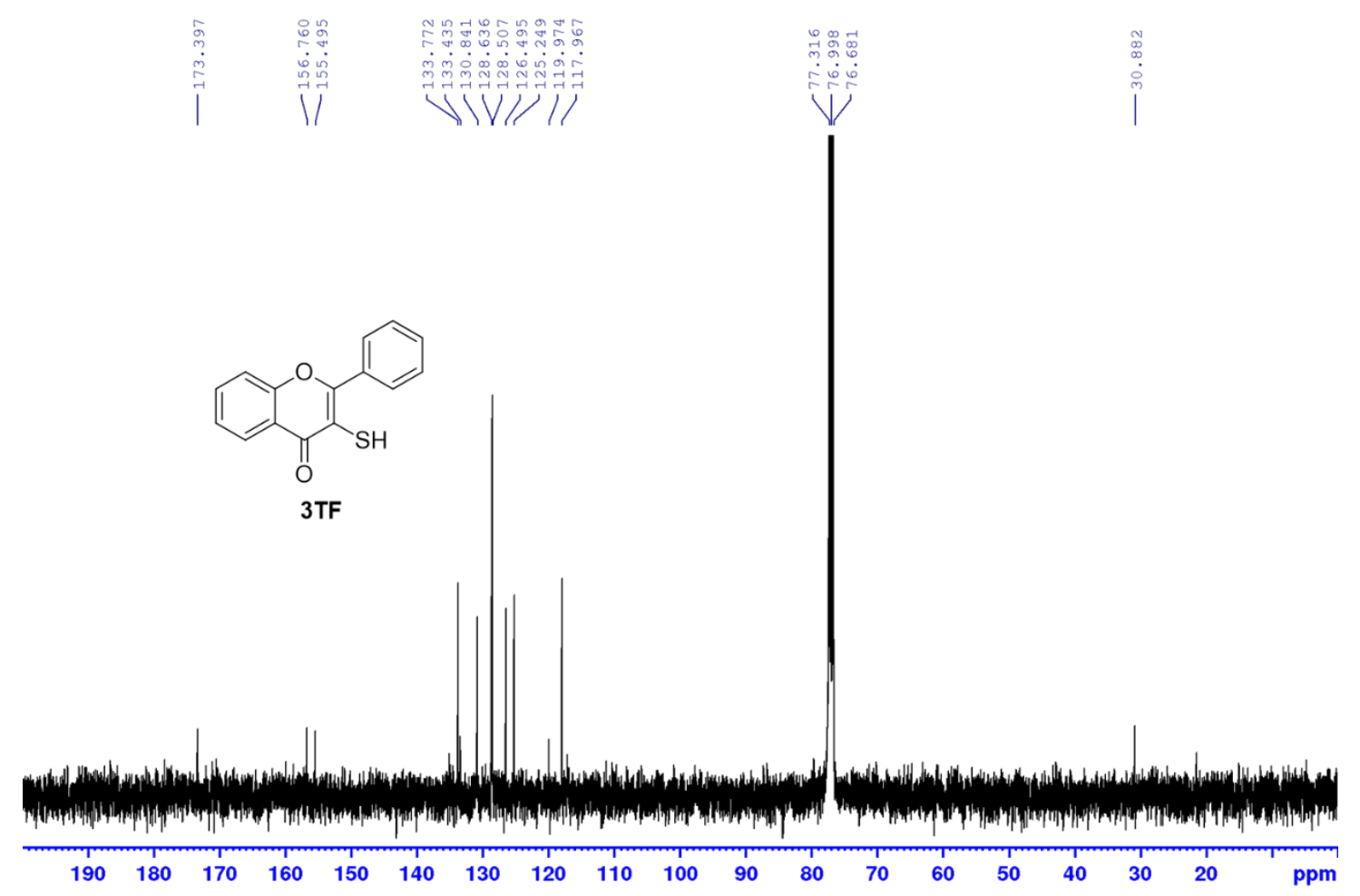

Figure S30. ${ }^{13} \mathrm{C} \mathrm{NMR}$ of $\mathbf{3 T F}$ in $\mathrm{CDCl}_{3}$. 

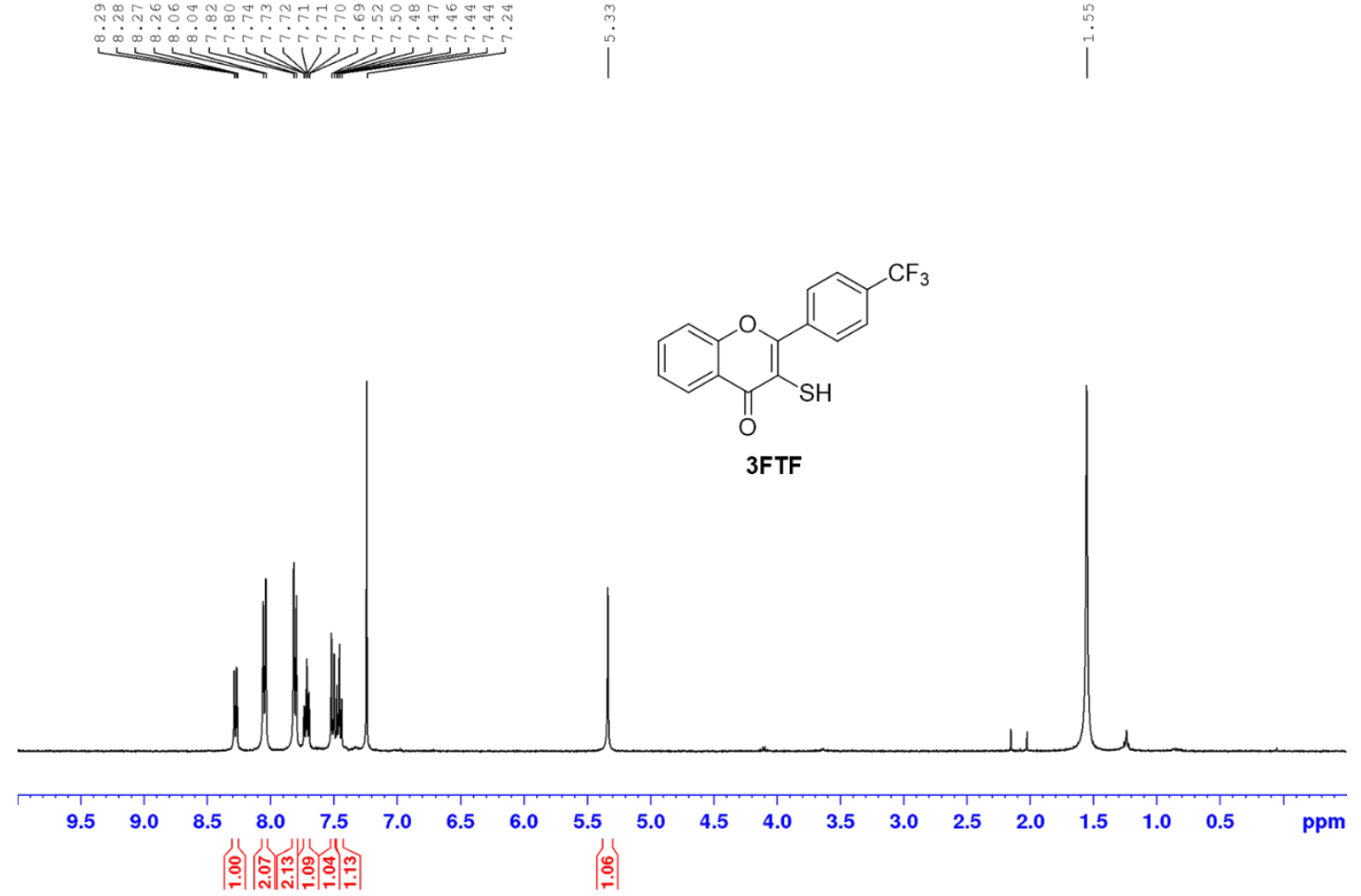

Figure S31. ${ }^{1} \mathrm{H}$ NMR of $\mathbf{3 F T F}$ in $\mathrm{CDCl}_{3}$.

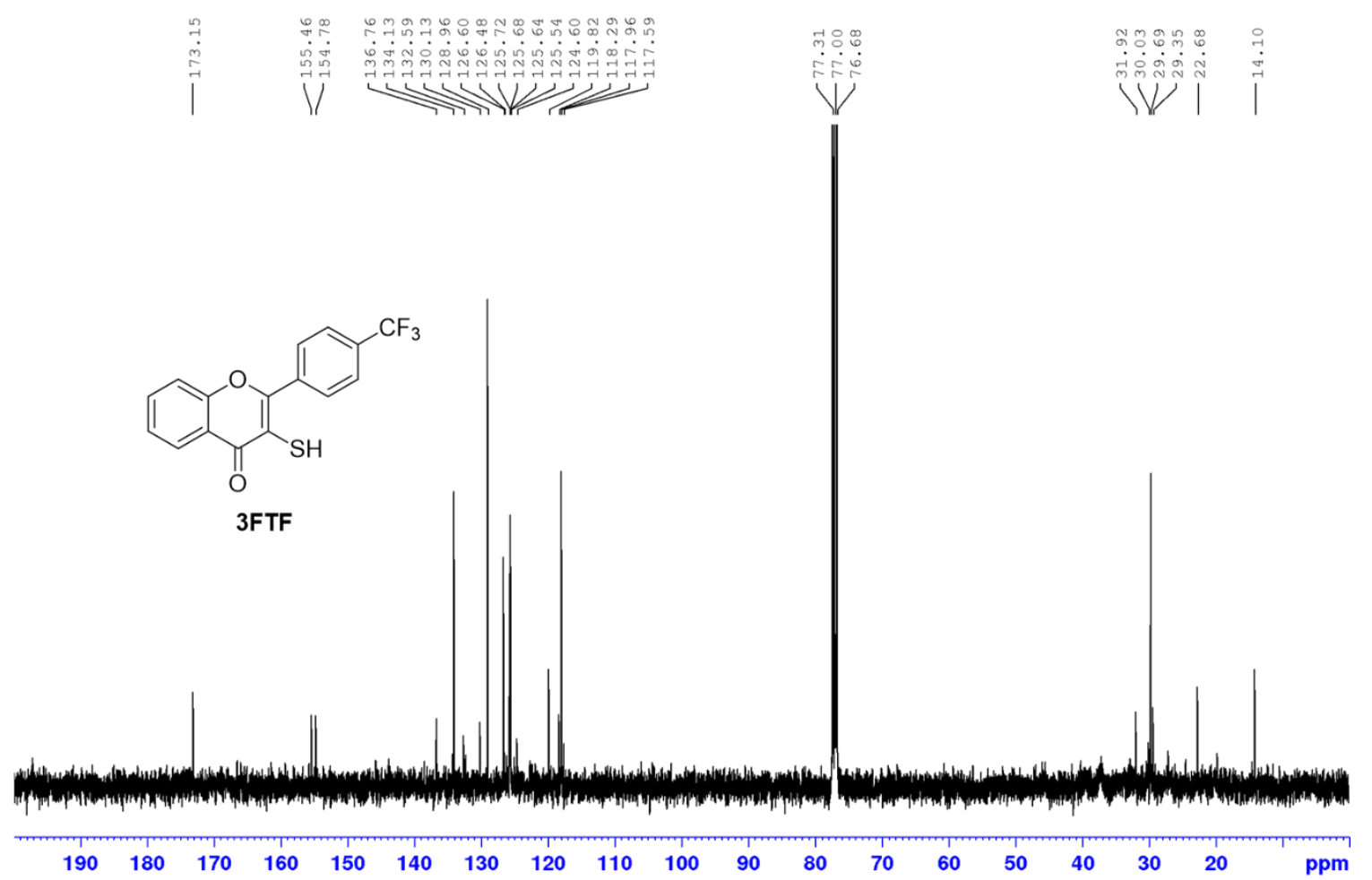

Figure S32. ${ }^{13} \mathrm{C}$ NMR of $\mathbf{3 F T F}$ in $\mathrm{CDCl}_{3}$. 


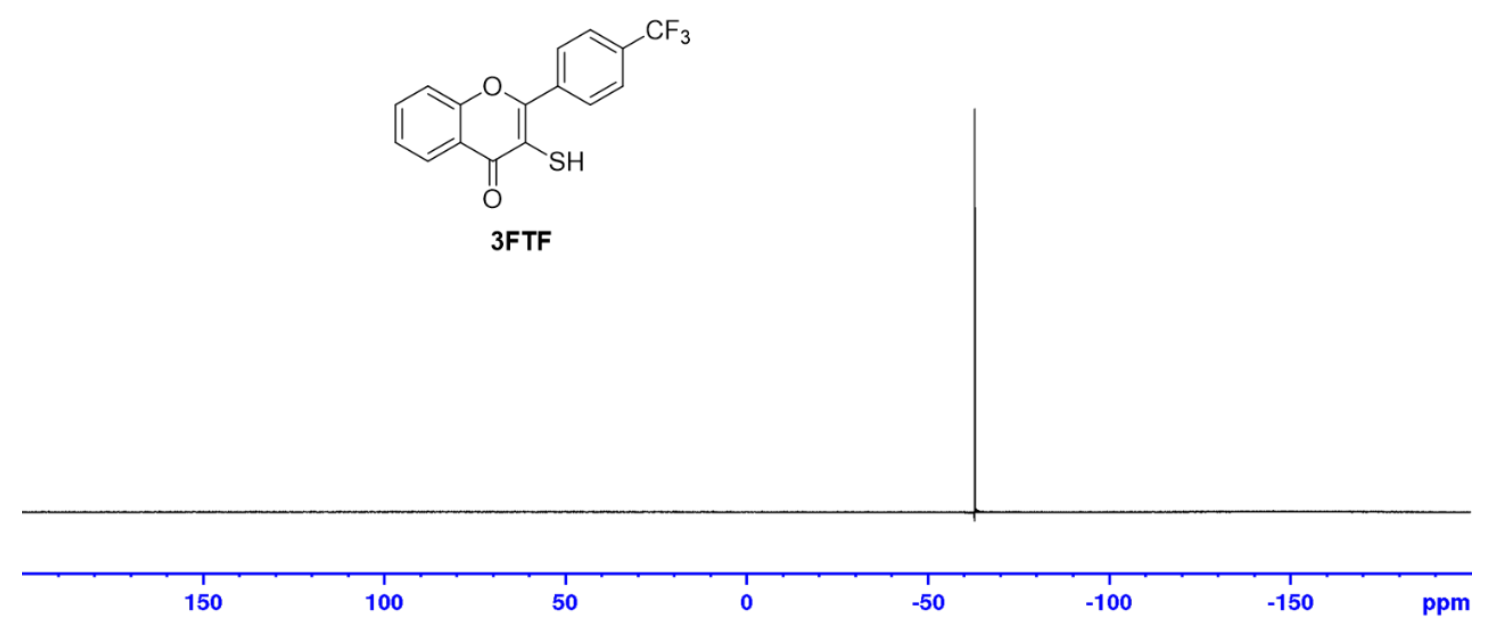

Figure S33. ${ }^{19} \mathrm{~F}$ NMR of 3FTF in $\mathrm{CDCl}_{3}$. 


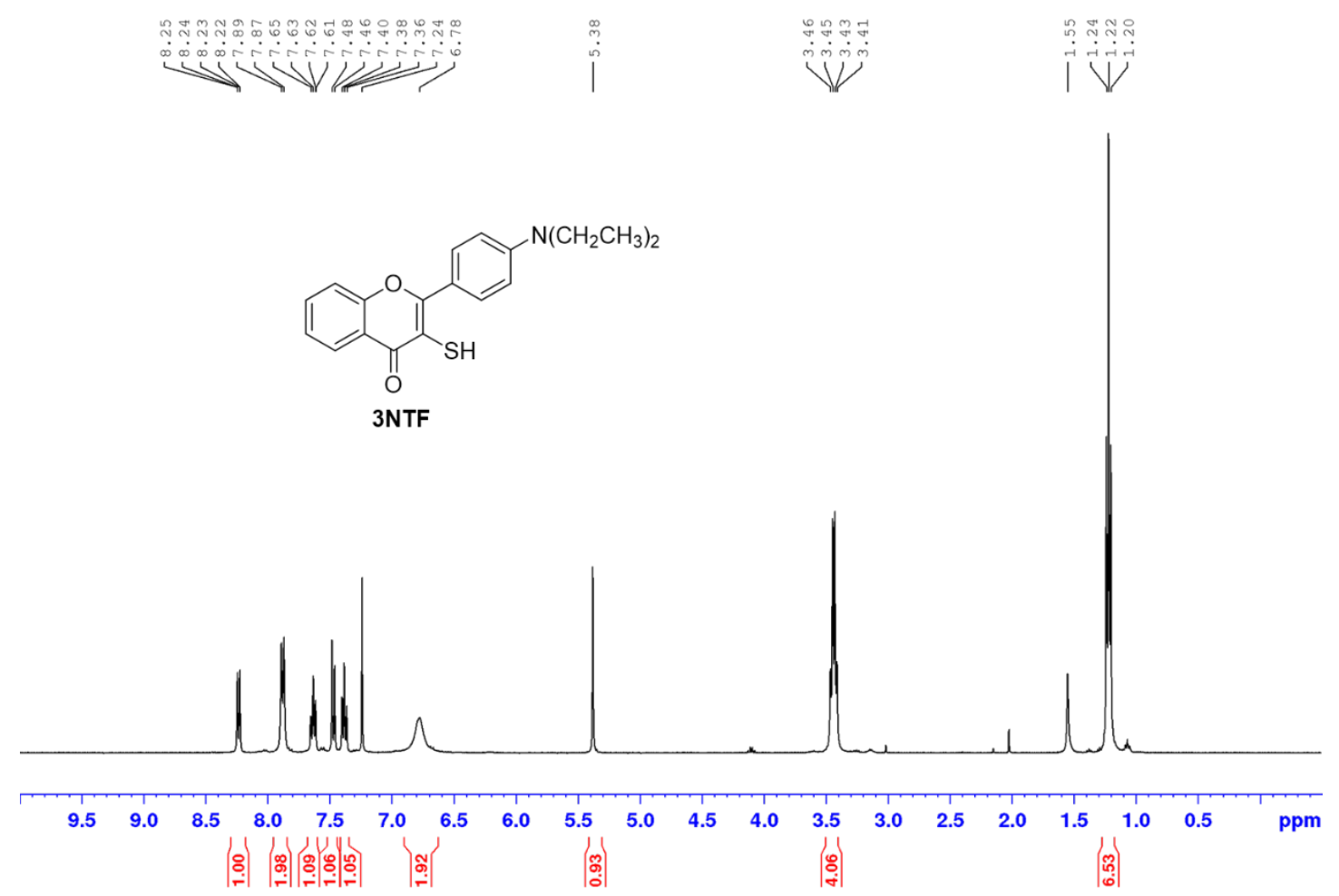

Figure S34. ${ }^{1} \mathrm{H}$ NMR of $\mathbf{3 N T F}$ in $\mathrm{CDCl}_{3}$.

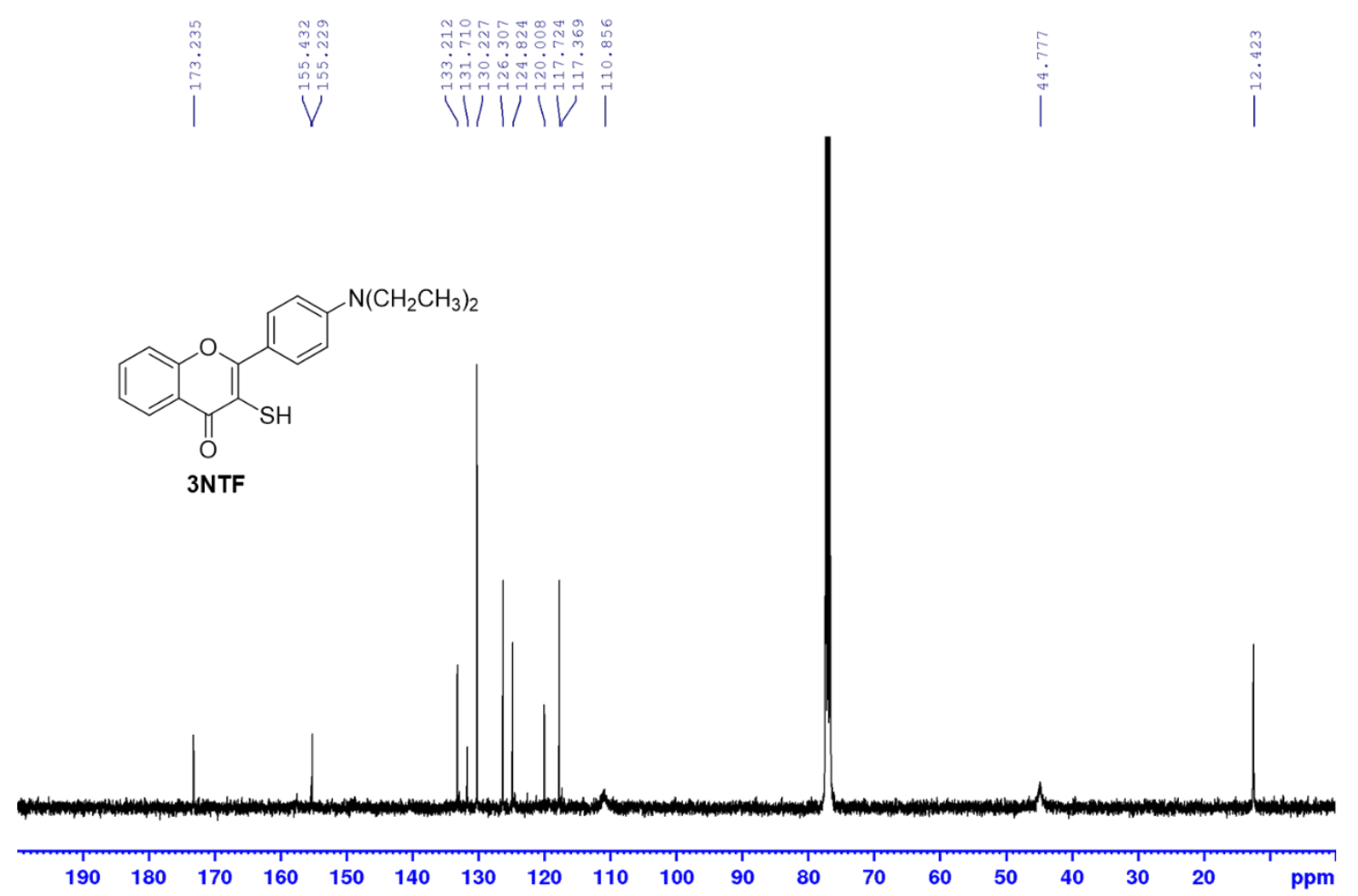

Figure S35. ${ }^{13} \mathrm{C}$ NMR of $\mathbf{3 N T F}$ in $\mathrm{CDCl}_{3}$. 


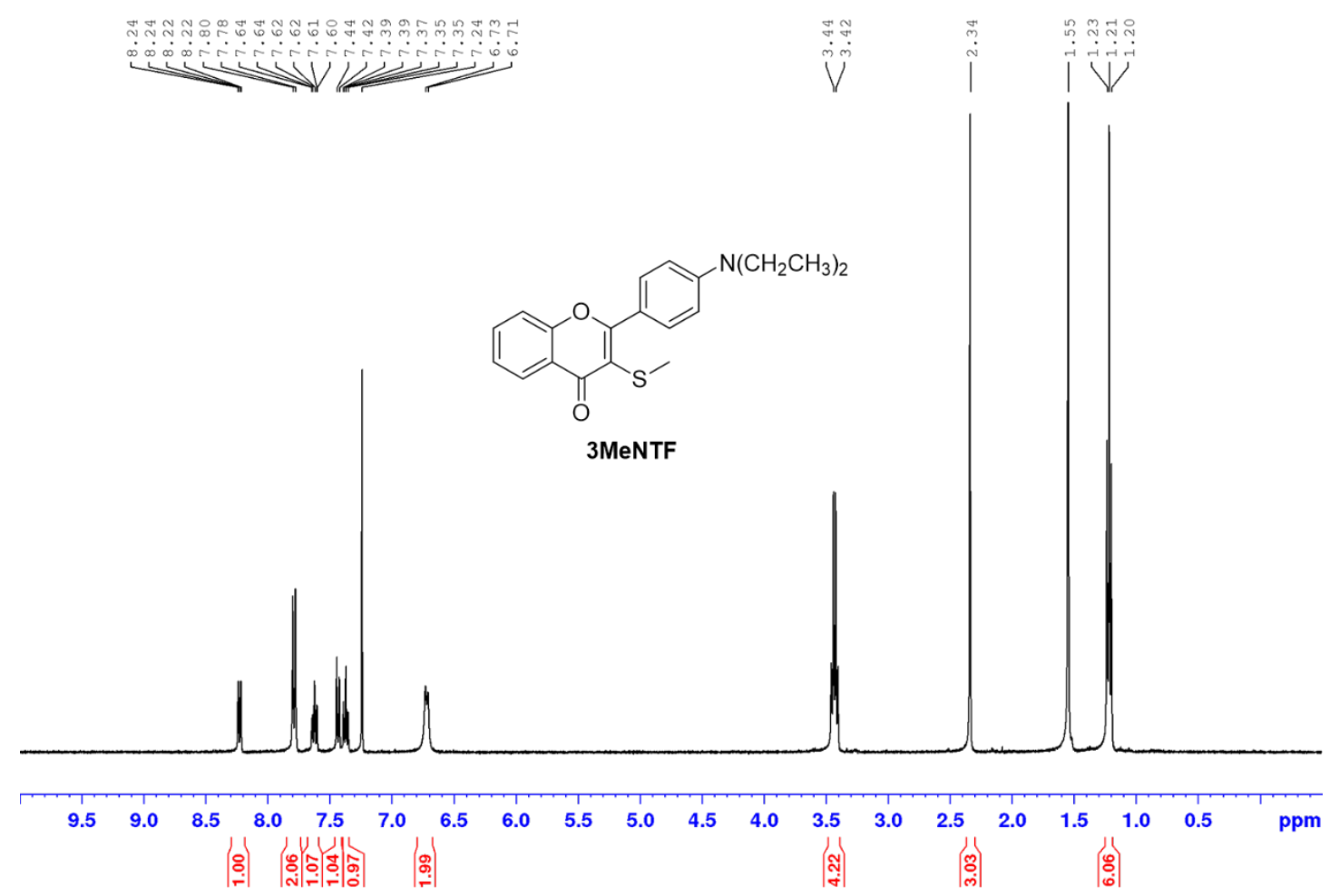

Figure S36. ${ }^{1} \mathrm{H}$ NMR of $\mathbf{3 M e N T F}$ in $\mathrm{CDCl}_{3}$.

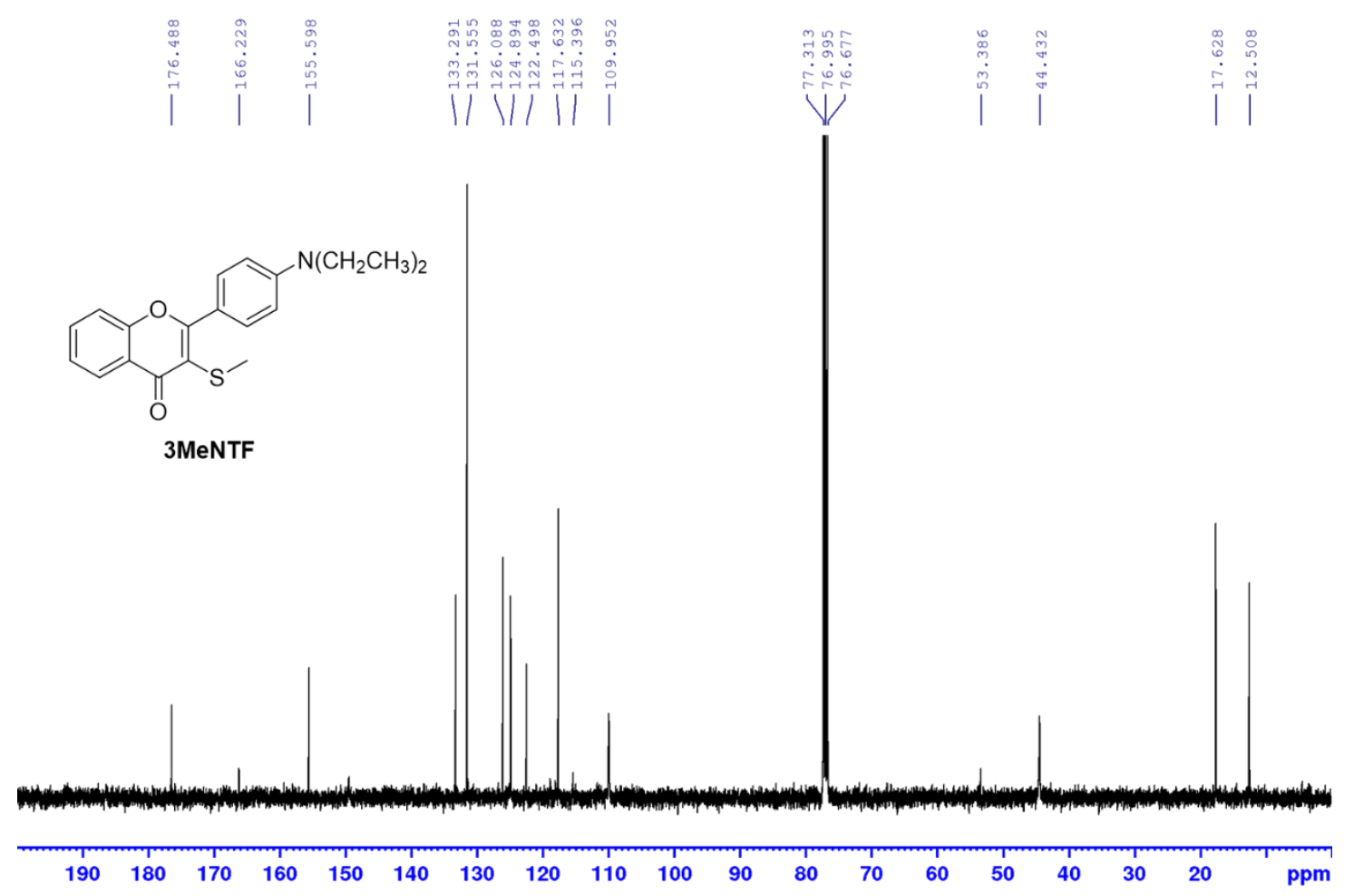

Figure S37. ${ }^{13} \mathrm{C}$ NMR of $\mathbf{3 M e N T F}$ in $\mathrm{CDCl}_{3}$. 


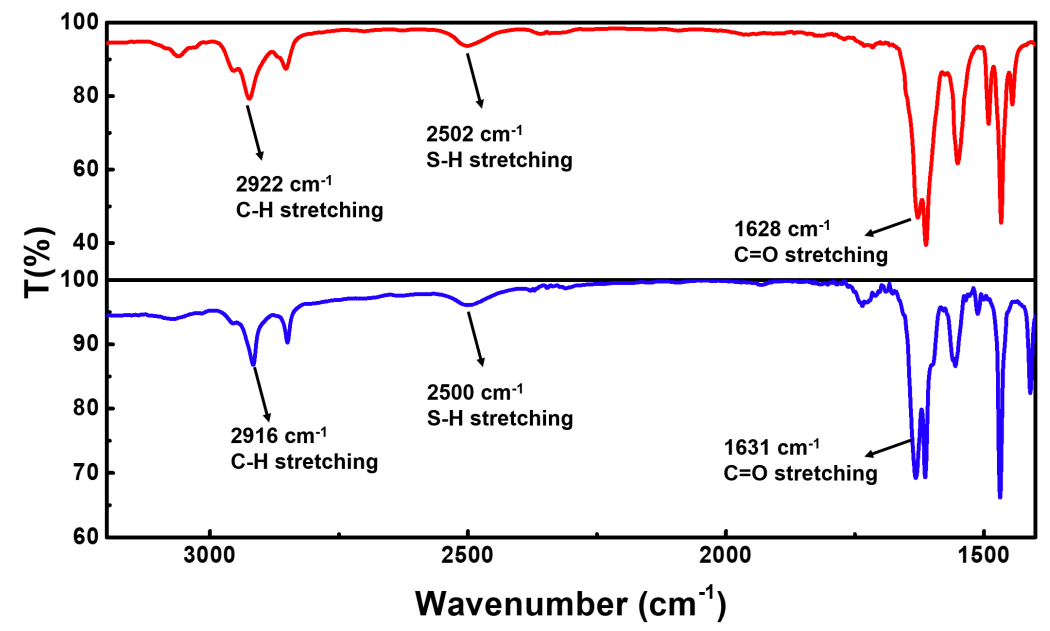

Figure S38. The mid-IR spectra of 3TF (top) and 3FTF (bottom).

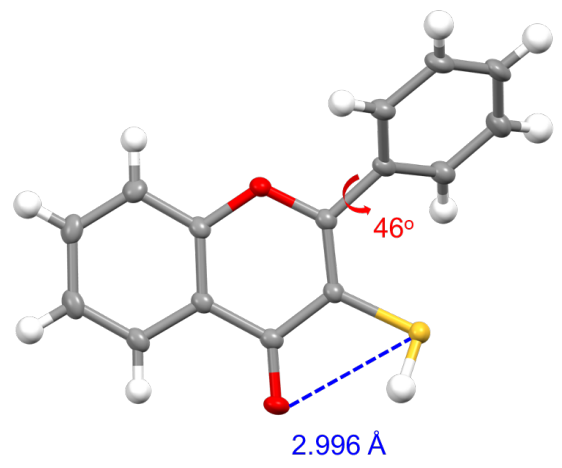

Figure S39. The X-ray crystallographic structure of 3TF. The displacement ellipsoids are drawn at the $50 \%$ probability level, and the $\mathrm{H}$ atoms are drawn as spheres of arbitrary radii.

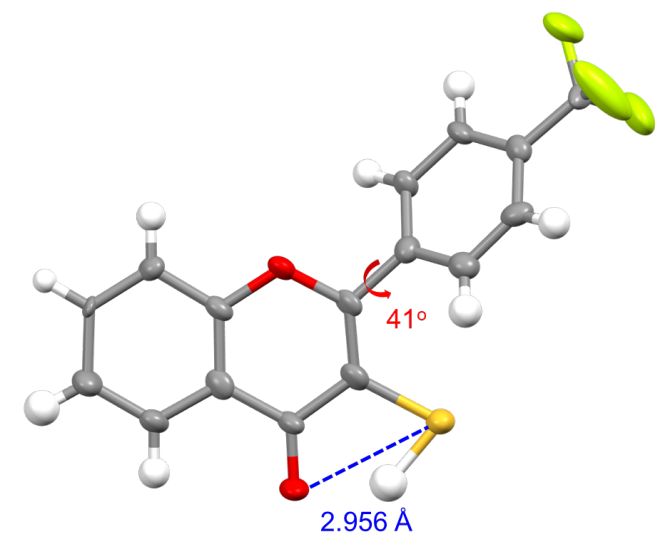

Figure S40. The X-ray crystallographic structure of 3FTF. The displacement ellipsoids are drawn at the $50 \%$ probability level, and the $\mathrm{H}$ atoms are drawn as spheres of arbitrary radii. 

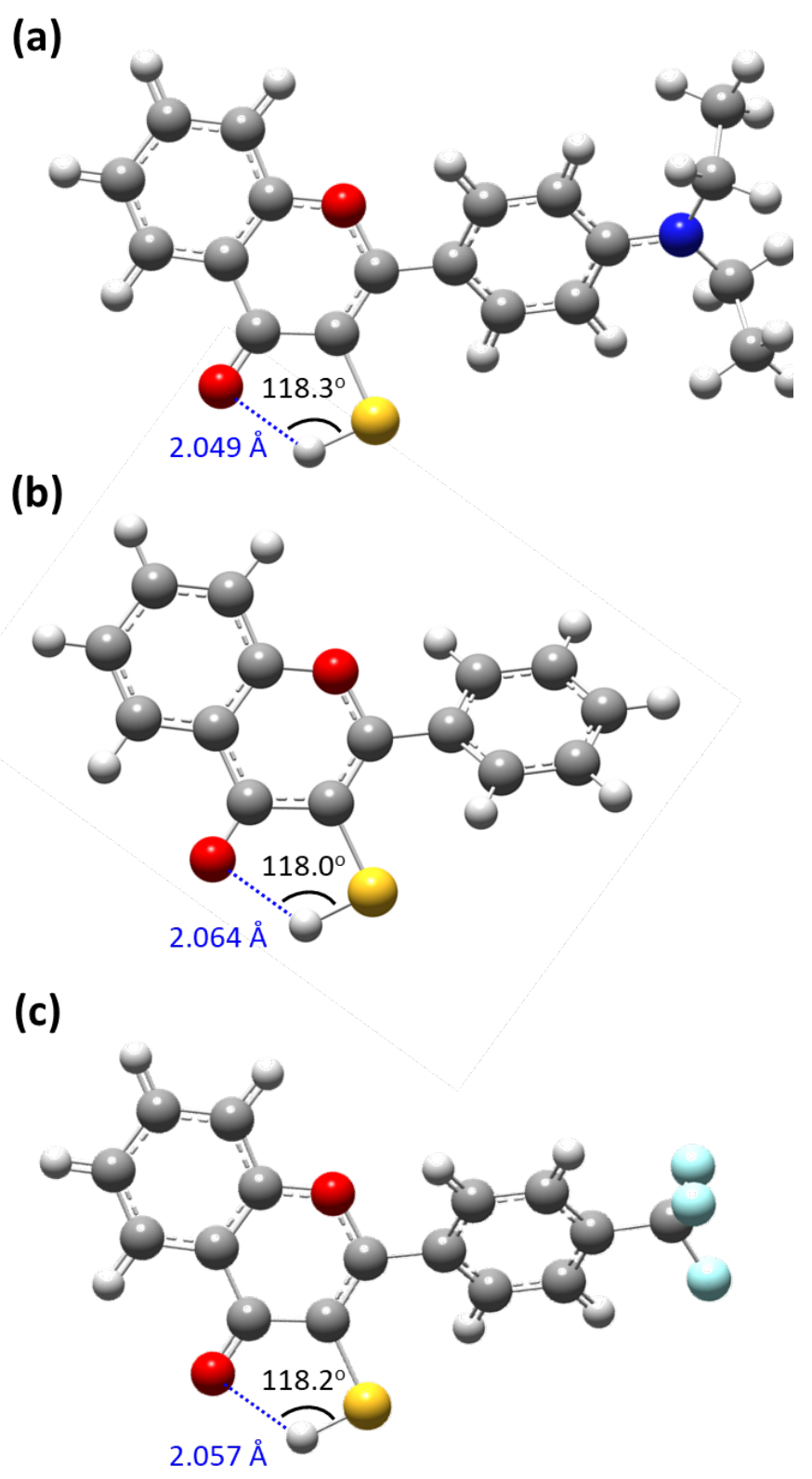

Figure S41. The computed ground state geometries for (a) 3NTF, (b) 3TF and (c) 3FTF at the B3LYP/6-311++G(3df,3pd) level. The hydrogen bond distance was denoted by blue dashed line. 


\section{Photophysical Properties}

Steady-state spectroscopy. The UV-Vis absorption spectra and the emission spectra were performed by a U-3310 spectrophotometer (Hitachi) and a FLS980 fluorometer (Edinburgh Instrument), respectively. Both excitation and emission wavelength of FLS 980 were carefully calibrated. The samples were prepared in a $1-\mathrm{cm}$ length cuvette with the absorbance of $0.3\left(\mathrm{ca} .2 \times 10^{-5} \mathrm{M}\right)$ at the excitation wavelength.

Time-resolved fluorescence spectroscopy. The sub-nanosecond time-resolved spectra were recorded by a time-correlated single photon counting (TCSPC) system (OB-900, Edinburgh Instrument). The excitation light source was set up by second harmonic generation (SHG) of a pulse-selected tsunami femtosecond laser pulses of $780 \mathrm{~nm}$ and generating $390 \mathrm{~nm}$ light. The fluorescence of the sample was collected at an angle of $90^{\circ}$ with respect to the pump beam. A polarizer which is set at $54.7^{\circ}$ with respect to the polarization of the pump beam was located on the light path of the pump beam in front of the detector to eliminate the anisotropy. The temporal resolution was estimated to be $15 \mathrm{ps}$ after removing the instrument broadening. The ultrafast time-resolved spectroscopic studies were recorded by a femtosecond up-conversion system (FOG100 , CDP) pumped by SHG of the same femtosecond pulse laser. In the experiment, the sample emission generated from a rotating sample cell and the interrogation gate pulse at the designated de-lay time was focused on a BBO crystal with respect to the pump pulse for frequency summation. A $\lambda / 2$ wave plate was used to set polarization at the magic angle of $54.7^{\circ}$ between pump and gate pulse to avoid fluorescence anisotropy. The femtosecond time-resolved data were fitted to the sum of exponential functions convoluted with the IRF, which is fitted to 150 fs determined by Raman scattering signal.

Transient Absorption spectroscopy. The femtosecond UV/vis transient absorption measurements were performed by a pump- probe spectroscopic setup is based on an ExciPro spectrometer (CDP Systems Corp.). A femtosecond Ti:sapphire amplifier (Spitfire Pro, Spectra Physics) was used as the light source. The output of the system consists of pulses of $780 \mathrm{~nm}, 1 \mathrm{~W}, 120 \mathrm{fs}(\mathrm{fwhm}) 1 \mathrm{kHz}$ repetition rate. The laser amplifier output is first split (50\%) into two beams, in which the pump is converted to designated excitation wavelengths by coupling it into a second-harmonic generator (for $390 \mathrm{~nm}$ excitation). The probe is focused on a $1 \mathrm{~mm}$ thick sapphire plate to generate a white light continuum (450-1000 nm). The pump beam is then passed through a computer-controlled optical chopper and focused $(3 \mathrm{~mm})$ on a rotating sample cell. After passing through the sample cell, the white continuum is coupled into a $100 \mu \mathrm{m}$ optical fiber connected to a diode array. Typically, time-resolved absorption spectra were acquired, averaging over 200 excitation pulses at any delay time. The effective time resolution of the ultrafast spectrometer is determined to be about $180 \mathrm{fs}$. 

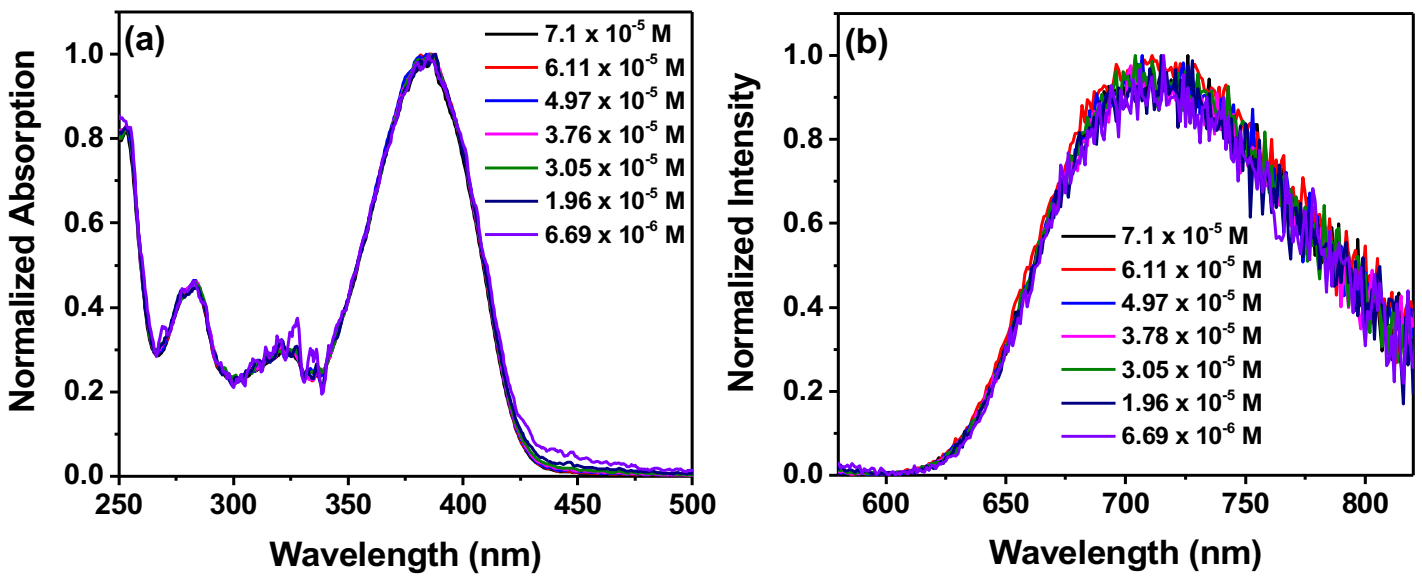

Figure S42. (a) The absorption spectra of $\mathbf{3 N T F}$ in cyclohexane at different concentrations. (b) The emission spectra of $\mathbf{3 N T F}$ in cyclohexane at different concentrations. The results show that the absorption and emission profile is concentration-independent within $6 \times 10^{-6}-10^{-4}$ M. $\lambda_{\text {ex }}=380 \mathrm{~nm}$.

\section{The possibility of the radical emission.}

The thiol-containing molecules often generate thiyl radical upon UV excitation. We have searched relevant literatures on this issue and found that most studies didn't report the bleaching signal caused by the radical emission in transient absorption (TA) measurement..$^{2-5}$ Also, the TA of thiyl radical is the same as the absorption profile of the thiyl radical measured in the steady state. ${ }^{6}$ These observations indicate that the doublet thiyl radicals are generated in the ground state after photodissociation with rather small probability to fluoresce. In other words, if one wants to measure the emission spectrum of thiyl radicals, direct excitation on the thiyl radicals in the ground state is necessary. If this is the case, the excitation spectra should change dramatically compared with the absorption spectrum of the reactant. In our case, the excitation spectra monitored at the observed emission band is identical with the absorption spectrum of $\mathbf{3 N T F}$, discounting the possibility of radical emission (see Figure 4). Moreover, if the observed emission band at $710 \mathrm{~nm}$ is the radical emission, similar emission profile should be observed in 3TF and 3FTF. The fact that we didn't observe any emission signal in that region for other thiol analogues could also rule out the possibility of thiyl radical emission.

In another approach, we performed femtosecond transient absorption (TA) measurement, which is shown in Figure $\mathbf{S 4 3}$. At $\mathrm{t}=0$, an intense, broad 480-600 nm TA signal ca. $560 \mathrm{~nm}$ and a bleaching signal at $710 \mathrm{~nm}$ can be observed. The TA signal exhibits a short decay time constant of 44 ps, followed by a decay with 120 ps (see 
Figure S44). Note that upon monitored at e.g. $2.5 \mathrm{ps}$, the spectral profile maximized at $570 \mathrm{~nm}$ is slightly different that $(550 \mathrm{~nm}$ ) monitored at $45 \mathrm{ps}$ (see Figure $\mathbf{S 4 3 b}$ ). On the other hand, the $710 \mathrm{~nm}$ bleaching signal in TA, which can be attributed to the stimulated emission signal for $\mathbf{3 N T F}$, is gradually hidden by the appearance of a TA signal at $>$ $710 \mathrm{~nm}$ with a rise time constant of $\sim 50 \mathrm{ps}$ (see Figure S44). To rationalize our timeresolved results, i.e., fluorescence up-conversion and TA data, we first assign the 550 nm TA signal to the transient absorption of the excited-state proton-transferred tautomer. This is mainly due to the fact that its 120 ps time constant matches the lifetime of the tautomer emission in the PL measurement. On the other hand, the $570 \mathrm{~nm}$ TA signal with a 44 ps decay time constant, which is in the same magnitude as the rise component (50 ps) of the $>710 \mathrm{~nm}$ TA signal, is tentatively assigned to the absorption of 3NTF radical pair formed in the ground state. In other words, upon excitation of $\mathbf{3 N T F}$, the adiabatic (along the excited state potential energy surface) ESIPT is accompanied with a non-adiabatic process, to a certain extent, forming radical pair in the ground state. The radical pair then dissociates within $44 \mathrm{ps}$, forming the free radical that has absorption band at $>710 \mathrm{~nm}$. To support this viewpoint, Table S1 (see also below) lists three lower lying absorption of thiyl radical obtained by TD-DFT calculation. Among them, the $\mathrm{D}_{0}$ $\rightarrow \mathrm{D}_{2}$ absorption for thiyl radical around $800 \mathrm{~nm}$ is most probably observed in the TA measurement due to the relatively largely oscillator strength than the other two transitions (Table S1 and Figure S45). Accordingly, it is reasonable to assign the $>710$ nm positive TA band to the radical absorption. The well matching between the decay time of $44 \mathrm{ps}$ at $550 \mathrm{~nm}$ and the rise time of $50 \mathrm{ps}$ at $710 \mathrm{~nm}$ can further support the current conjecture. Overall, both TA results and DFT computational results deny the argument that the observed $710 \mathrm{~nm}$ emission originates from the thiyl radical.
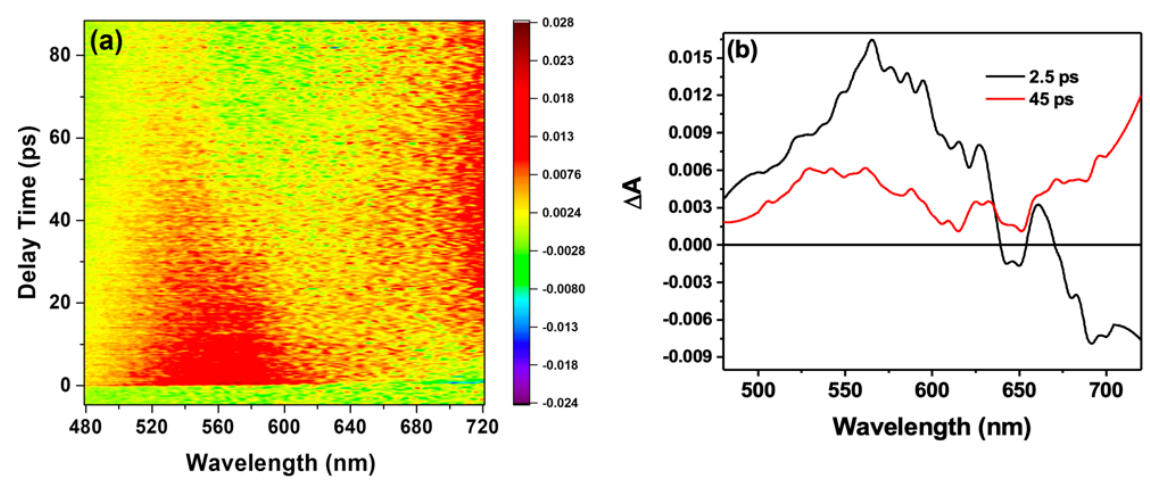

Figure S43. (a) The transient absorption spectrum for 3NTF in cyclohexane at $298 \mathrm{~K}$. (b) The transient spectral profile acquired at $2.5 \mathrm{ps}$ and $45 \mathrm{ps} . \lambda_{\mathrm{ex}}=390 \mathrm{~nm}$. 

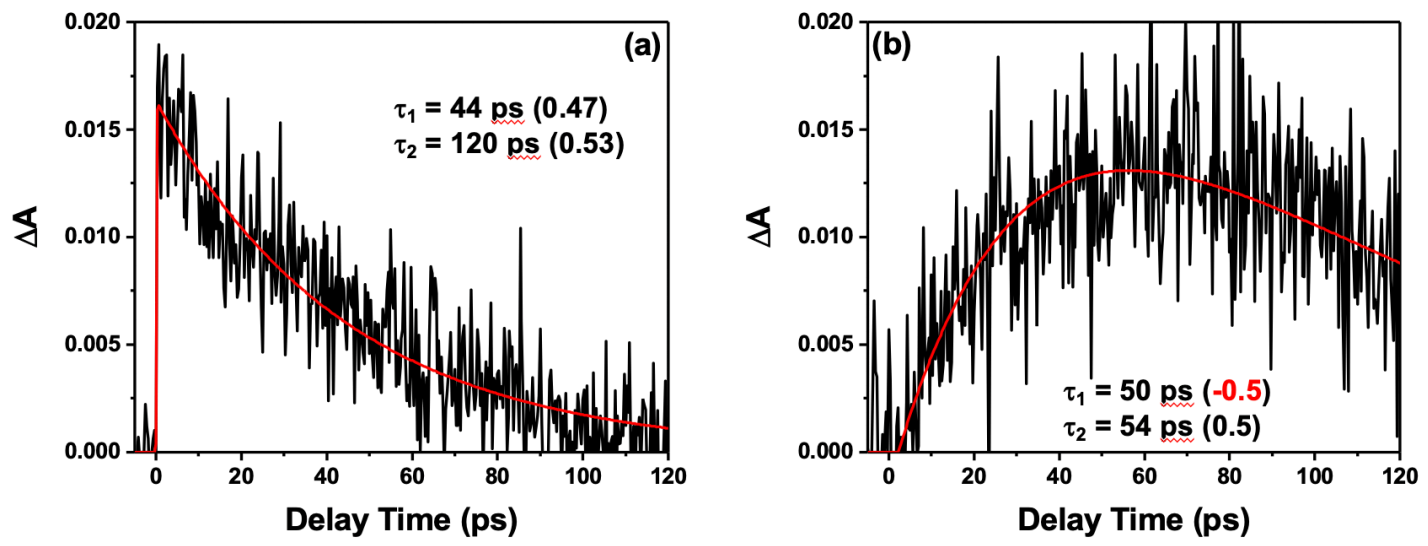

Figure S44. The kinetic traces for the transient absorption signal at (a) $535 \mathrm{~nm}$ and (b) $710 \mathrm{~nm}$ in cyclohexane at $298 \mathrm{~K}$.

Table S1. Calculated excitation energies of the low-lying excited states in the optimized $\mathbf{D}_{\mathbf{0}}$ geometry of deprotonated $\mathbf{3 N T F}$ in the neutral radical form.

\begin{tabular}{|l|l|l|l|}
\hline$@ \mathbf{D}_{\mathbf{0}}$ & Energy $(\mathrm{eV}, \mathrm{nm})$ & contribution & Oscillator strength $(\mathrm{f})$ \\
\hline $\mathrm{D}_{1}\left(\mathrm{n} \pi^{*} / \pi \pi^{*}\right)$ & $(0.54,2297)$ & $84_{\mathrm{B}} \rightarrow 86_{\mathrm{B}}(0.26)$ & $6 \mathrm{E}-4$ \\
& & $85_{\mathrm{B}} \rightarrow 86_{\mathrm{B}}(0.73)$ & \\
\hline $\mathrm{D}_{2}\left(\pi \pi^{*} / \mathrm{n} \pi^{*}\right)$ & $(1.54,804)$ & $84_{\mathrm{B}} \rightarrow 86_{\mathrm{B}}(0.71)$ & 0.11 \\
& & $85_{\mathrm{B}} \rightarrow 86_{\mathrm{B}}(0.25)$ & \\
\hline $\mathrm{D}_{3}\left(\mathrm{n} \pi^{*}\right)$ & $(2.22,557)$ & $83_{\mathrm{B}} \rightarrow 86_{\mathrm{B}}(0.91)$ & $1 \mathrm{E}-3$ \\
\hline
\end{tabular}

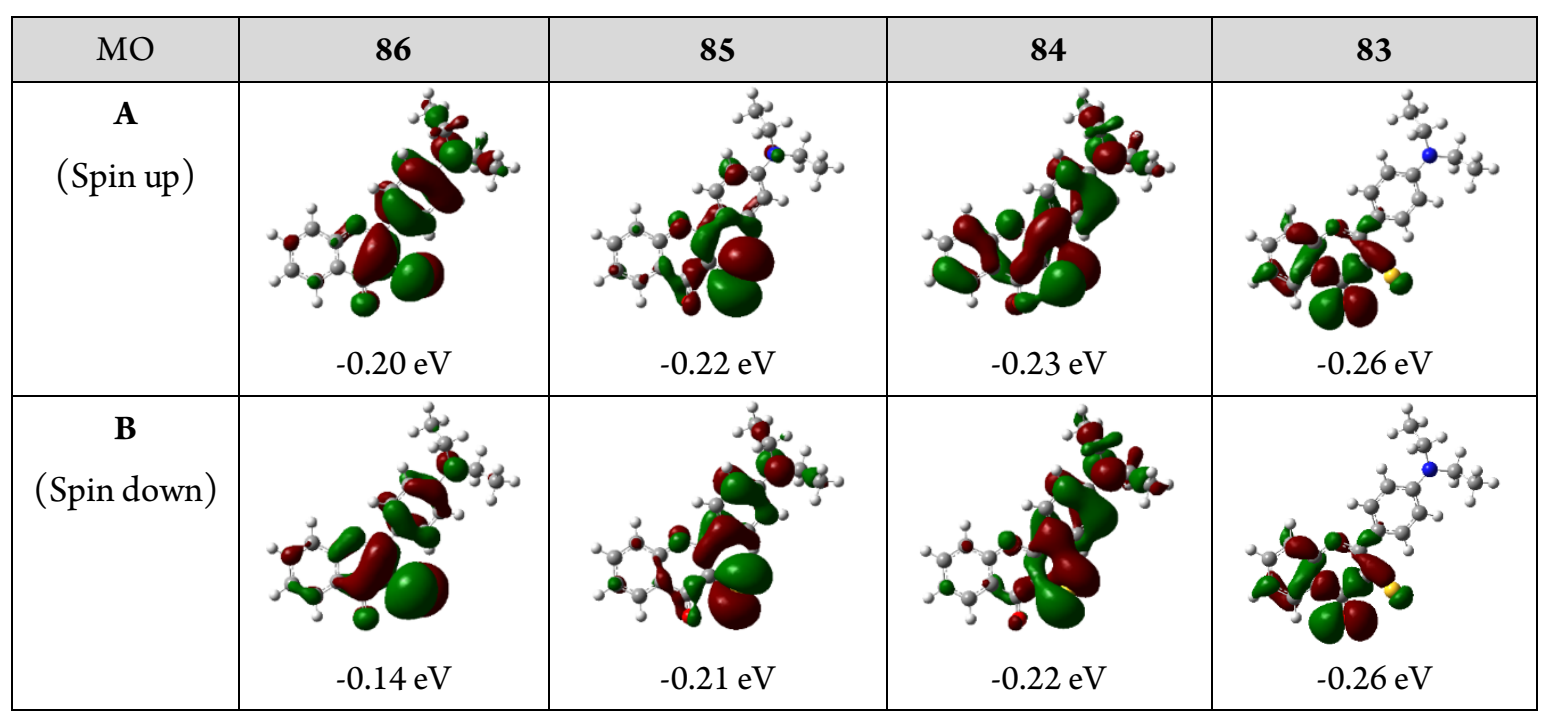

Figure S45. Calculated molecular orbitals in the optimized $\mathrm{D}_{0}$ geometry of deprotonated 3NTF in the neutral radical form at the UB3LYP/6-311++G(3df,3pd) level. 


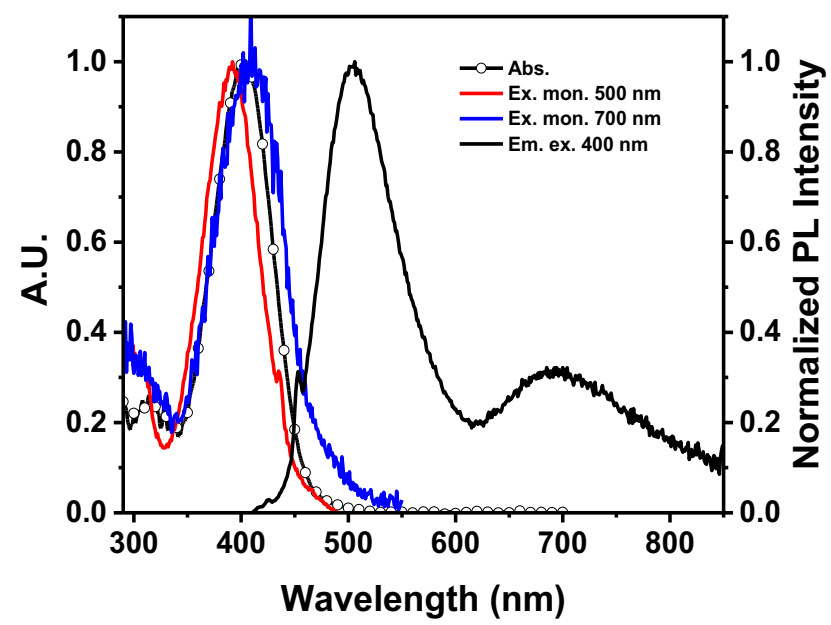

Figure S46. The absorption (circle and line) and emission (solid line) spectra for 3NTF in ethanol at $298 \mathrm{~K} . \lambda_{\mathrm{ex}}=390 \mathrm{~nm}$.
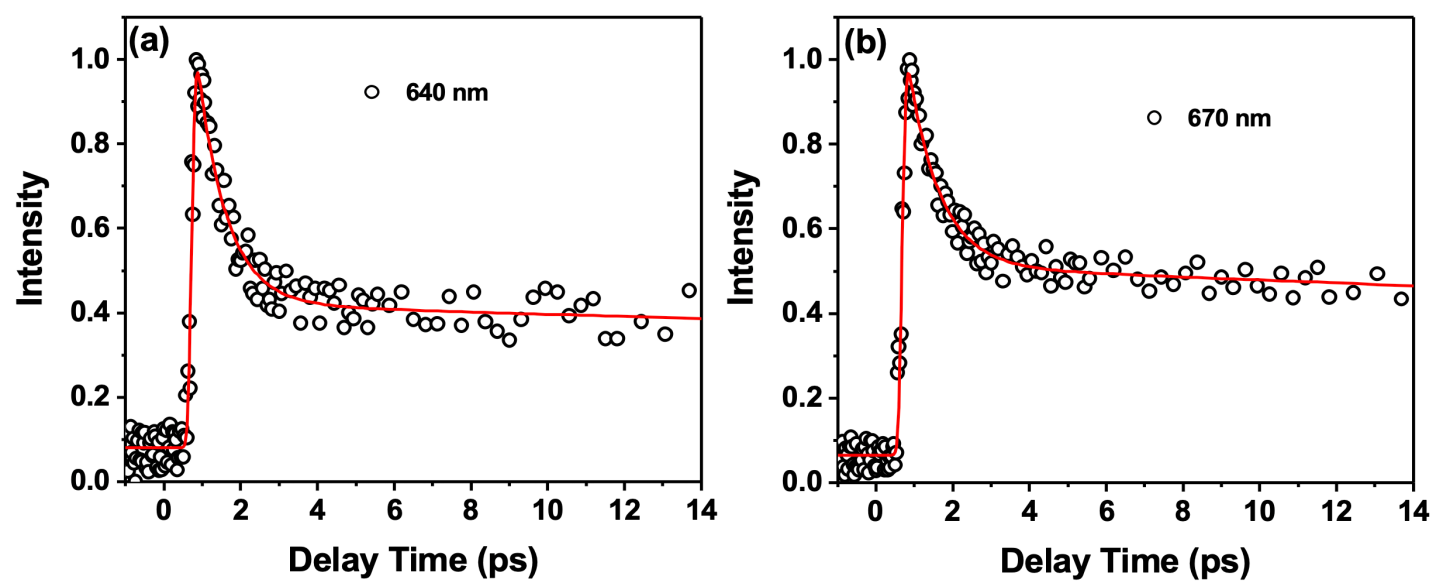

Figure S47. The kinetic trace of 3NTF at (a) $640 \mathrm{~nm}$ and (b) $670 \mathrm{~nm}$ acquired by fluorescence up-conversion technique in cyclohexane at $298 \mathrm{~K}$. $\lambda_{\mathrm{ex}}=390 \mathrm{~nm}$.

Table S2. The time-resolved data for 3NTF acquired by fluorescence up-conversion measurement in cyclohexane at $298 \mathrm{~K}$.

\begin{tabular}{cc}
\hline$\lambda_{\text {mon. }}(\mathbf{n m})$ & $\tau_{\text {obs. }}(\mathbf{p s})$ \\
\hline $\mathbf{4 7 0}$ & $<0.18^{\mathrm{a}}$ \\
$\mathbf{6 4 0}$ & $0.75(0.68), 120(0.32)^{\mathrm{b}}$ \\
$\mathbf{6 7 0}$ & $0.90(0.58), 120(0.42)^{\mathrm{b}}$ \\
$\mathbf{7 0 0}$ & $1.58(0.56), 120(0.44)^{\mathrm{b}}$ \\
\hline
\end{tabular}

${ }^{a}$ this value is beyond the instrument limit. ${ }^{b}$ the value obtained by TCSPC was applied to the data fitting. 

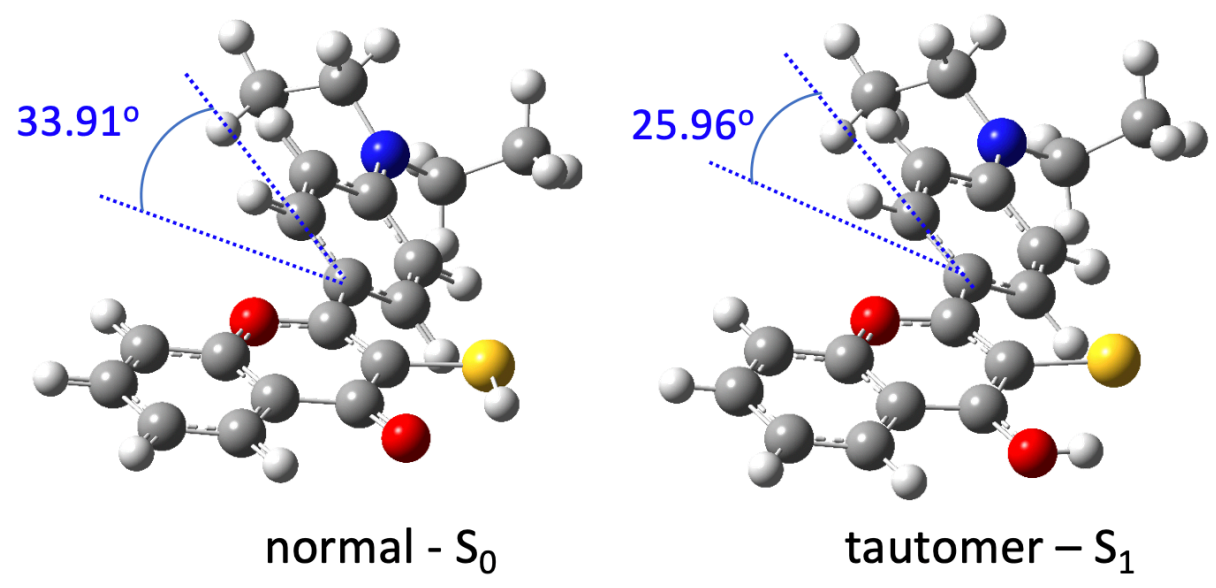

Figure S48. The calculated geometries for $\mathbf{3 N T F}$ at $\mathrm{S}_{1}$ and $\mathrm{S}_{1}$ ' states. The angle represents the dihedral angle between phenyl group and chromenone moiety.
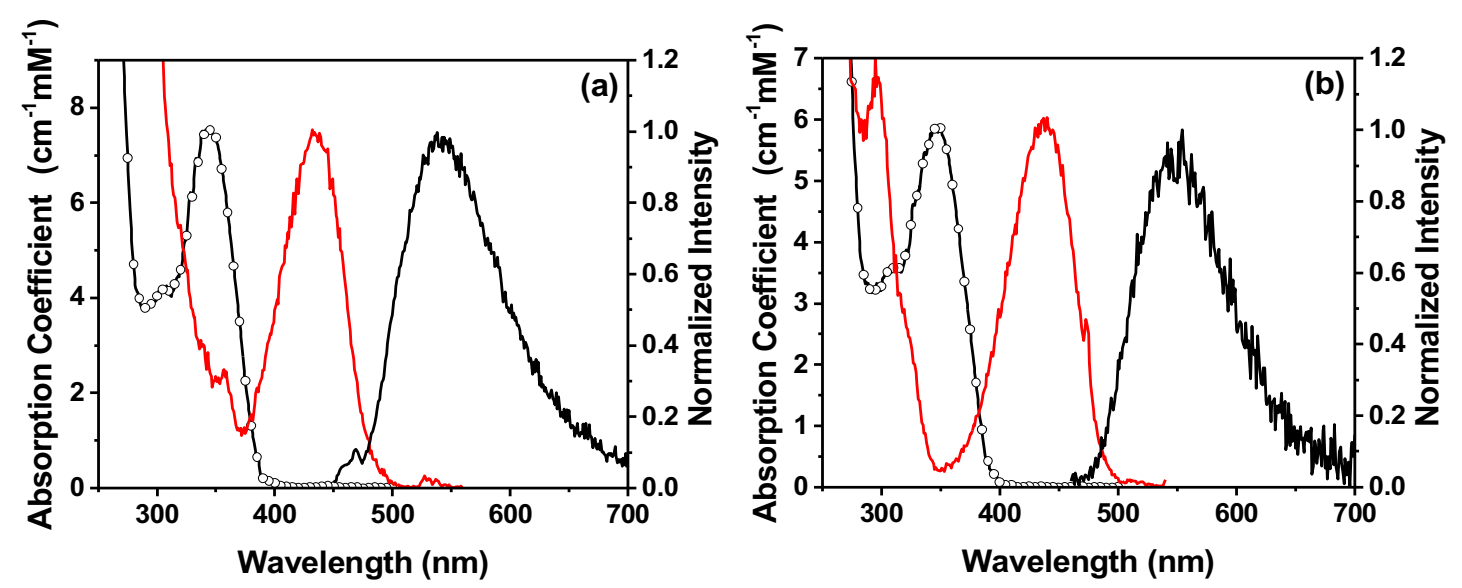

Figure S49. (a) The absorption (circle and line) and emission (solid line) spectra of 3TF in cyclohexane at $298 \mathrm{~K}$. The excitation spectrum of $\mathbf{3 T F}$ monitored at $540 \mathrm{~nm}$ is shown in red line. (b) The absorption (circle and line) and emission (solid line) spectra of 3FTF in cyclohexane at 298K. The excitation spectrum of 3FTF monitored at 550 $\mathrm{nm}$ is shown in red line. Note that the excitation spectrum appearing in 400-500 nm region does not contribute to any absorption, indicating its very trace population, which has been identified as the anion species (see Figure S50). 


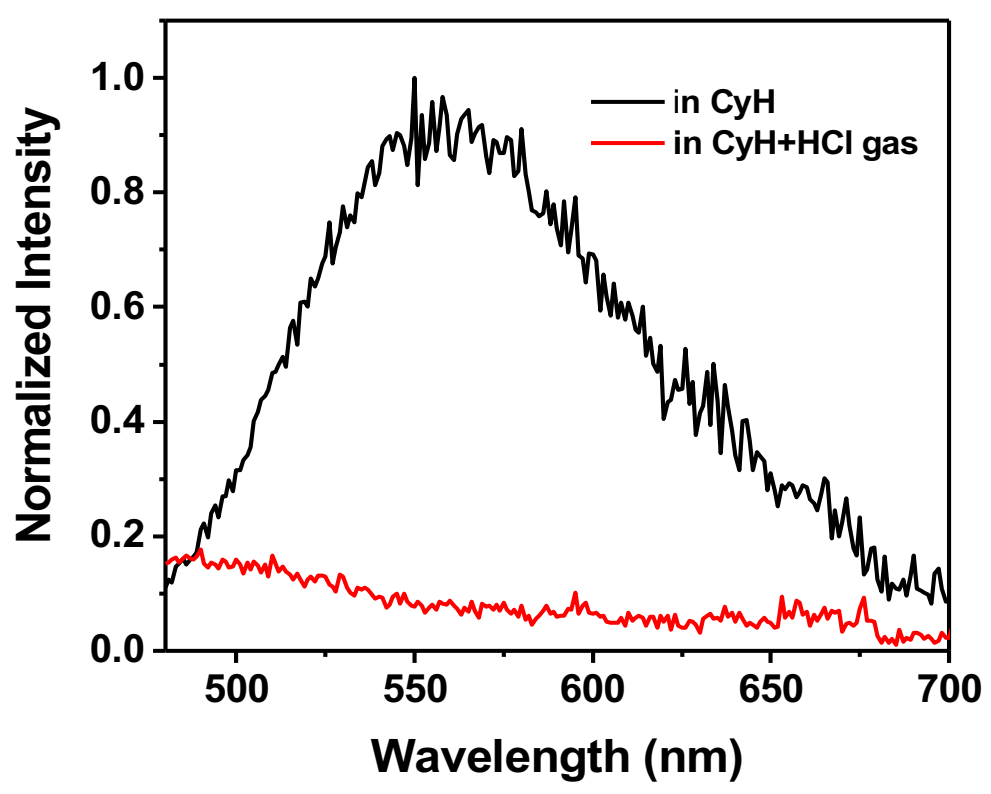

Figure S50. The emission spectra of 3FTF in cyclohexane and cyclohexane $+\mathrm{HCl}$ gas at $298 \mathrm{~K} . \lambda_{\mathrm{ex}}=410 \mathrm{~nm}$

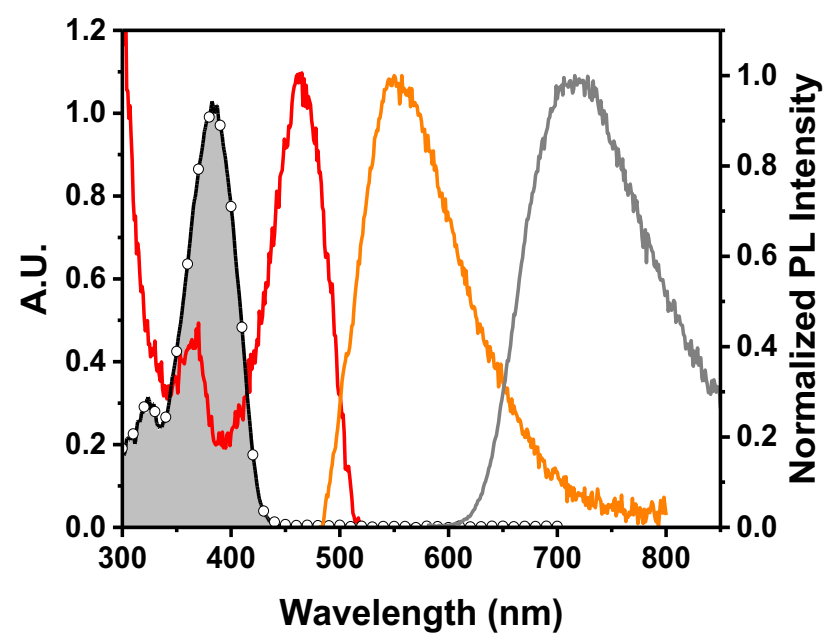

Figure S51. The absorption (grey area) and emission (grey solid line) spectra of 3NTF in cyclohexane at $298 \mathrm{~K}\left(\lambda_{\mathrm{ex}}=390 \mathrm{~m}\right.$, ) Upon adding a trace of triethylamine into the cyclohexane, also shown are the absorption (black circle and line) spectrum of 3NTF. Note that the absorption (trimethylamine added) profile is nearly the same as that in pure cyclohexane with no absorption at $>450 \mathrm{~nm}$. However, upon $470 \mathrm{~nm}$ excitation a new emission band (see orange solid line) appears maximized at $550 \mathrm{~nm}$. The excitation spectrum monitored at $550 \mathrm{~nm}$ shown a maximum at $470 \mathrm{~nm}$ (see the red solid line). The results indicate trace of ion-pair formation in the basic solution, that gives rise to the ion-pair $550 \mathrm{~nm}$ emission in nonpolar cyclohexane. 
Table S3. Calculated excitation energies of the low-lying excited states in the optimized $\mathbf{S}_{\mathbf{1}}$ geometry of deprotonated $\mathbf{3 N T F}$ with the $\mathrm{Na}^{+}$counterion as the ion-pair form.

\begin{tabular}{|l|l|l|l|}
\hline$@ \mathbf{S}_{1}$ & Energy $(\mathrm{eV}, \mathrm{nm})$ & contribution & Oscillator strength (f) \\
\hline $\mathrm{S}_{1}\left(\pi \pi^{*}\right)$ & $(2.08,597)$ & $\mathrm{H} \rightarrow \mathrm{L}(0.97)$ & 0.23 \\
\hline $\mathrm{S}_{2}\left(\mathrm{n} \pi^{*}\right)$ & $(2.46,504)$ & $\mathrm{H}-1 \rightarrow \mathrm{L}(0.97)$ & $5 \mathrm{E}-3$ \\
\hline
\end{tabular}

\begin{tabular}{|c|c|c|c|}
\hline MO & L & H & H-1 \\
\hline & & \\
\hline
\end{tabular}

Figure S52. Calculated molecular orbitals in the optimized $\mathrm{S}_{1}$ geometry of deprotonated 3NTF with the $\mathrm{Na}^{+}$counterion as the ion-pair form at the B3LYP/6$311++\mathrm{G}(3 \mathrm{df}, 3 \mathrm{pd})$ level.

Table S4. Calculated excitation energies of the low-lying excited states in the optimized $S_{1}$ geometry of deprotonated $\mathbf{3 N T F}$ in the anion form.

\begin{tabular}{|l|l|l|l|}
\hline$@ \mathbf{S}_{\mathbf{1}}$ & Energy $(\mathrm{eV}, \mathrm{nm})$ & contribution & Oscillator strength $(\mathrm{f})$ \\
\hline $\mathrm{S}_{1}\left(\mathrm{n} \pi^{*}\right)$ & $(1.32,936)$ & $\mathrm{H} \rightarrow \mathrm{L}(0.98)$ & $2 \mathrm{E}-4$ \\
\hline $\mathrm{S}_{2}\left(\pi \pi^{*}\right)$ & $(1.81,685)$ & $\mathrm{H}-1 \rightarrow \mathrm{L}(0.99)$ & 0.15 \\
\hline
\end{tabular}

\begin{tabular}{|c|c|c|c|}
\hline MO & L & H \\
\hline & & \\
\hline
\end{tabular}

Figure S53. Calculated molecular orbitals in the optimized $\mathrm{S}_{1}$ geometry of deprotonated 3NTF in the anion form at the B3LYP/6-311++G(3df,3pd) level. 


\section{Computational Results}

\section{Computational Method}

Density functional theory (DFT) and time-dependent density functional theory (TDDFT) $)^{7-11}$ calculations of all compounds were carried out using the hybrid exchangecorrelation functional B3LYP ${ }^{12-13}$ with the $6-311++\mathrm{G}(3 \mathrm{df}, 3 \mathrm{pd})$ basis set $^{14}$ in Gaussian $^{2}$ 16. ${ }^{15}$ Geometry optimizations were performed for the singlet ground state, first singlet excited state, and the first triplet state. Solvation corrections for the solvent used cyclohexane $(\varepsilon=2.0165)$ and were calculated using the polarizable continuum model $(\mathrm{PCM})^{16}$ with the default UFF radii. TDDFT calculations based on the optimized ground-state and/or excited-state structures were performed.

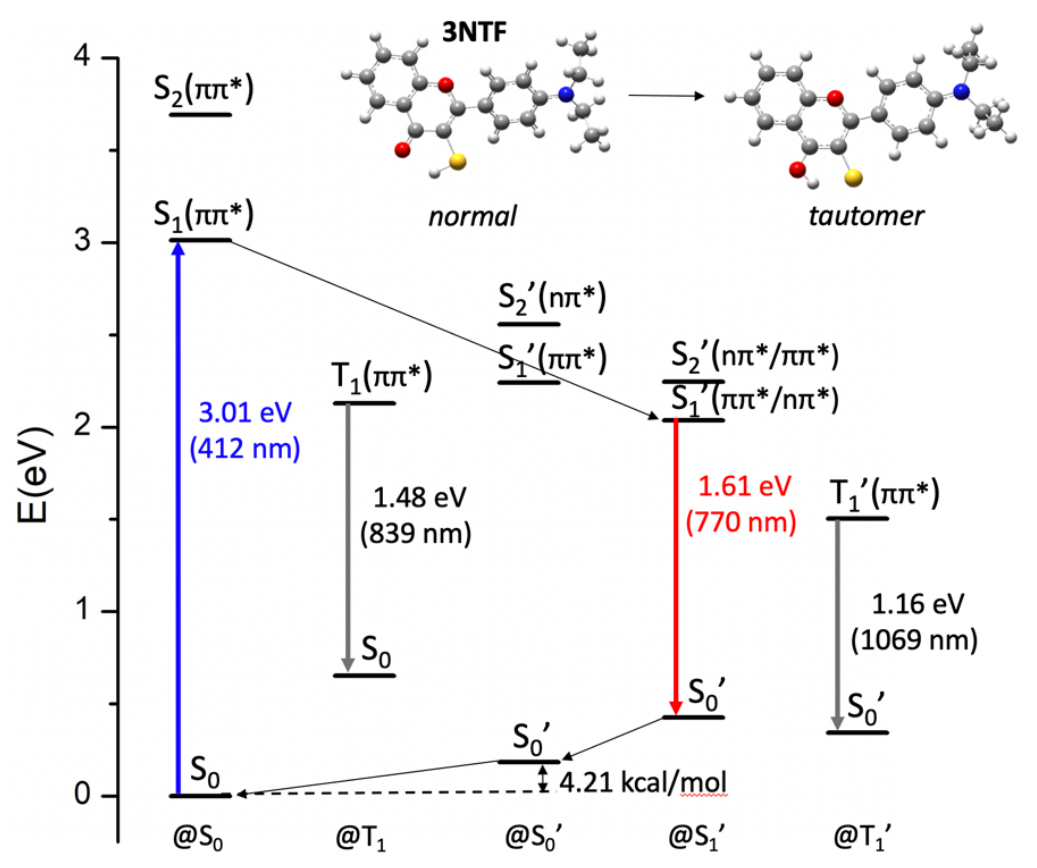

Figure S54. The calculated various energy levels for $\mathbf{3 N T F}$ in the optimized $\mathrm{S}_{0}, \mathrm{~S}_{1}$ and $\mathrm{T}_{1}$ geometries at the normal form and the proton-transfer tautomer form (for which the states are denoted with a prime sign). 


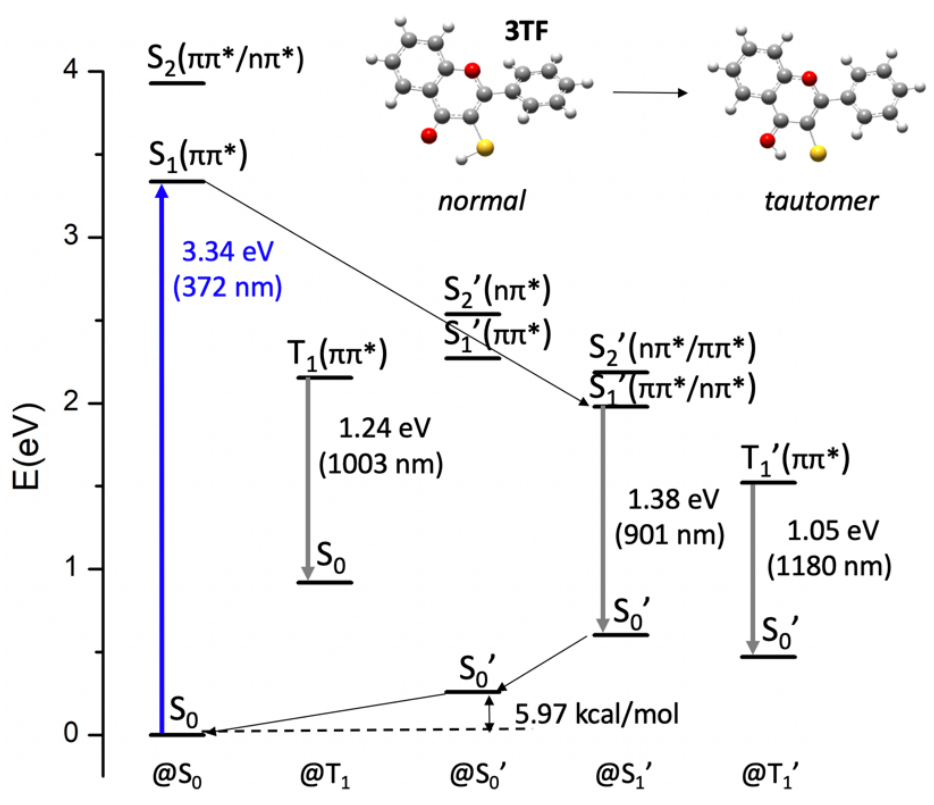

Figure S55. The calculated various energy levels for $\mathbf{3 T F}$ in the optimized $\mathrm{S}_{0}, \mathrm{~S}_{1}$ and $\mathrm{T}_{1}$ geometries at the normal form and the proton-transfer tautomer form (for which the states are denoted with a prime sign).

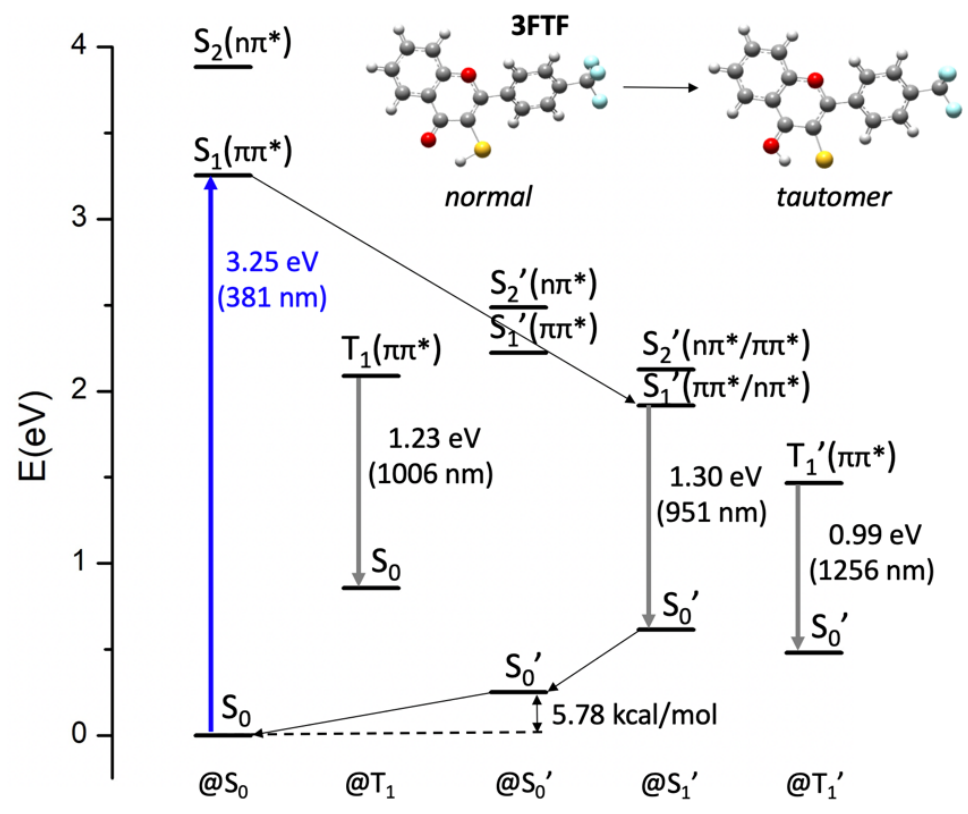

Figure S56. The calculated various energy levels for $\mathbf{3 F T F}$ in the optimized $\mathrm{S}_{0}, \mathrm{~S}_{1}$ and $\mathrm{T}_{1}$ geometries at the normal form and the proton-transfer tautomer form (for which the states are denoted with a prime sign). 


\begin{tabular}{|c|c|c|c|c|}
\hline MO & HОMO-2 & HOMO-1 & НОМо & LUMO \\
\hline 3TF & - $0.27 \mathrm{eV}$ & $-0.26 \mathrm{eV}$ & $-0.22 \mathrm{eV}$ & $-0.08 \mathrm{eV}$ \\
\hline 3TF' & $-0.27 \mathrm{eV}$ & $0.22 \mathrm{eV}$ & $-0.20 \mathrm{eV}$ & $-0.11 \mathrm{eV}$ \\
\hline 3FTF & $-0.28 \mathrm{eV}$ & $-0.27 \mathrm{eV}$ & $-0.23 \mathrm{eV}$ & $-0.09 \mathrm{eV}$ \\
\hline 3FTF' & $-0.28 \mathrm{eV}$ & $-0.23 \mathrm{eV}$ & $-0.21 \mathrm{eV}$ & $-0.12 \mathrm{eV}$ \\
\hline 3NTF & $-0.26 \mathrm{eV}$ & $-0.23 \mathrm{eV}$ & $-0.20 \mathrm{eV}$ & $-0.07 \mathrm{eV}$ \\
\hline 3NTF' & $-0.22 \mathrm{eV}$ & $-0.21 \mathrm{eV}$ & $-0.19 \mathrm{eV}$ & $-0.09 \mathrm{eV}$ \\
\hline 3MeNTF & $-0.25 \mathrm{eV}$ & $-0.22 \mathrm{eV}$ & $-0.20 \mathrm{eV}$ & $\begin{array}{l}0.07 \mathrm{eV} \\
-0.07\end{array}$ \\
\hline
\end{tabular}

Figure S57. Calculated molecular orbitals in the optimized ground-state geometry of the normal or the tautomer forms (denoted by prime) of various compounds at the 
B3LYP/6-311++G(3df,3pd) level.

Table S5. Theoretical absorption and luminescence data for different systems in the normal and tautomer (denoted by prime) forms in cyclohexane.

\begin{tabular}{|l|l|l|l|l|l|}
\hline & $\begin{array}{l}\mathrm{E}\left(\mathrm{S}_{1}\right) @ \mathrm{~S}_{0} \\
(\mathrm{eV}, \mathrm{nm})\end{array}$ & $\begin{array}{l}\text { Oscillator } \\
\text { strength }(\mathrm{f})\end{array}$ & $\begin{array}{l}\mathrm{E}\left(\mathrm{S}_{1}\right) @ \mathrm{~S}_{1} \\
(\mathrm{eV}, \mathrm{nm})\end{array}$ & $\begin{array}{l}\text { Oscillator } \\
\text { strength }(\mathrm{f})\end{array}$ & $\begin{array}{l}{\left[\mathrm{E}\left(\mathrm{T}_{1}\right)\right] @ \mathrm{~T}_{1}} \\
(\mathrm{eV}, \mathrm{nm})\end{array}$ \\
\hline 3TF & $(3.34,372)$ & 0.20 & $(1.38,901)$ & 0.04 & $(1.24,1003)$ \\
\hline 3TF' & $(2.01,617)$ & 0.12 & --- & --- & $(1.05,1180)$ \\
\hline 3FTF & $(3.25,381)$ & 0.20 & $(2.66,466)$ & 0.24 & $(1.23,1006)$ \\
\hline 3FTF' & $(1.97,628)$ & 0.13 & $(1.30,951)$ & 0.04 & $(0.99,1256)$ \\
\hline 3NTF & $(3.01,412)$ & 0.60 & --- & --- & $(1.48,839)$ \\
\hline 3NTF' & $(2.06,603)$ & 0.32 & $(1.61,770)$ & 0.13 & $(1.16,1069)$ \\
\hline 3MeNTF & $(3.18,390)$ & 0.53 & $(2.53,490)$ & 0.36 & $(0.94,1326)$ \\
\hline
\end{tabular}

Table S6. Calculated excitation energies of the low-lying excited states in the optimized S' 1 geometry of $\mathbf{3 N T F}$ (in the tautomer form).

\begin{tabular}{|l|l|l|}
\hline$@ \mathbf{S}^{\prime}{ }_{1}$ & Energy $(\mathrm{eV})$ & contribution \\
\hline $\mathrm{T}^{\prime}{ }_{1}\left(\pi \pi^{*}\right)$ & 0.99 & $\mathrm{H} \rightarrow \mathrm{L}(97 \%)$ \\
\hline $\mathrm{S}^{\prime}{ }_{1}\left(\pi \pi^{*} / \mathrm{n} \pi^{*}\right)$ & 1.61 & $\begin{array}{l}\mathrm{H} \rightarrow \mathrm{L}(91 \%) \\
\mathrm{H}-1 \rightarrow \mathrm{L}(7 \%)\end{array}$ \\
\hline $\mathrm{T}^{\prime}{ }_{2}\left(\mathrm{n} \pi^{*}\right)$ & 1.74 & $\mathrm{H}-1 \rightarrow \mathrm{L}(98 \%)$ \\
\hline $\mathrm{S}^{\prime}{ }_{2}\left(\mathrm{n} \pi^{*} / \pi \pi^{*}\right)$ & 1.82 & $\begin{array}{l}\mathrm{H} \rightarrow \mathrm{L} \mathrm{(7 \% )} \\
\mathrm{H}-1 \rightarrow \mathrm{L}(93 \%)\end{array}$ \\
\hline $\mathrm{T}^{\prime}{ }_{3}\left(\pi \pi^{*}\right)$ & 2.17 & $\mathrm{H}-2 \rightarrow \mathrm{L}(96 \%)$ \\
\hline
\end{tabular}

Table S7. Calculated excitation energies and the corresponding molecular orbital transitions of the low-lying excited states in the optimized $\mathbf{S}^{\prime}{ }_{1}$ geometry of $\mathbf{3 T F}$ (in the tautomer form).

\begin{tabular}{|l|l|l|}
\hline a $\mathbf{S}^{\prime}{ }_{1}$ & Energy $(\mathrm{eV})$ & contribution \\
\hline $\mathrm{T}^{\prime}{ }_{1}\left(\pi \pi^{*}\right)$ & 0.82 & $\mathrm{H} \rightarrow \mathrm{L}(99 \%)$ \\
\hline $\mathrm{S}^{\prime}{ }_{1}\left(\pi \pi^{*} / \mathrm{n} \pi^{*}\right)$ & 1.38 & $\begin{array}{l}\mathrm{H} \rightarrow \mathrm{L}(82 \%) \\
\mathrm{H}-1 \rightarrow \mathrm{L}(17 \%)\end{array}$ \\
\hline $\mathrm{T}^{\prime}{ }_{2}\left(\mathrm{n} \pi^{*}\right)$ & 1.45 & $\mathrm{H}-1 \rightarrow \mathrm{L} \mathrm{(97 \% )}$ \\
\hline $\mathrm{S}^{\prime}{ }_{2}\left(\mathrm{n} \pi^{*} / \pi \pi^{*}\right)$ & 1.58 & $\begin{array}{l}\mathrm{H} \rightarrow \mathrm{L}(17 \%) \\
\mathrm{H}-1 \rightarrow \mathrm{L}(82 \%)\end{array}$ \\
\hline $\mathrm{T}^{\prime}{ }_{3}\left(\pi \pi^{*}\right)$ & 2.58 & $\mathrm{H} \rightarrow \mathrm{L}+1(88 \%)$ \\
\hline
\end{tabular}


Table S8. Calculated excitation energies of the low-lying excited states in the optimized $\mathbf{S}_{1}$ geometry of $\mathbf{3 F T F}$ (in the tautomer form).

\begin{tabular}{|l|l|l|}
\hline$@ \mathbf{S}^{\prime}{ }_{1}$ & Energy $(\mathrm{eV})$ & contribution \\
\hline $\mathrm{T}^{\prime}{ }_{1}\left(\pi \pi^{*}\right)$ & 0.76 & $\mathrm{H} \rightarrow \mathrm{L}(99 \%)$ \\
\hline $\mathrm{S}^{\prime}{ }_{1}\left(\pi \pi^{*} / \mathrm{n} \pi^{*}\right)$ & 1.30 & $\begin{array}{l}\mathrm{H} \rightarrow \mathrm{L}(81 \%) \\
\mathrm{H}-1 \rightarrow \mathrm{L}(19 \%)\end{array}$ \\
\hline $\mathrm{T}^{\prime}{ }_{2}\left(\mathrm{n} \pi^{*}\right)$ & 1.38 & $\mathrm{H}-1 \rightarrow \mathrm{L} \mathrm{(97 \% )}$ \\
\hline $\mathrm{S}^{\prime}{ }_{2}\left(\mathrm{n} \pi^{*} / \pi \pi^{*}\right)$ & 1.51 & $\begin{array}{l}\mathrm{H} \rightarrow \mathrm{L}(19 \%) \\
\mathrm{H}-1 \rightarrow \mathrm{L}(81 \%)\end{array}$ \\
\hline $\mathrm{T}^{\prime}{ }_{3}\left(\pi \pi^{*}\right)$ & 2.50 & $\mathrm{H} \rightarrow \mathrm{L}+1(89 \%)$ \\
\hline
\end{tabular}

\section{Reference}

1. Wang, X.; Liu, J.; Zhang, Y., An Efficient One-Pot Synthesis and Anticancer Activity of 4'-Substituted Flavonoids. Russ. J. Gen. Chem. 2018, 88, 1036-1041.

2. Ito, O., Flash photolysis study on reversible addition reactions of thiyl radicals. Res. Chem. Intermed. 1995, 21, 69-93.

3. Ishizaka, S.-i.; Kotani, M., Estimation of extinction coefficient and radiative lifetime of a free radical, p-aminophenylthiyl. Chem. Phys. Lett. 1987, 139, 89-92.

4. Bhattacharyya, K.; Das, P. K.; Ramamurthy, V.; Rao, V. P., Triplet-state photophysics and transient photochemistry of cyclic enethiones. A laser flash photolysis study. J. Chem. Soc., Faraday Trans. 2 1986, 82, 135-147.

5. Morine, G.; Kuntz, R., Spectral shifts of the p-aminophenylthyl radical absorption and emission in solution. Chem. Phys. Lett. 1979, 67, 552-554.

6. Lembke, R. R.; Natarajan, L. V.; Kuntz, R. R., The extinction coefficient for the p-aminophenylthiyl radical as determined by reaction with galvinoxyl. J. Photochem. 1983, 21, 157-166.

7. Stratmann, R. E.; Scuseria, G. E.; Frisch, M. J., An efficient implementation of time-dependent density-functional theory for the calculation of excitation energies of large molecules. J. Chem. Phys. 1998, 109, 8218-8224.

8. Casida, M. E.; Jamorski, C.; Casida, K. C.; Salahub, D. R., Molecular excitation energies to high-lying bound states from time-dependent density-functional response theory: Characterization and correction of the time-dependent local density approximation ionization threshold. J. Chem. Phys. 1998, 108, 4439-4449.

9. Bauernschmitt, R.; Ahlrichs, R.; Hennrich, F. H.; Kappes, M. M., Experiment versus time dependent density functional theory prediction of fullerene electronic absorption. J. Am. Chem. Soc. 1998, 120, 5052-5059.

10. Petersilka, M.; Gossmann, U. J.; Gross, E. K. U., Excitation energies from time- 
dependent density-functional theory. Phys. Rev. Lett. 1996, 76, 1212-1215.

11. Jamorski, C.; Casida, M. E.; Salahub, D. R., Dynamic polarizabilities and excitation spectra from a molecular implementation of time-dependent densityfunctional response theory: N-2 as a case study. J. Chem. Phys. 1996, 104, 5134-5147. 12. Stephens, P. J.; Devlin, F. J.; Chabalowski, C. F.; Frisch, M. J., Ab Initio Calculation of Vibrational Absorption and Circular Dichroism Spectra Using Density Functional Force Fields. J. Phys. Chem. 1994, 98, 11623-11627.

13. Becke, A. D., Density-Functional Thermochemistry .3. The Role of Exact Exchange. J. Chem. Phys. 1993, 98, 5648-5652.

14. Frisch, M. J.; Pople, J. A.; Binkley, J. S., Self-Consistent Molecular-Orbital Methods .25. Supplementary Functions for Gaussian-Basis Sets. J. Chem. Phys. 1984, 80, 3265-3269.

15. Frisch, M. J.; Trucks, G. W.; Schlegel, H. B.; Scuseria, G. E.; Robb, M. A.;

Cheeseman, J. R.; Scalmani, G.; Barone, V.; Petersson, G. A.; Nakatsuji, H.; Li, X.; Caricato, M.; Marenich, A. V.; Bloino, J.; Janesko, B. G.; Gomperts, R.; Mennucci, B.; Hratchian, H. P.; Ortiz, J. V.; Izmaylov, A. F.; Sonnenberg, J. L.; Williams; Ding, F.; Lipparini, F.; Egidi, F.; Goings, J.; Peng, B.; Petrone, A.; Henderson, T.; Ranasinghe, D.; Zakrzewski, V. G.; Gao, J.; Rega, N.; Zheng, G.; Liang, W.; Hada, M.; Ehara, M.; Toyota, K.; Fukuda, R.; Hasegawa, J.; Ishida, M.; Nakajima, T.; Honda, Y.; Kitao, O.; Nakai, H.; Vreven, T.; Throssell, K.; Montgomery Jr., J. A.; Peralta, J. E.; Ogliaro, F.; Bearpark, M. J.; Heyd, J. J.; Brothers, E. N.; Kudin, K. N.; Staroverov, V. N.; Keith, T. A.; Kobayashi, R.; Normand, J.; Raghavachari, K.; Rendell, A. P.; Burant, J. C.; Iyengar, S. S.; Tomasi, J.; Cossi, M.; Millam, J. M.; Klene, M.; Adamo, C.; Cammi, R.; Ochterski, J. W.; Martin, R. L.; Morokuma, K.; Farkas, O.; Foresman, J. B.; Fox, D. J. Gaussian 16 Rev. A.03, Wallingford, CT, 2016. 16. Tomasi, J.; Persico, M., Molecular-Interactions in Solution - an Overview of Methods Based on Continuous Distributions of the Solvent. Chem. Rev. 1994, 94 , 2027-2094. 